\title{
Palladium-Catalyzed Norbornene-Mediated Tandem Amination/Cyanation Reaction: A Method for the Synthesis of ortho-Aminated Benzonitriles
}

\author{
Bo Luo ${ }^{\dagger, \ddagger}$, Jin-Ming Gao*, and Mark Lautens ${ }^{\star, \ddagger}$ \\ ${ }^{\dagger}$ Shaanxi Key Laboratory of Natural Products \& Chemical Biology, College of Science, Northwest A\&F \\ University, Yangling, Shaanxi, 712100, China \\ ${ }^{\ddagger}$ Department of Chemistry, University of Toronto, 80 St. George Street, Toronto, Ontario, M5S 3H6, Canada . \\ E-mail:jinminggao@nwsuaf.edu.cn \\ mlautens@chem.utoronto.ca
}

Table of contents

General Practical Considerations

Materials S2

Instrumentation S2

Optimization of the Reaction Conditions S3

Detailed Experimental Procedures

Synthesis of O-Benzoyl Hydroxylamines

Synthesis of Synthesis of Aryl Iodides

General Procedure for the Sequential Amination/Cyanation Reaction between Aryl Iodides 1, $N$-benzoyloxyamine 2, and $\mathrm{Zn}(\mathrm{CN})_{2}$.

General Procedure for the Sequential Amination/Cyanation Reaction between Aryl Iodides 4, $N$-benzoyloxyamine $\mathbf{2 a}$, and $\mathrm{Zn}(\mathrm{CN})_{2}$. 


\section{General Practical Considerations}

Unless otherwise stated, all reactions were performed in dry solvents under an inert atmosphere in oven-dried $\left(120^{\circ} \mathrm{C}\right)$ flasks equipped with a magnetic stir bar. Dry solvents were transferred via syringe. Organic solutions were concentrated by rotary evaporation at $25-40{ }^{\circ} \mathrm{C}$ under reduced pressure. Analytical thin layer chromatography (TLC) was performed on pre-coated EMD Silica gel 60 F254 plates $(0.2 \mathrm{~mm}, 60 \AA$ pore size). Visualization was done with $254 \mathrm{~nm}$ UV light source and generally by immersion in potassium permanganate $\left(\mathrm{KMnO}_{4}\right)$, subsequently followed by heating with a heat gun. Flash chromatography was performed with Silicycle ${ }^{\mathrm{TM}}$ Ultra-Pure 230-400 mesh silica gel.

\section{Materials}

All reagents were purchased from commercial sources and used as received: catalysts and ligands were purchased from Strem or Sigma Aldrich. All starting materials were obtained from Aldrich, Combi Blocks and VWR. Tetrahydrofuran and dioxane were distilled over $\mathrm{Na} /$ benzophenone prior to use. Toluene was distilled over $\mathrm{CaH}_{2}$ prior to use.

\section{Instrumentation}

Unless otherwise stated, proton nuclear magnetic resonance spectra ('H NMR) and carbon nuclear magnetic resonance spectra $\left({ }^{13} \mathrm{C}\right.$ NMR) were recorded at $23{ }^{\circ} \mathrm{C}$ with a Bruker Avance III $400 \mathrm{MHz}$, Varian Mercury $400 \mathrm{MHz}$, Varian Mercury $300 \mathrm{MHz}$, or Agilent DDR 500 spectrometer. Chemical shifts are reported in parts per million ( $\mathrm{ppm}$ ) and referenced to the residual protium relative to TMS ( $\mathrm{CDCl}_{3}$ : $\delta 7.26$ for ${ }^{1} \mathrm{H}$ NMR and $\delta 77.16$ for ${ }^{13} \mathrm{C}$ NMR). Spectral data are represented as follows: chemical shift $(\delta)$, multiplicity $(\mathrm{s}=$ singlet, $\mathrm{d}=$ doublet, $\mathrm{t}=$ triplet, $\mathrm{q}=$ quartet, $\mathrm{qn}=$ quintuplet, $\mathrm{sx}=$ sextet, $\mathrm{dd}=$ doublet of doublets, $\mathrm{m}=$ multiplet, $\mathrm{br}=$ broad $)$, coupling constant $(\mathrm{J}, \mathrm{Hz})$, integration. Infrared (IR) spectra were obtained using a Shimadzu FTIR 8400 S spectrometry as a film on a $\mathrm{NaCl}$ plate or a Perkin-Elmer Spectrum 100 instrument equipped with a single-bounce diamond / ZnSe ATR accessory. Data is presented as frequency of absorption $\left(\mathrm{cm}^{-1}\right)$. All melting points were determined using a Fisher-Johns melting point apparatus. High resolution mass spectra were obtained from SI2 Micromass 70S-250 mass spectrometer (EI) or an AB SCIEX QSTAR ${ }_{\circledast}$ mass spectrometer (ESI) or a JEOL ${ }_{\otimes}$ AccuTOF medel JMS-T1000LC mass spectrometer equipped with an IONICS $\otimes_{\circledast}$ Direct Analysis in real Time (DART) ion source. 


\section{Optimization of the Reaction Conditions}

\section{Table S1. Optimization of the Reaction Conditions ${ }^{\mathrm{a}}$}

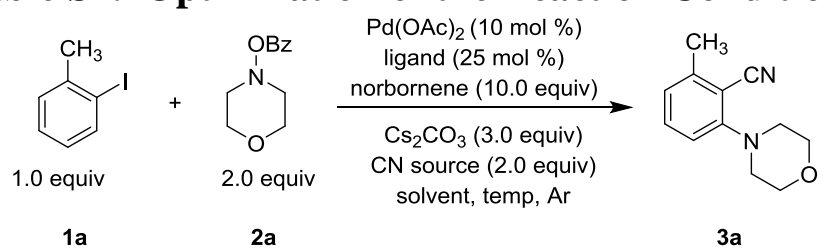

\begin{tabular}{|c|c|c|c|c|c|}
\hline entry & CN source & ligand & solvent & $\mathrm{T}\left({ }^{\circ} \mathrm{C}\right)$ & yield $(\%)^{b}$ \\
\hline 1 & $\mathrm{~K}_{4}\left[\mathrm{Fe}(\mathrm{CN})_{6}\right]$ & $\mathrm{PPh}_{3}$ & toluene & 100 & 15 \\
\hline 2 & CuCN & $\mathrm{PPh}_{3}$ & toluene & 100 & 5 \\
\hline 3 & $\mathrm{KCN}$ & $\mathrm{PPh}_{3}$ & toluene & 100 & 43 \\
\hline 4 & $\mathrm{Zn}(\mathrm{CN})_{2}$ & $\mathrm{PPh}_{3}$ & toluene & 100 & $83(82)$ \\
\hline 5 & $\mathrm{Zn}(\mathrm{CN})_{2}$ & $\mathrm{PPh}_{3}$ & $\mathrm{CH}_{3} \mathrm{CN}$ & 100 & 5 \\
\hline 6 & $\mathrm{Zn}(\mathrm{CN})_{2}$ & $\mathrm{PPh}_{3}$ & DME & 100 & 58 \\
\hline 7 & $\mathrm{Zn}(\mathrm{CN})_{2}$ & $\mathrm{PPh}_{3}$ & DCE & 100 & 68 \\
\hline 8 & $\mathrm{Zn}(\mathrm{CN})_{2}$ & $\mathrm{PPh}_{3}$ & dioxane & 100 & 80 \\
\hline 9 & $\mathrm{Zn}(\mathrm{CN})_{2}$ & $\mathrm{PPh}_{3}$ & DMF & 100 & 5 \\
\hline 10 & $\mathrm{Zn}(\mathrm{CN})_{2}$ & $\left(4-\mathrm{MeOC}_{6} \mathrm{H}_{4}\right)_{3} \mathrm{P}$ & toluene & 100 & 72 \\
\hline 11 & $\mathrm{Zn}(\mathrm{CN})_{2}$ & (4-tolyl) ${ }_{3} \mathrm{P}$ & toluene & 100 & 77 \\
\hline 12 & $\mathrm{Zn}(\mathrm{CN})_{2}$ & $(2 \text {-tolyl) })_{3} \mathrm{P}$ & toluene & 100 & 23 \\
\hline 13 & $\mathrm{Zn}(\mathrm{CN})_{2}$ & $(3 \text {-tolyl) })_{3} \mathrm{P}$ & toluene & 100 & $83(79)$ \\
\hline 14 & $\mathrm{Zn}(\mathrm{CN})_{2}$ & $(2 \text {-furyl) })_{3} \mathrm{P}$ & toluene & 100 & 72 \\
\hline $15^{c}$ & $\mathrm{Zn}(\mathrm{CN})_{2}$ & $\mathrm{PPh}_{3}$ & toluene & 100 & 60 \\
\hline $16^{a}$ & $\mathrm{Zn}(\mathrm{CN})_{2}$ & $\mathrm{PPh}_{3}$ & toluene & 100 & 76 \\
\hline 17 & $\mathrm{Zn}(\mathrm{CN})_{2}$ & $\mathrm{PPh}_{3}$ & toluene & 110 & 68 \\
\hline 18 & $\mathrm{Zn}(\mathrm{CN})_{2}$ & $\mathrm{PPh}_{3}$ & toluene & 120 & $92(89)$ \\
\hline 19 & $\mathrm{Zn}(\mathrm{CN})_{2}$ & $\mathrm{PPh}_{3}$ & toluene & 130 & $88(86)$ \\
\hline $20^{e}$ & $\mathrm{Zn}(\mathrm{CN})_{2}$ & $\mathrm{PPh}_{3}$ & toluene & 120 & 65 \\
\hline $21^{\dagger}$ & $\mathrm{Zn}(\mathrm{CN})_{2}$ & $\mathrm{PPh}_{3}$ & toluene & 120 & 80 \\
\hline
\end{tabular}

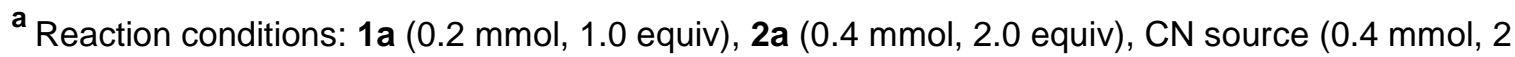
equiv), $\mathrm{Pd}\left(10 \mathrm{~mol} \%\right.$ ), ligand ( $25 \mathrm{~mol} \%$ ), norbornene (2 mmol, 10.0 equiv), $\mathrm{Cs}_{2} \mathrm{CO}_{3}$ (0.6 mmol, 3.0 equiv), solvent (3.0 mL), $24 \mathrm{~h}$, argon. ${ }^{\mathbf{b}}$ Determined by ${ }^{1} \mathrm{H}$ NMR spectroscopy using 1,3,5trimethoxybenzene as an internal standard. Isolated yield after column chromatography are given in parentheses. ${ }^{c}$ With 3.0 equiv of Norbornene. ${ }^{d}$ With 5.0 equiv of Norbornene. ${ }^{\mathbf{e}}$ With 1.0 equiv of $\mathrm{Zn}(\mathrm{CN})_{2}$ as $\mathrm{CN}$ source. ${ }^{\mathrm{f}}$ With 3.0 equiv of $\mathrm{Zn}(\mathrm{CN})_{2}$ as $\mathrm{CN}$ source. 


\section{Detailed Experimental Procedures Synthesis of O-Benzoyl Hydroxylamines}

O-benzoyl-hydroxylamines were prepared by literature procedure. ${ }^{1}$

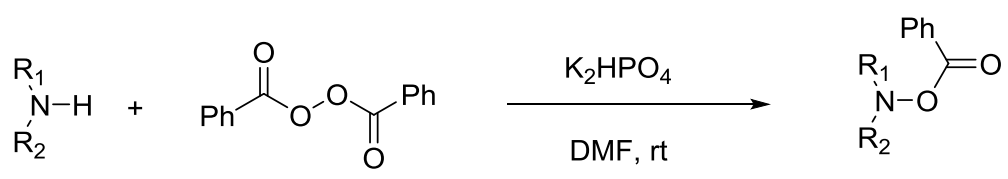

A 50-mL, one-necked, round-bottomed flask equipped with a Teflon-coated magnetic stir bar was charged with benzoyl peroxide $(2.42 \mathrm{~g}, 10 \mathrm{mmol}), \mathrm{K}_{2} \mathrm{HPO}_{4}(2.61 \mathrm{~g}, 15 \mathrm{mmol})$, and $\mathrm{N}$, N-dimethyl formamide $(25 \mathrm{~mL})$. The suspension was stirred and the amine $(12 \mathrm{mmol})$ was added via syringe in one portion. The suspension was stirred at ambient temperature for the indicated reaction time. Deionized water $(25 \mathrm{~mL})$ was added and the contents were stirred vigorously for several minutes until all solids dissolved. The reaction mixture was transferred to a $100 \mathrm{~mL}$ separatory funnel and extracted with $25 \mathrm{~mL}$ of ethyl acetate. The organic phase was collected and washed with two $25 \mathrm{~mL}$ portions of saturated aq $\mathrm{NaHCO}_{3}$ solution. All of the aqueous fractions were combined and extracted with three $25 \mathrm{~mL}$ portions of ethyl acetate. All of the organic fractions were combined and washed with three 100 $\mathrm{mL}$ portions of deionized water, $25 \mathrm{~mL}$ of brine, dried over $\mathrm{Na}_{2} \mathrm{SO}_{4}$, and concentrated by rotary evaporation. The resulting crude product mixture was purified by flash column chromatography, eluting with the indicated solvent system, to afford desired product of $\geq 95 \%$ purity as judged by ${ }^{1} \mathrm{H}$ NMR spectroscopy. The product was stored at sub-ambient temperature or room temperature (if the product is solid) under anhydrous conditions.<smiles>[R16][NH+]1CCOCC1</smiles>

Morpholino benzoate (2a): white solid, (1.41 g, 68\% yield). ${ }^{1} \mathrm{H}$ NMR (400 MHz, CDCl3) $\delta 8.02-$ $7.99(\mathrm{~m}, 2 \mathrm{H}), 7.58-7.54(\mathrm{~m}, 1 \mathrm{H}), 7.46-7.42(\mathrm{~m}, 2 \mathrm{H}), 3.99-3.95(\mathrm{~m}, 2 \mathrm{H}), 3.88-3.83(\mathrm{~m}, 2 \mathrm{H}), 3.45$ - 3.43 (m, 2H), 3.06 - 3.01 (m, 2H). ${ }^{13} \mathrm{C} \mathrm{NMR} \mathrm{(100} \mathrm{MHz,} \mathrm{CDCl3)} \mathrm{164.7,} \mathrm{133.3,} \mathrm{129.5,} \mathrm{129.3,} \mathrm{128.6,}$ 66.0, 57.1 .

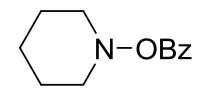

Piperidin-1-yl benzoate (2q): white solid, (1.68 g, 82\% yield). ${ }^{1} \mathrm{H}$ NMR (400 MHz, CDCl3) $\delta 8.01-$ $7.99(\mathrm{~m}, 2 \mathrm{H}), 7.56-7.52(\mathrm{~m}, 1 \mathrm{H}), 7.44-7.40(\mathrm{~m}, 2 \mathrm{H}), 3.51-3.49(\mathrm{~m}, 2 \mathrm{H}), 2.78-2.76(\mathrm{~m}, 2 \mathrm{H}), 1.86$ $-1.79(\mathrm{~m}, 4 \mathrm{H}), 1.69-1.66(\mathrm{~m}, 1 \mathrm{H}), 1.31-1.25(\mathrm{~m}, 1 \mathrm{H}) .{ }^{13} \mathrm{C}$ NMR (100 MHz, CDCl3) 164.9, 133.0, $129.8,129.5,128.5,57.6,25.1,23.5$.

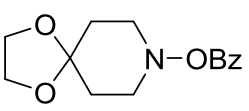


1,4-dioxa-8-azaspiro[4.5] decan-8-yl benzoate (2r): white solid, $\left(2.11 \mathrm{~g}, 80 \%\right.$ yield). ${ }^{1} \mathrm{H}$ NMR $(400 \mathrm{MHz}, \mathrm{CDCl} 3) \delta 8.02-7.99(\mathrm{~m}, 2 \mathrm{H}), 7.58-7.54(\mathrm{~m}, 1 \mathrm{H}),, 7.46-7.41(\mathrm{~m}, 2 \mathrm{H}), 3.99(\mathrm{~s}, 4 \mathrm{H})$, $3.44-3.26(\mathrm{~m}, 4 \mathrm{H}), 1.96(\mathrm{t}, J=5.9 \mathrm{~Hz}, 1 \mathrm{H}) .{ }^{13} \mathrm{C} \mathrm{NMR}(100 \mathrm{MHz}, \mathrm{CDCl} 3) 164.9,133.2,129.57$, 129.55, 128.6, 106.2, 64.6, 54.1, 32.7 .

BzO-N

Tert-butyl 4-(benzoyloxy)piperazine-1-carboxylate (2s): white solid, $\left(2.14 \mathrm{~g}, 70 \%\right.$ yield). ${ }^{1} \mathrm{H}$ NMR (400 MHz, CDCl3) $\delta 8.01-7.99(\mathrm{~m}, 2 \mathrm{H}), 7.59-7.55(\mathrm{~m}, 1 \mathrm{H}), 7.47-7.42(\mathrm{~m}, 2 \mathrm{H}), 4.03(\mathrm{~s}, 2 \mathrm{H}), 3.44$ $-3.32(\mathrm{~m}, 4 \mathrm{H}), 2.92-2.90(\mathrm{~m}, 2 \mathrm{H}), 1.48$ (s, 9H). ${ }^{13} \mathrm{C}$ NMR (100 MHz, CDCl3) 164.7, 154.6, 133.4, 129.6, 129.3, 128.6, 80.4, 56.0, 28.5.

$\mathrm{C}^{\mathrm{N}-\mathrm{OBz}}$

Pyrrolidin-1-yl benzoate (2t): white solid, (1.19 g, 62\% yield). ${ }^{1} \mathrm{H}$ NMR (400 MHz, CDCl3) $\delta 7.98-$ $7.95(\mathrm{~m}, 2 \mathrm{H}), 7.56-7.52(\mathrm{~m}, 1 \mathrm{H}), 7.44-7.40(\mathrm{~m}, 2 \mathrm{H}), 3.32-3.29(\mathrm{~m}, 4 \mathrm{H}), 1.96(\mathrm{~s}, 4 \mathrm{H}) .{ }^{13} \mathrm{C}$ NMR (100 MHz, CDCl3) 165.3, 133.0, 129.7, 129.5, 128.5, 57.9, 22.3.

$\mathrm{Me}^{-} \mathrm{N}^{-\mathrm{Bn}}$
$\mathrm{OBz}$

O-benzoyl-N-benzyl-N-methylhydroxylamine (2u): white solid, (2.05 g, 85\% yield). ${ }^{1} \mathrm{H}$ NMR (400 $\mathrm{MHz}, \mathrm{CDCl} 3) \delta 7.92-7.89(\mathrm{~m}, 2 \mathrm{H}), 7.55-7.50(\mathrm{~m}, 1 \mathrm{H}), 7.44-7.37(\mathrm{~m}, 4 \mathrm{H}), 7.33-7.24(\mathrm{~m}, 2 \mathrm{H})$, 4.17 (s, 2H), 2.93 (s, 3H). ${ }^{13} \mathrm{C}$ NMR (100 MHz, CDCl3) 165.1, 135.8, 133.1, 129.5, 129.47, 129.46, $128.5,128.46,127.9,65.2,46.2$.

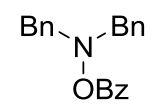

O-benzoyl-N,N-dibenzylhydroxylamine (2v): white solid, (2.22 g, 70\% yield). ${ }^{1} \mathrm{H}$ NMR (400 MHz, $\mathrm{CDCl} 3) \delta 7.85-7.82(\mathrm{~m}, 2 \mathrm{H}), 7.52-7.48(\mathrm{~m}, 1 \mathrm{H}), 7.47-7.44(\mathrm{~m}, 4 \mathrm{H}), 7.39-7.24(\mathrm{~m}, 8 \mathrm{H}), 4.21(\mathrm{~s}$, 4H). ${ }^{13} \mathrm{C}$ NMR (100 MHz, CDCl3) 165.0, 136.1, 132.9, 129.5, 129.48, 129.41, 128.5, 128.4, 127.8, 62.2 .<smiles>CCN([O])CC</smiles>

O-benzoyl-N,N-diethylhydroxylamine (2w): white solid, (1.2 g, 62\% yield). ${ }^{1} \mathrm{H}$ NMR (400 MHz, $\mathrm{CDCl} 3) \delta 8.05-8.02(\mathrm{~m}, 2 \mathrm{H}), 7.58-7.54(\mathrm{~m}, 1 \mathrm{H}), 7.46-7.42(\mathrm{~m}, 2 \mathrm{H}), 3.04(\mathrm{q}, J=7.1 \mathrm{~Hz}, 4 \mathrm{H}), 1.18$ (t, $J=7.1 \mathrm{~Hz}, 6 \mathrm{H}) .{ }^{13} \mathrm{C}$ NMR (100 MHz, CDCl3) 166.0, 133.1, 129.6, 129.4, 128.5, 53.6, 12.0 . 


\section{Synthesis of Aryl Iodides}

Aryl Iodides 1a, 1b, 1e, 1f, 1h, 1k, 1l, 1o, 1p, 4a-i, 4k, $4 \mathbf{l}$ were commercially available

from Aldrich, Combi-Blocks, Alfa Aesar. Iodides 1c, $\mathbf{1 d}, \mathbf{1 g}, \mathbf{1 i}, \mathbf{1 j}, \mathbf{1 m}, \mathbf{1 n}$ and $\mathbf{4 j}$ synthesis were previously reported by literature procedure:

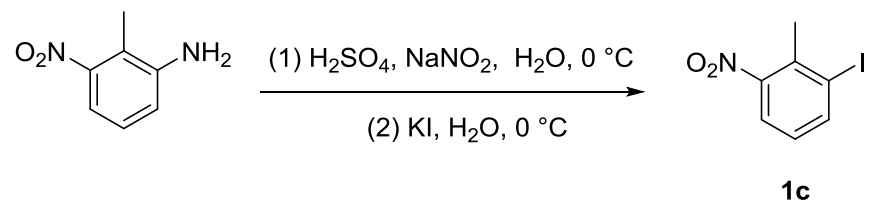

2-Methyl-3-nitro-iodobenzene (1c) ${ }^{2}$. To a solution of 2-methyl-3-nitroaniline (3.00 g, $19.7 \mathrm{mmol}, 1.0$ equiv) in water $(20 \mathrm{~mL})$ was added a solution of $\mathrm{H}_{2} \mathrm{SO}_{4}(4 \mathrm{~mL})$ in water $(20 \mathrm{~mL})$. The mixture was cooled to $0{ }^{\circ} \mathrm{C}$, then a solution of sodium nitrite $(1.43 \mathrm{~g}, 20.72 \mathrm{mmol}, 1.05 \mathrm{mmol})$ in water $(20 \mathrm{~mL})$ was added dropwise. The mixture was stirred for $1 \mathrm{~h}$, then a solution of potassium iodide (4.91 g, 29.6 mmol, 1.5 equiv ) in water $(20 \mathrm{~mL})$ was added dropwise. The reaction was stirred for $1 \mathrm{~h}$ and then extracted with $\mathrm{CH}_{2} \mathrm{Cl}_{2}(3 \times)$. The combined organic extracts were washed with saturated aqueous $\mathrm{Na}_{2} \mathrm{~S}_{2} \mathrm{O}_{3}$, dried with anhydrous $\mathrm{Na}_{2} \mathrm{SO}_{4}$, filtered and concentrated. The crude mixture was purified by flash chromatography using 5\% DCM/hexanes as eluant to yield $(4.2 \mathrm{~g}, 81 \%)$ as a yellow solid. ${ }^{1} \mathrm{H}$ NMR (400 MHz, $\left.\mathrm{CDCl}_{3}\right) \delta 8.07-8.05(\mathrm{~m}, 1 \mathrm{H}), 7.73-7.71(\mathrm{~m}, 1 \mathrm{H}), 7.06-7.01(\mathrm{~m}, 1 \mathrm{H}), 2.60(\mathrm{~s}, 3 \mathrm{H})$. ${ }^{13} \mathrm{C}$ NMR (100 MHz, $\mathrm{CDCl}_{3}$ ) 143.3, 135.2, 128.1, 124.1, 110.2, 103.7, 25.1.

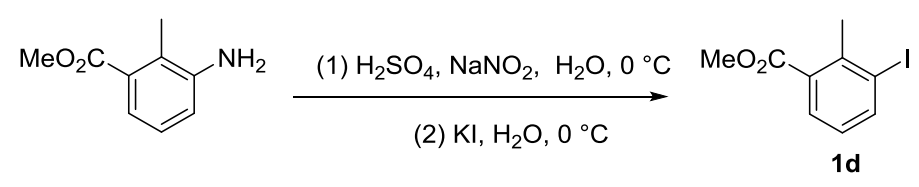

Methyl 3-iodo-2-methylbenzoate (1d $)^{2}$. To a solution of methyl-3-amino-2-methylbenzoate $(3.6 \mathrm{~mL}$, $25 \mathrm{mmol}, 1.0$ equiv) in water $(25 \mathrm{~mL})$ was added a solution of $\mathrm{H}_{2} \mathrm{SO}_{4}(5 \mathrm{~mL})$ in water $(25 \mathrm{~mL})$. The mixture was cooled to $0{ }^{\circ} \mathrm{C}$, then a solution of sodium nitrite $(1.81 \mathrm{~g}, 26.5 \mathrm{mmol}, 1.06 \mathrm{mmol})$ in water $(25 \mathrm{~mL})$ was added dropwise. The mixture was stirred for $1 \mathrm{~h}$, then a solution of potassium iodide (6.23 g, $37.5 \mathrm{mmol}, 1.5$ equiv) in water $(25 \mathrm{~mL})$ was added dropwise. The reaction was stirred for $1 \mathrm{~h}$ and then extracted with $\mathrm{CH}_{2} \mathrm{Cl}_{2}(3 \times)$. The combined organic extracts were washed with saturated aqueous $\mathrm{Na}_{2} \mathrm{~S}_{2} \mathrm{O}_{3}$, dried with anhydrous $\mathrm{Na}_{2} \mathrm{SO}_{4}$, filtered and concentrated. The crude mixture was purified by flash chromatography using 20\% DCM/hexanes as eluant to yield 5 (5.98 g, 87\%) as a colourless solid. ${ }^{1} \mathrm{H}$ NMR $\left(400 \mathrm{MHz}, \mathrm{CDCl}_{3}\right) \delta 7.99-7.96(\mathrm{~m}, 1 \mathrm{H}), 7.75-7.72(\mathrm{~m}, 1 \mathrm{H}), 6.94-6.90$ $(\mathrm{m}, 1 \mathrm{H}), 3.90(\mathrm{~s}, 3 \mathrm{H}), 2.66(\mathrm{~s}, 3 \mathrm{H}) .{ }^{13} \mathrm{C} \mathrm{NMR}\left(100 \mathrm{MHz}, \mathrm{CDCl}_{3}\right)$ 168.1 142.7, 141.6, 132.0, 130.1, 127.2, 104.2, 52.4, 26.6 .

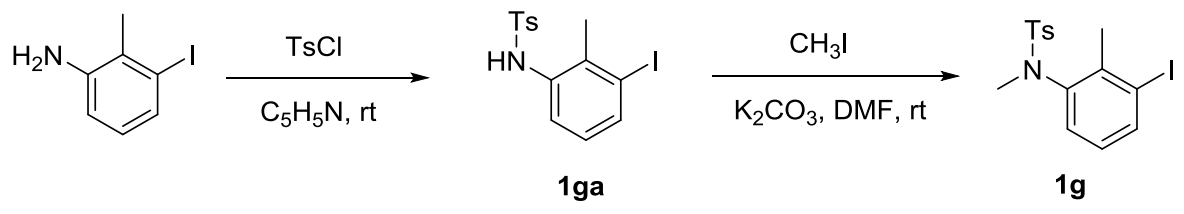


$\boldsymbol{N}$-(3-iodo-2-methylphenyl)-4-methylbenzenesulfonamide (1ga) ${ }^{3}$. 3-iodo-2-methylaniline (597 mg, $2.56 \mathrm{mmol}$ ) and $\mathrm{TsCl}(586 \mathrm{mg}, 3.07 \mathrm{mmol})$ were combined and dissolved in $20 \mathrm{~mL}$ dry pyridine. The mixture was capped and stirred for $24 \mathrm{~h}$ at $70{ }^{\circ} \mathrm{C}$. The mixture was concentrated in vacuo, poured into in $15 \mathrm{~mL} 10 \%$ hydrochloric acid in water, and extracted with 3 x $20 \mathrm{~mL}$ EtOAc. The combined organic phases were then washed with saturated $\mathrm{NaHCO}_{3}$, then dried with $\mathrm{MgSO}_{4}$, filtered and concentrated. The crude mixture was purified by flash chromatography using $20 \%$ EtOAc/hexanes as eluant to yield 1 ga (746 mg, 75\%) as a white solid. ${ }^{1} \mathrm{H} \mathrm{NMR}\left(400 \mathrm{MHz}, \mathrm{CDCl}_{3}\right) \delta 7.68-7.66(\mathrm{~m}, 1 \mathrm{H})$, $7.63-7.61(\mathrm{~m}, 2 \mathrm{H}), 7.27-7.23(\mathrm{~m}, 3 \mathrm{H}), 6.82(\mathrm{t}, J=8.0 \mathrm{~Hz}, 1 \mathrm{H}), 6.72(\mathrm{~s}, 1 \mathrm{H}), 2.42(\mathrm{~s}, 3 \mathrm{H}), 2.21(\mathrm{~s}$, $3 \mathrm{H}) .{ }^{13} \mathrm{C} \mathrm{NMR}\left(100 \mathrm{MHz}, \mathrm{CDCl}_{3}\right)$ 144.2, 137.7, 136.5, 135.9, 134.4, 129.9, 128.2, 127.3, 125.5, 102.2, 23.7, 21.7.

$\boldsymbol{N}$-(3-iodo-2-methylphenyl)-N,4-dimethylbenzenesulfonamide (1g) ${ }^{2}$. To a solution of $\mathbf{1 g a}(697 \mathrm{mg}$, $1.80 \mathrm{mmol}, 1.0$ equiv) in DMF $(10 \mathrm{~mL})$ was added $\mathrm{K}_{2} \mathrm{CO}_{3}(746 \mathrm{mg}, 5.4 \mathrm{mmol}, 3.0$ equiv) and iodomethane $(0.22 \mathrm{ml}, 3.6 \mathrm{mmol}, 2.0$ equiv). The reaction was stirred at $\mathrm{rt}$ overnight then quenched with water $(20 \mathrm{~mL})$. The aqueous layer was extracted with EtOAc $(3 \times)$ and the combined organic extracts were dried with anhydrous $\mathrm{Na}_{2} \mathrm{SO}_{4}$, filtered and concentrated. The crude mixture was purified by flash chromatography using $10 \%$ EtOAc/hexanes as eluant to yield $\mathbf{1 g}$ (704 $\mathrm{mg}, 98 \%)$ as a yellow oil. ${ }^{1} \mathrm{H}$ NMR (400 MHz, $\left.\mathrm{CDCl}_{3}\right) \delta 7.79-7.77(\mathrm{~m}, 2 \mathrm{H}), 7.59-7.57(\mathrm{~m}, 2 \mathrm{H}), 7.32-7.29(\mathrm{~m}, 2 \mathrm{H}), 6.77$ - $6.73(\mathrm{~m}, 1 \mathrm{H}), 6.61-6.59(\mathrm{~m}, 1 \mathrm{H}), 3.10(\mathrm{~s}, 3 \mathrm{H}), 2.52(\mathrm{~s}, 3 \mathrm{H}), 2.45(\mathrm{~s}, 3 \mathrm{H}) .{ }^{13} \mathrm{C} \mathrm{NMR}(100 \mathrm{MHz}$, $\left.\mathrm{CDCl}_{3}\right)$ 143.9, 142.8, 140.5, 139.4, 134.9, 129.7, 128.1, 127.7, 127.3, 102.8, 39.2, 24.7, 21.7.
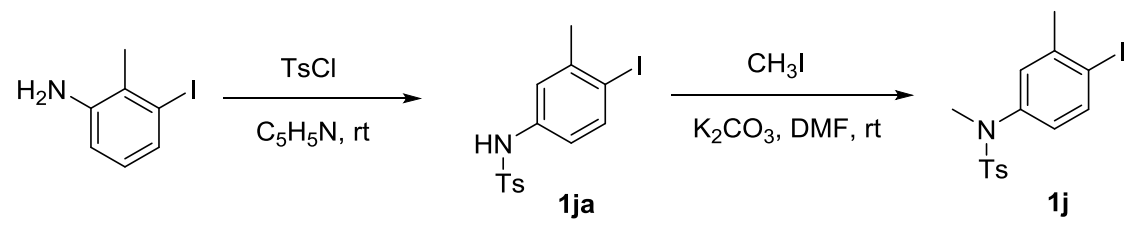

$N$-(4-iodo-3-methylphenyl)-4-methylbenzenesulfonamide (1ja) ${ }^{2}$. To a solution of 4-methyl-3iodoaniline $(1.50 \mathrm{~g}, 6.44 \mathrm{mmol}, 1$ equiv) in pyridine $(20 \mathrm{~mL})$ was added in one portion $\mathrm{TsCl}(1.29 \mathrm{~g}$, $6.76 \mathrm{mmol}, 1.05$ equiv). The reaction was stirred at $\mathrm{rt}$ overnight then The reaction mixture was poured into $10 \%$ solution of hydrochloric acid in water $(200 \mathrm{~mL})$, extracted with EtOAc $(3 \mathrm{x} 50 \mathrm{~mL})$. The combined organic fractions were washed with $10 \%$ aqueous hydrochloric acid (100 ml), water (100 $\mathrm{mL}$ ), and saturated solution of $\mathrm{NaHCO}_{3}$. The crude mixture was purified by flash chromatography using $10 \%$ EtOAc/hexanes as eluant to yield $\mathbf{1 j a}(2.25 \mathrm{~g}, 90 \%)$ as a white solid. ${ }^{1} \mathrm{H} \mathrm{NMR}(400 \mathrm{MHz}$, $\left.\mathrm{CDCl}_{3}\right) \delta 7.70-7.68(\mathrm{~m}, 1 \mathrm{H}), 7.60(\mathrm{~d}, J=8.5 \mathrm{~Hz}, 1 \mathrm{H}), 7.25-7.20(\mathrm{~m}, 3 \mathrm{H}), 6.98-6.97(\mathrm{~m}, 1 \mathrm{H}), 6.66$ - $6.63(\mathrm{~m}, 3 \mathrm{H}), 6.72(\mathrm{~s}, 1 \mathrm{H}), 2.38(\mathrm{~s}, 3 \mathrm{H}), 2.31$ (s, 3H). ${ }^{13} \mathrm{C} \mathrm{NMR}\left(100 \mathrm{MHz}, \mathrm{CDCl}_{3}\right)$ 144.3, 142.7, 139.6, 137.0, 135.9, 129.9, 127.4, 122.4, 120.1, 96.1, 28.2, 21.7.

$\mathbf{N}$-(4-iodo-3-methylphenyl)-N,4-dimethylbenzenesulfonamide $(\mathbf{1 j})^{2}$. To a solution of $\mathbf{1 j a}(1.94 \mathrm{~g}$, $5.0 \mathrm{mmol}, 1.0$ equiv) in DMF $(20 \mathrm{~mL})$ was added $\mathrm{K}_{2} \mathrm{CO}_{3}(2.07 \mathrm{~g}, 15.0 \mathrm{mmol}, 3.0$ equiv) and iodomethane $(0.62 \mathrm{ml}, 10.0 \mathrm{mmol}, 2.0$ equiv). The reaction was stirred at $\mathrm{rt}$ overnight then quenched with water $(20 \mathrm{~mL})$. The aqueous layer was extracted with EtOAc $(3 \times)$ and the combined organic extracts were dried with anhydrous $\mathrm{Na}_{2} \mathrm{SO}_{4}$, filtered and concentrated. The crude mixture was purified by flash chromatography using $10 \%$ EtOAc/hexanes as eluant to yield $\mathbf{1 j}$ (1.98 g, 99\%) as a white 
solid. ${ }^{1} \mathrm{H}$ NMR $\left(400 \mathrm{MHz}, \mathrm{CDCl}_{3}\right) \delta 7.69(\mathrm{~d}, J=8.4 \mathrm{~Hz}, 1 \mathrm{H}), 7.45-7.43(\mathrm{~m}, 2 \mathrm{H}), 7.27-7.24(\mathrm{~m}$, 2H), $7.06-7.05(\mathrm{~m}, 1 \mathrm{H}), 6.56-6.53(\mathrm{~m}, 1 \mathrm{H}), 3.11(\mathrm{~s}, 3 \mathrm{H}), 2.43(\mathrm{~s}, 3 \mathrm{H}), 2.38(\mathrm{~s}, 3 \mathrm{H}) .{ }^{13} \mathrm{C}$ NMR $(100$ $\left.\mathrm{MHz}, \mathrm{CDCl}_{3}\right)$ 143.9, 142.4, 142.0, 139.3, 133.5, 129.6, 128.4, 128.0, 125.1, 99.4, 38.1, 28.3, 21.7.

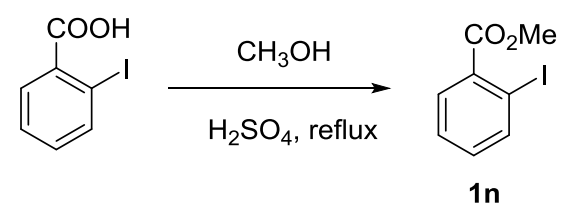

Methyl 2-iodobenzoate (1n) ${ }^{4}$. To a solution of 2-iodobenzoic acid (2.48 g, $\left.10 \mathrm{mmol}\right)$ in methanol (30 $\mathrm{mL}$ ) was added concentrated $\mathrm{H}_{2} \mathrm{SO}_{4}(3.0 \mathrm{~mL})$ cautiously and the resulting solution refluxed for $3 \mathrm{~h}$ and allowed to cool to rt. The reaction mixture was diluted with diethyl ether $(30 \mathrm{~mL})$ and then washed with $\mathrm{H}_{2} \mathrm{O}(2 \times 30 \mathrm{~mL})$, saturated $\mathrm{NaHCO}_{3}$ solution $(30 \mathrm{~mL})$, and brine $(30 \mathrm{~mL})$. The organic layer was dried over $\mathrm{Na}_{2} \mathrm{SO}_{4}$ and concentrated to get a pale yellow oil $(1.96 \mathrm{~g}, 75 \%) .{ }^{1} \mathrm{H}$ NMR $(400 \mathrm{MHz}$, $\left.\mathrm{CDCl}_{3}\right) \delta 8.00-7.98(\mathrm{~m}, 2 \mathrm{H}), 7.81-7.78(\mathrm{~m}, 2 \mathrm{H}), 7.42-7.38(\mathrm{~m}, 2 \mathrm{H}), 7.17-7.13(\mathrm{~m}, 1 \mathrm{H}), 3.93(\mathrm{~s}$, $3 \mathrm{H}) .{ }^{13} \mathrm{C} \mathrm{NMR}\left(100 \mathrm{MHz}, \mathrm{CDCl}_{3}\right)$ 167.1, 141.4, 135.3, 132.8, 131.1, 128.0, 94.2, 52.6.

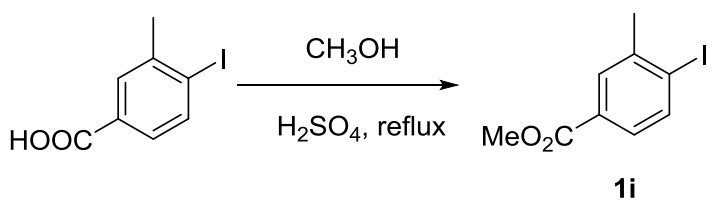

Methyl 4-iodo-3-methylbenzoate (1i) ${ }^{4}$. A solution of 3-iodo-4-methylbenzoic acid $(1.31 \mathrm{~g}, 5.00$ mmol) and $\mathrm{H}_{2} \mathrm{SO}_{4}(\sim 1.0 \mathrm{~mL})$ in $\mathrm{MeOH}(30 \mathrm{~mL})$ was refluxed overnight. The reaction mixture was cooled and the solvent was evaporated. The residue was dissolved in $\mathrm{Et}_{2} \mathrm{O}$, washed with saturated $\mathrm{NaHCO}_{3}$ (3x), $\mathrm{H}_{2} \mathrm{O}$, brine, dried over $\mathrm{Na}_{2} \mathrm{SO}_{4}$. The crude mixture was purified by flash chromatography using 5\% EtOAc/hexanes as eluant to yield $\mathbf{1 i}(1.25 \mathrm{~g}, 91 \%)$ as white solid. ${ }^{1} \mathrm{H}$ NMR $\left(400 \mathrm{MHz}, \mathrm{CDCl}_{3}\right) \delta 7.90-7.87(\mathrm{~m}, 2 \mathrm{H}), 7.51-7.48(\mathrm{~m}, 1 \mathrm{H}), 3.90(\mathrm{~s}, 3 \mathrm{H}), 2.47(\mathrm{~s}, 3 \mathrm{H}) .{ }^{13} \mathrm{C} \mathrm{NMR}$ $\left(100 \mathrm{MHz}, \mathrm{CDCl}_{3}\right)$ 166.9, 142.0, 139.3, 130.5, 130.3, 128.2, 107.6, 52.4, 28.2.

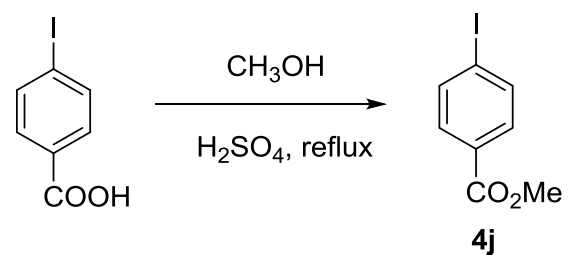

Methyl 4-iodobenzoate (4j) ${ }^{4}$. To a solution of 4-iodobenzoic acid (1.24 g, $\left.5.0 \mathrm{mmol}\right)$ in methanol (20 $\mathrm{mL}$ ) was added concentrated $\mathrm{H}_{2} \mathrm{SO}_{4}(1.5 \mathrm{~mL})$ cautiously and the resulting solution refluxed for $3 \mathrm{~h}$ and allowed to cool to rt. The reaction mixture was diluted with diethyl ether $(30 \mathrm{~mL})$ and then washed with $\mathrm{H}_{2} \mathrm{O}(2 \times 30 \mathrm{~mL})$, saturated $\mathrm{NaHCO}_{3}$ solution $(30 \mathrm{~mL})$, and brine $(30 \mathrm{~mL})$. The organic layer was dried $\left(\mathrm{Na}_{2} \mathrm{SO}_{4}\right)$ and concentrated in vacuo. The crude mixture was purified by flash chromatography using $10 \%$ EtOAc/hexanes as eluant to yield as pale yellow solid $(1.10 \mathrm{~g}, 84 \%) .{ }^{1} \mathrm{H}$ NMR (400 MHz, $\left.\mathrm{CDCl}_{3}\right) \delta 7.81-7.79(\mathrm{~m}, 2 \mathrm{H}), 7.76-7.73(\mathrm{~m}, 2 \mathrm{H}), 3.91(\mathrm{~s}, 3 \mathrm{H}) .{ }^{13} \mathrm{C} \mathrm{NMR}\left(100 \mathrm{MHz}, \mathrm{CDCl}_{3}\right) 166.7$, 137.9, 131.2, 129.8, 100.8, 52.5. 


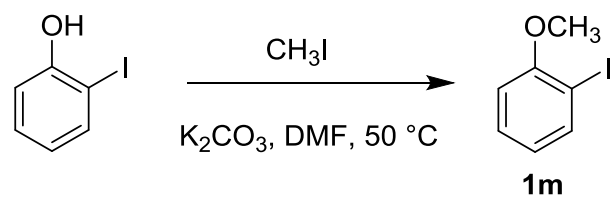

1-iodo-2-methoxybenzene (1m) ${ }^{5}$. To a solution of 2-iodophenol (5.0 mmol, 1.0 equiv) in dry DMF $(20 \mathrm{~mL})$, potassium carbonate $(25.0 \mathrm{mmol}, 5.0$ equiv) was added under stirring. After $10 \mathrm{~min}$ the iodomethane ( $7.5 \mathrm{mmol}, 1.5$ equiv) was added to the reaction mixture. The reaction was stirred at 50 ${ }^{\circ} \mathrm{C}$ for $3 \mathrm{~h}$. The reaction was quenched with water $(20 \mathrm{~mL})$. The aqueous layer was extracted with EtOAc $(3 \times)$ and the combined organic extracts were dried with anhydrous $\mathrm{Na}_{2} \mathrm{SO}_{4}$, filtered and concentrated. The crude mixture was purified by flash chromatography using $10 \%$ EtOAc/hexanes as eluant to yield $1 \mathrm{~m}(1.1 \mathrm{~g}, 95 \%)$ as a yellow oil. ${ }^{1} \mathrm{H}$ NMR $\left(400 \mathrm{MHz}, \mathrm{CDCl}_{3}\right) \delta 7.79-7.76(\mathrm{~m}, 1 \mathrm{H})$, $7.33-7.29(\mathrm{~m}, 1 \mathrm{H}), 6.85-6.82(\mathrm{~m}, 1 \mathrm{H}), 6.73-6.69(\mathrm{~m}, 1 \mathrm{H}), 3.88(\mathrm{~s}, 3 \mathrm{H}) .{ }^{13} \mathrm{C} \mathrm{NMR}(100 \mathrm{MHz}$, $\left.\mathrm{CDCl}_{3}\right)$ 158.2, 139.6, 129.7, 122.6, 111.1, 86.1, 56.4.

General Procedure for the Sequential Amination/Cyanation Reaction between Aryl Iodides 1, $N$ benzoyloxyamine 2 , and $\mathrm{Zn}(\mathrm{CN})_{2}$.

A $25 \mathrm{~mL}$ of Schlenk tube equipped with a Teflon-coated magnetic stir bar was charged with aryl iodide ( $0.2 \mathrm{mmol}, 1.0$ equiv), $N$-benzoyloxyamine ( $0.4 \mathrm{mmol}, 2.0$ equiv), $\mathrm{Zn}(\mathrm{CN})_{2}(0.4 \mathrm{mmol}, 2.0$ equiv), $\mathrm{Pd}(\mathrm{OAc})_{2}$ (4.5 mg, $\left.0.02 \mathrm{mmol}, 10 \mathrm{~mol} \%\right), \mathrm{PPh}_{3}(13.1 \mathrm{mg}, 0.05 \mathrm{mmol}, 25 \mathrm{~mol} \%$ ), norbornene (188.3 mg, $2.0 \mathrm{mmol}, 10.0$ equiv), $\mathrm{Cs}_{2} \mathrm{CO}_{3}$ (195.5 mg, $0.6 \mathrm{mmol}, 3.0$ equiv), and toluene (3.0 $\mathrm{mL}$ ). The resulting light-yellow suspension was stirred at room temperature for 10 min under argon and then heated to $120^{\circ} \mathrm{C}$ for $24 \mathrm{~h}$. The resulting mixture was cooled to room temperature and filtered through Celite with EtOAc as the eluent. The solvents were evaporated under reduced pressure, and the residue was purified by flash chromatography on silica gel using Hexanes/ethyl acetate as eluent to give the corresponding products 3a-w.

General Procedure for the Sequential Amination/Cyanation Reaction between Aryl Iodides 4, $N$ benzoyloxyamine $2 \mathrm{a}$, and $\mathrm{Zn}(\mathrm{CN})_{2}$.

A $25 \mathrm{~mL}$ of Schlenk tube equipped with a Teflon-coated magnetic stir bar was charged with aryl iodide ( $0.2 \mathrm{mmol}, 1.0$ equiv), $N$-benzoyloxyamine ( $0.5 \mathrm{mmol}, 2.5$ equiv), $\mathrm{Zn}(\mathrm{CN})_{2}(0.4 \mathrm{mmol}, 2.0$ equiv), $\mathrm{Pd}(\mathrm{OAc})_{2}$ (4.5 mg, $\left.0.02 \mathrm{mmol}, 10 \mathrm{~mol} \%\right), \mathrm{PPh}_{3}(13.1 \mathrm{mg}, 0.05 \mathrm{mmol}, 25 \mathrm{~mol} \%$ ), norbornene (188.3 mg, $2.0 \mathrm{mmol}, 10.0$ equiv), $\mathrm{Cs}_{2} \mathrm{CO}_{3}(325.8 \mathrm{mg}, 1.0 \mathrm{mmol}, 5.0$ equiv), and toluene (3.0 mL). The resulting light-yellow suspension was stirred at room temperature for 10 min under argon and then heated to $120{ }^{\circ} \mathrm{C}$ for $24 \mathrm{~h}$. The resulting mixture was cooled to room temperature and filtered through Celite with EtOAc as the eluent. The solvents were evaporated under reduced pressure, and the residue was purified by flash chromatography on silica gel using Hexanes/ethyl acetate as eluent to give the corresponding products 5a-l. 


\section{Characterization Data}<smiles>Cc1cccc(N2CCOCC2)c1C#N</smiles>

\section{2-Methyl-6-morpholinobenzonitrile (3a):}

White solid (36 mg, 89\% yield); melting point: $62-64{ }^{\circ} \mathrm{C} .{ }^{1} \mathbf{H} \mathbf{N M R}\left(400 \mathrm{MHz}, \mathrm{CDCl}_{3}\right) \delta 7.39-7.35$ $(\mathrm{m}, 1 \mathrm{H}), 6.93-6.91(\mathrm{~m}, 1 \mathrm{H}), 6.85-6.83(\mathrm{~m}, 1 \mathrm{H}), 3.90-3.88(\mathrm{~m}, 4 \mathrm{H}), 3.17-3.15(\mathrm{~m}, 4 \mathrm{H}), 2.51(\mathrm{~s}$, 3H). ${ }^{13} \mathrm{C}$ NMR $\left(100 \mathrm{MHz}, \mathrm{CDCl}_{3}\right) \delta 156.3,144.1,133.2,123.8,117.4,115.9,107.3,67.1,52.2,21.0$. IR (ATR): 2983, 2900, 2853, 2825, 2209, 1575, 1473, 1448, 1377, 1240, 1111, 868, 787. HRMS (DART): $m / z$ calculated for $\mathrm{C}_{12} \mathrm{H}_{15} \mathrm{~N}_{2} \mathrm{O}[\mathrm{M}+\mathrm{H}]: 203.11844$, found 203.11855.<smiles>Cc1ccc(N2CCOCC2)c(C#N)c1C</smiles>

\section{2,3-Dimethyl-6-morpholinobenzonitrile (3b):}

White solid (38 mg, 88\% yield); melting point: $60-62{ }^{\circ} \mathrm{C} .{ }^{1} \mathbf{H} \mathbf{N M R}\left(400 \mathrm{MHz}, \mathrm{CDCl}_{3}\right) \delta 7.25(\mathrm{~d}, J=$ $8.1 \mathrm{~Hz}, 1 \mathrm{H}), 6.78(\mathrm{~d}, J=8.3 \mathrm{~Hz}, 1 \mathrm{H}), 3.90-3.87(\mathrm{~m}, 4 \mathrm{H}), 3.12-3.09$ (m, 4H), 2.44 (s, 3H), 2.24 (s, $3 \mathrm{H}) .{ }^{13} \mathrm{C}$ NMR $\left(100 \mathrm{MHz}, \mathrm{CDCl}_{3}\right) \delta 154.5,142.1,134.6,131.4,117.8,116.1,108.2,67.2,52.5,19.5$, 18.4. IR (ATR): 3010, 2957, 2932. 2830, 2218, 1593, 1482, 1447, 1374, 1239, 1209, 1113, 1010, 889, 826. HRMS (DART): $m / z$ calculated for $\mathrm{C}_{13} \mathrm{H}_{17} \mathrm{~N}_{2} \mathrm{O}[\mathrm{M}+\mathrm{H}]: 217.13409$, found 217.13349.<smiles>Cc1c(N2CCOCC2)ccc([N+](=O)[O-])c1C#N</smiles>

2-Methyl-6-morpholino-3-nitrobenzonitrile (3c):

Yellow solid (45 mg, 91\% yield); melting point: $88-90{ }^{\circ} \mathrm{C}^{\mathbf{1}} \mathbf{H} \mathbf{~ N M R}\left(400 \mathrm{MHz}, \mathrm{CDCl}_{3}\right) \delta 8.12(\mathrm{~d}, J$ $=9.2 \mathrm{~Hz}, 1 \mathrm{H}), 6.89(\mathrm{~d}, J=9.2 \mathrm{~Hz}, 1 \mathrm{H}), 3.98-3.85(\mathrm{~m}, 4 \mathrm{H}), 3.45-3.26(\mathrm{~m}, 4 \mathrm{H}), 2.80(\mathrm{~s}, 3 \mathrm{H}) .{ }^{13} \mathbf{C}$ NMR $\left(100 \mathrm{MHz}, \mathrm{CDCl}_{3}\right) \delta 159.2,142.8,140.9,130.3,116.3,115.9,107.3,66.7,51.5,19.3$. IR (ATR): 3020, 2973, 2925, 2853, 2221, 1577, 1504, 1446, 1377, 1330, 1241, 1111, 998, 840. HRMS (DART): $m / z$ calculated for $\mathrm{C}_{12} \mathrm{H}_{14} \mathrm{~N}_{3} \mathrm{O}_{3}[\mathrm{M}+\mathrm{H}]: 248.10352$, found 248.10333. 


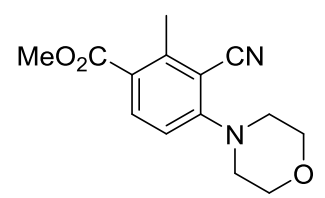

Methyl 3-cyano-2-methyl-4-morpholinobenzoate (3d):

White solid (46 mg, 88\% yield); melting point: $114-116{ }^{\circ} \mathrm{C} .{ }^{1} \mathbf{H}$ NMR $\left(400 \mathrm{MHz}, \mathrm{CDCl}_{3}\right) \delta 8.03(\mathrm{~d}$, $J=8.8 \mathrm{~Hz}, 1 \mathrm{H}), 6.85-6.83(\mathrm{~m}, 1 \mathrm{H}), 3.91-3.89(\mathrm{~m}, 4 \mathrm{H}), 3.88(\mathrm{~s}, 3 \mathrm{H}), 3.29-3.27(\mathrm{~m}, 4 \mathrm{H}), 2.80(\mathrm{~s}$, $3 \mathrm{H}) .{ }^{13} \mathrm{C}$ NMR $\left(100 \mathrm{MHz}, \mathrm{CDCl}_{3}\right) \delta 166.6,158.7,146.9,136.0,123.7,117.2,115.4,108.0,66.9,52.2$, 51.7, 20.0. IR (ATR): 2997, 2954, 2868, 2847, 2217, 1716, 1586, 1438, 1266, 1234, 1146, 1113, 1002, 834, 782. HRMS (DART): $\mathrm{m} / z$. calculated for $\mathrm{C}_{14} \mathrm{H}_{17} \mathrm{~N}_{2} \mathrm{O}_{3}[\mathrm{M}+\mathrm{H}]: 261.12392$, found 261.12384.<smiles>Cc1c(F)ccc(N2CCOCC2)c1C#N</smiles>

\section{3-Fluoro-2-methyl-6-morpholinobenzonitrile (3e):}

White solid (39 mg, 89\% yield); melting point: $78-80{ }^{\circ} \mathrm{C} .{ }^{1} \mathbf{H}$ NMR $\left(400 \mathrm{MHz}, \mathrm{CDCl}_{3}\right) \delta 7.16$ (t, $J=$ $8.8 \mathrm{~Hz}, 1 \mathrm{H}), 6.85-6.82(\mathrm{~m}, 1 \mathrm{H}), 3.90-3.87(\mathrm{~m}, 4 \mathrm{H}), 3.10-3.08(\mathrm{~m}, 4 \mathrm{H}), 2.43(\mathrm{~s}, 3 \mathrm{H}) .{ }^{13} \mathbf{C}$ NMR $\left(100 \mathrm{MHz}, \mathrm{CDCl}_{3}\right) \delta 156.40(\mathrm{~d}, J=242.0 \mathrm{~Hz}), 152.64(\mathrm{~d}, J=2.8 \mathrm{~Hz}), 130.55(\mathrm{~d}, J=20.4 \mathrm{~Hz}), 120.18$ $(\mathrm{d}, J=23.5 \mathrm{~Hz}), 117.49$ (d, $J=8.2 \mathrm{~Hz}), 116.21(\mathrm{~d}, J=3.7 \mathrm{~Hz}), 109.58(\mathrm{~d}, J=5.9 \mathrm{~Hz}), 67.1,52.6,13.6$. IR (ATR): 3020, 2964, 2867, 2845, 2222, 1479, 1444, 1263, 1239, 1112, 1000, 898, 826. HRMS (DART): $\mathrm{m} / z$ calculated for $\mathrm{C}_{12} \mathrm{H}_{14} \mathrm{FN}_{2} \mathrm{O}[\mathrm{M}+\mathrm{H}]: 221.10902$, found 221.10850 .<smiles>Cc1c(Cl)ccc(N2CCOCC2)c1C#N</smiles>

\section{3-Chloro-2-methyl-6-morpholinobenzonitrile (3f):}

White solid (44 mg, 93\% yield); melting point: $90-92{ }^{\circ} \mathrm{C} .{ }^{\mathbf{1}} \mathbf{H} \mathbf{N M R}\left(400 \mathrm{MHz}, \mathrm{CDCl}_{3}\right) \delta 7.44$ (d, $J=$ $8.8 \mathrm{~Hz}, 1 \mathrm{H}), 6.81(\mathrm{dd}, J=8.8,0.6 \mathrm{~Hz}, 1 \mathrm{H}), 3.90-3.87(\mathrm{~m}, 4 \mathrm{H}), 3.15-3.13(\mathrm{~m}, 4 \mathrm{H}), 2.56(\mathrm{~s}, 3 \mathrm{H}) .{ }^{13} \mathbf{C}$ NMR $\left(100 \mathrm{MHz}, \mathrm{CDCl}_{3}\right) \delta 155.1,141.4,133.9,128.2,117.4,116.7,109.1,67.0,52.2,19.2$. IR (ATR): 2969, 2893, 2860, 2829, 2222, 1576, 1459, 1444, 1239, 1114, 990, 870, 824, 689. HRMS (DART): $\mathrm{m} / z$ calculated for $\mathrm{C}_{12} \mathrm{H}_{14} \mathrm{ClN}_{2} \mathrm{O}[\mathrm{M}+\mathrm{H}]: 237.07947$, found 237.07905 . 


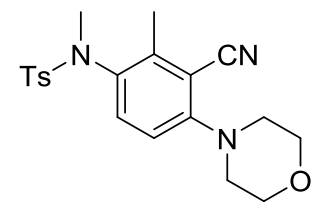

N-(3-cyano-2-methyl-4-morpholinophenyl)-N,4-dimethylbenzenesulfonamide (3g):

White solid (68 mg, 88\% yield); melting point: $166-168{ }^{\circ} \mathrm{C} .{ }^{1} \mathbf{H} \mathbf{N M R}\left(400 \mathrm{MHz}, \mathrm{CDCl}_{3}\right) \delta 7.57(\mathrm{~d}$, $J=8.3 \mathrm{~Hz}, 2 \mathrm{H}), 7.33-7.30(\mathrm{~m}, 2 \mathrm{H}), 6.77(\mathrm{~d}, J=8.7 \mathrm{~Hz}, 1 \mathrm{H}), 6.69(\mathrm{dd}, J=8.8,0.6 \mathrm{~Hz}, 1 \mathrm{H}), 3.87(\mathrm{t}, J$ $=4.6 \mathrm{~Hz}, 3 \mathrm{H}), 3.19-3.14(\mathrm{~m}, 2 \mathrm{H}), 3.08(\mathrm{~s}, 3 \mathrm{H}), 2.51(\mathrm{~s}, 3 \mathrm{H}), 2.45(\mathrm{~s}, 3 \mathrm{H}) .{ }^{13} \mathrm{C}$ NMR $(100 \mathrm{MHz}$, $\left.\mathrm{CDCl}_{3}\right) \delta 155.8,145.2,144.0,134.9,134.8,132.4,129.8,128.0,116.9,116.4,108.6,67.0,52.0,39.0$, 21.7, 17.5. IR (ATR): 3000, 2956, 2924, 2852, 2216, 1590, 1485, 1448, 1339, 1243, 1161, 1119, 999, 818, 669. HRMS (ESI): $m / z$ calculated for $\mathrm{C}_{20} \mathrm{H}_{24} \mathrm{~N}_{3} \mathrm{O}_{3} \mathrm{~S}[\mathrm{M}+\mathrm{H}]: 386.1533$, found 386.1528 .<smiles>Cc1cc([N+](=O)[O-])cc(N2CCOCC2)c1C#N</smiles>

\section{2-Methyl-6-morpholino-4-nitrobenzonitrile (3h):}

Yellow solid (40 mg, 81\% yield); melting point: $167-169{ }^{\circ} \mathrm{C} .{ }^{1} \mathbf{H}$ NMR $\left(400 \mathrm{MHz}, \mathrm{CDCl}_{3}\right) \delta 7.73$ $(\mathrm{dd}, J=2.0,0.8 \mathrm{~Hz}, 1 \mathrm{H}), 7.67-7.63(\mathrm{~m}, 1 \mathrm{H}), 3.93-3.90(\mathrm{~m}, 4 \mathrm{H}), 3.29-3.27(\mathrm{~m}, 4 \mathrm{H}), 2.44(\mathrm{~s}, 3 \mathrm{H})$, 2.62 (s, 3H). ${ }^{13} \mathrm{C}$ NMR (100 MHz, $\left.\mathrm{CDCl}_{3}\right) \delta 157.0,150.6,146.1,117.6,115.9,112.0,110.8,66.8$, 51.9, 21.4. IR (ATR): 3072, 2969, 2957, 2870, 2215, 1584, 1525, 1434, 1347, 1257, 1116, 990, 866. HRMS (DART): $m / z$ calculated for $\mathrm{C}_{12} \mathrm{H}_{14} \mathrm{~N}_{3} \mathrm{O}_{3}[\mathrm{M}+\mathrm{H}]: 248.10352$, found 248.10359.

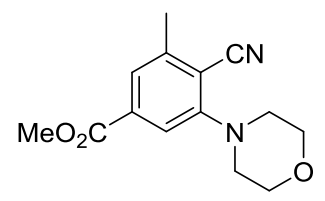

\section{Methyl 4-cyano-3-methyl-5-morpholinobenzoate (3i):}

White solid (38 mg, 73\% yield); melting point: $94-96{ }^{\circ} \mathrm{C} .{ }^{1} \mathbf{H} \mathbf{N M R}\left(400 \mathrm{MHz}, \mathrm{CDCl}_{3}\right) \delta 7.57(\mathrm{dd}, J$ $=1.4,0.8 \mathrm{~Hz}, 1 \mathrm{H}), 7.48-7.47(\mathrm{~m}, 2 \mathrm{H}), 3.92(\mathrm{~s}, 3 \mathrm{H}), 3.91-3.89(\mathrm{~m}, 4 \mathrm{H}), 3.22-3.20(\mathrm{~m}, 4 \mathrm{H}), 2.55$ (s, 3H). ${ }^{13} \mathrm{C}$ NMR $\left(100 \mathrm{MHz}, \mathrm{CDCl}_{3}\right) \delta$ 166.1, 156.2, 144.4, 134.2, 124.4, 116.8, 116.7, 110.0, 67.0, 52.7, 52.1, 21.1. IR (ATR): 2984, 2957, 2913, 2859, 2835, 2221, 1724, 1569, 1426, 1377, 1278, 1267 , 1230, 1113, 983, 866, 765. HRMS (DART): $\mathrm{m} / \mathrm{z}$. calculated for $\mathrm{C}_{14} \mathrm{H}_{17} \mathrm{~N}_{2} \mathrm{O}_{3}[\mathrm{M}+\mathrm{H}]: 261.12392$, found 261.12412. 


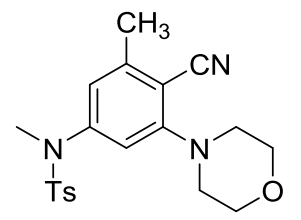

$N$-(4-cyano-3-methyl-5-morpholinophenyl)-N,4-dimethylbenzenesulfonamide (3j):

White solid (70 mg, 91\% yield); melting point: $126-128{ }^{\circ} \mathrm{C} .{ }^{1} \mathbf{H} \mathbf{~ N M R}\left(400 \mathrm{MHz}, \mathrm{CDCl}_{3}\right) \delta 7.44(\mathrm{~d}$, $J=8.3 \mathrm{~Hz}, 1 \mathrm{H}), 7.27-7.24(\mathrm{~m}, 2 \mathrm{H}), 6.68(\mathrm{~d}, J=2.0 \mathrm{~Hz}, 1 \mathrm{H}), 6.57-6.56(\mathrm{~m}, 1 \mathrm{H}), 3.87-3.84(\mathrm{~m}$, $4 \mathrm{H}), 3.13(\mathrm{~s}, 3 \mathrm{H}), 3.12-3.10(\mathrm{~m}, 4 \mathrm{H}) 3.22-3.20(\mathrm{~m}, 4 \mathrm{H}), 2.43(\mathrm{~s}, 3 \mathrm{H}), 2.42(\mathrm{~s}, 3 \mathrm{H}) .{ }^{\mathbf{1 3}} \mathbf{C}$ NMR $(100$ $\left.\mathrm{MHz}, \mathrm{CDCl}_{3}\right) \delta 156.5,146.1,144.7,144.3,133.5,129.6,127.8,119.9,117.0,114.1,105.0,67.0,52.0$, 37.6, 21.7, 21.1. IR (ATR): 2964, 2957, 2926, 2850, 2217, 1591, 1571, 1440, 1347, 1170, 1122, 956, 863, 659. HRMS (DART): $m / z$ calculated for $\mathrm{C}_{20} \mathrm{H}_{24} \mathrm{~N}_{3} \mathrm{O}_{3} \mathrm{~S}[\mathrm{M}+\mathrm{H}]: 386.15384$, found 386.15440.

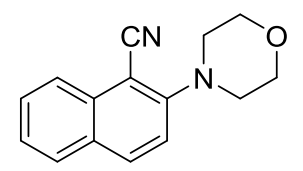

\section{2-Morpholino-1-naphthonitrile (3k):}

White solid (36 mg, 76\% yield); melting point: $131-133{ }^{\circ} \mathrm{C} .{ }^{1} \mathbf{H}$ NMR (400 MHz, $\left.\mathrm{CDCl}_{3}\right) \delta 8.14-$ $8.11(\mathrm{~m}, 1 \mathrm{H}), 7.96-7.94(\mathrm{~m}, 1 \mathrm{H}), 7.81-7.78(\mathrm{~m}, 1 \mathrm{H}), 7.64-7.59(\mathrm{~m}, 1 \mathrm{H}), 7.46-7.42(\mathrm{~m}, 1 \mathrm{H}), 7.24$ - $7.22(\mathrm{~m}, 1 \mathrm{H}), 3.96-3.94(\mathrm{~m}, 4 \mathrm{H}), 3.43-3.41(\mathrm{~m}, 4 \mathrm{H}) .{ }^{13} \mathbf{C} \mathbf{N M R}\left(100 \mathrm{MHz}, \mathrm{CDCl}_{3}\right) \delta 155.8$, 134.28, 134.27, 129.1, 128.7, 128.4, 125.4, 124.3, 118.2, 117.7, 99.1, 67.2, 52.0. IR (ATR): 2968, 2897, 2848, 2207, 1617, 1590, 1505, 1453, 1369, 1209, 1113, 978, 871, 815, 760. HRMS (DART): $m / z$ calculated for $\mathrm{C}_{15} \mathrm{H}_{15} \mathrm{~N}_{2} \mathrm{O}[\mathrm{M}+\mathrm{H}]: 239.11844$, found 239.1178 .

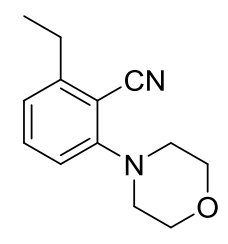

\section{2-Ethyl-6-morpholinobenzonitrile (31):}

White solid (31 mg, 72\% yield); melting point: $40-42{ }^{\circ} \mathrm{C} .{ }^{\mathbf{1}} \mathbf{H} \mathbf{N M R}\left(400 \mathrm{MHz}, \mathrm{CDCl}_{3}\right) \delta 7.41(\mathrm{t}, J=$ $7.9 \mathrm{~Hz}, 1 \mathrm{H}), 6.95(\mathrm{dd}, J=7.7,0.8 \mathrm{~Hz}, 1 \mathrm{H}), 6.86(\mathrm{dd}, J=8.3,0.9 \mathrm{~Hz}, 1 \mathrm{H}), 3.91-3.89$ (m, 4H), $3.18-$ $3.16(\mathrm{~m}, 4 \mathrm{H}), 2.84(\mathrm{q}, J=7.6 \mathrm{~Hz}, 2 \mathrm{H}), 1.28(\mathrm{t}, J=7.6 \mathrm{~Hz}, 3 \mathrm{H}) .{ }^{13} \mathbf{C} \mathbf{N M R}\left(100 \mathrm{MHz}, \mathrm{CDCl}_{3}\right) \delta 156.4$, 150.3, 133.5, 122.5, 117.2, 116.1, 106.7, 67.2, 52.4, 28.2, 15.1. IR (ATR): 2971, 2935, 2854, 2837 , 2213, 1727, 1577, 1468, 1447, 1370, 1267, 1240, 1115, 949, 808, 752. HRMS (DART): $\mathrm{m} / z$ calculated for $\mathrm{C}_{13} \mathrm{H}_{17} \mathrm{~N}_{2} \mathrm{O}[\mathrm{M}+\mathrm{H}]: 217.13409$, found 217.13361 . 


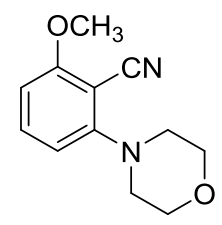

\section{Methyl 2-cyano-3-morpholinobenzoate (3m) :}

White solid (33 mg, 76\% yield); melting point: $113-115{ }^{\circ} \mathrm{C} .{ }^{1} \mathbf{H} \mathbf{~ N M R}\left(400 \mathrm{MHz}, \mathrm{CDCl}_{3}\right) \delta 7.40(\mathrm{t}, J$ $=8.4 \mathrm{~Hz}, 1 \mathrm{H}), 6.57(\mathrm{~d}, J=8.4 \mathrm{~Hz}, 2 \mathrm{H}), 3.90(\mathrm{~s}, 3 \mathrm{H}), 3.89-3.86(\mathrm{~m}, 4 \mathrm{H}), 3.21-3.19(\mathrm{~m}, 4 \mathrm{H}) .{ }^{13} \mathrm{C}$ NMR $\left(100 \mathrm{MHz}, \mathrm{CDCl}_{3}\right) \delta 163.2,157.3,134.5,116.0,110.6,104.6,95.5,67.1,56.3,51.9$. IR (ATR): 2969, 2897, 2860, 2853, 2217, 1582, 1475, 1377, 1267, 1231, 1102, 1039, 787. HRMS (DART): $\mathrm{m} / \mathrm{z}$ calculated for $\mathrm{C}_{12} \mathrm{H}_{15} \mathrm{~N}_{2} \mathrm{O}_{2}[\mathrm{M}+\mathrm{H}]$ : 219.11335, found 219.11297.<smiles>CC(=O)c1cccc(N2CCOCC2)c1N</smiles>

\section{Methyl 2-cyano-3-morpholinobenzoate (3n):}

White solid (33 mg, 67\% yield); melting point: $120-122{ }^{\circ} \mathrm{C} .{ }^{1} \mathbf{H} \mathbf{N M R}\left(400 \mathrm{MHz}, \mathrm{CDCl}_{3}\right) \delta 7.68(\mathrm{dd}$, $J=7.7,1.1 \mathrm{~Hz}, 1 \mathrm{H}), 7.58-7.54(\mathrm{~m}, 1 \mathrm{H}), 7.23(\mathrm{dd}, J=8.3,1.1 \mathrm{~Hz}, 1 \mathrm{H}), 3.97(\mathrm{~s}, 3 \mathrm{H}), 3.92-3.90(\mathrm{~m}$, 4H), $3.22-3.20(\mathrm{~m}, 4 \mathrm{H}) .{ }^{13} \mathbf{C}$ NMR $\left(100 \mathrm{MHz}, \mathrm{CDCl}_{3}\right) \delta$ 165.1, 157.6, 134.8, 133.1, 124.5, 123.0, 116.3, 106.7, 67.0, 52.9, 52.3. IR (ATR): 3010, 2964, 2853, 2850, 2218, 1720, 1587, 1452, 1432, 1270, 1214, 1112, 1003, 755. HRMS (DART): $m / z$ calculated for $\mathrm{C}_{13} \mathrm{H}_{15} \mathrm{~N}_{2} \mathrm{O}_{3}[\mathrm{M}+\mathrm{H}]: 247.10827$, found 247.10829.<smiles>N#Cc1c(F)cccc1N1CCOCC1</smiles>

\section{2-Fluoro-6-morpholinobenzonitrile (3o):}

Yellow oil (37 mg, 90\% yield). ${ }^{1} \mathbf{H}$ NMR $\left(400 \mathrm{MHz}, \mathrm{CDCl}_{3}\right) \delta 7.48-7.42(\mathrm{~m}, 1 \mathrm{H}), 6.79-6.74(\mathrm{~m}$, 2H), $3.89-3.87$ (m, 4H), $3.25-3.23(\mathrm{~m}, 4 \mathrm{H}) .{ }^{13} \mathbf{C}$ NMR (100 MHz, $\left.\mathrm{CDCl}_{3}\right) \delta 164.87(\mathrm{~d}, J=257.8$ $\mathrm{Hz}), 156.91(\mathrm{~d}, J=3.0 \mathrm{~Hz}), 134.99(\mathrm{~d}, J=10.5 \mathrm{~Hz}), 113.85(\mathrm{~d}, J=3.1 \mathrm{~Hz}), 113.53(\mathrm{~d}, J=1.8 \mathrm{~Hz})$, $108.84(\mathrm{~d}, J=20.0 \mathrm{~Hz}), 95.07$ (d, $J=16.5 \mathrm{~Hz}), 66.9,51.7$. IR (ATR): 3025, 2962, 2856, 2853, 2224, $1608,1565,1477,1448,1376,1259,1236,1115,999,789$. 


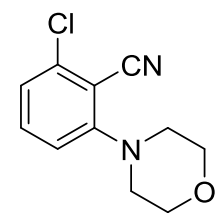

\section{2-Chloro-6-morpholinobenzonitrile (3p):}

White solid (28 mg, 63\% yield); melting point: $88-90{ }^{\circ} \mathrm{C} .{ }^{\mathbf{1}} \mathbf{H} \mathbf{N M R}\left(400 \mathrm{MHz}, \mathrm{CDCl}_{3}\right) \delta 7.41(\mathrm{t}, J=$ $8.2 \mathrm{~Hz}, 1 \mathrm{H}), 7.10(\mathrm{dd}, J=8.0,0.9 \mathrm{~Hz}, 1 \mathrm{H}), 6.90(\mathrm{dd}, J=8.4,0.9 \mathrm{~Hz}, 1 \mathrm{H}), 3.91-3.88$ (m, 4H), $3.23-$ $3.21(\mathrm{~m}, 4 \mathrm{H}) .{ }^{13} \mathbf{C}$ NMR $\left(100 \mathrm{MHz}, \mathrm{CDCl}_{3}\right) \delta 157.5,138.3,134.1,123.0,116.8,115.5,107.3,67.0$, 52.0. IR (ATR): 3092, 2975, 2920, 2866, 2841, 2229, 1587, 1460, 1438, 1376, 1243, 1111, 952, 783, 712. HRMS (DART): $m / z$. calculated for $\mathrm{C}_{11} \mathrm{H}_{12} \mathrm{ClN}_{2} \mathrm{O}[\mathrm{M}+\mathrm{H}]: 223.06382$, found 223.06382 .<smiles>Cc1cccc(N2CCCCC2)c1C#N</smiles>

\section{2-Methyl-6-(piperidin-1-yl)benzonitrile (3q):}

White solid (36 mg, 90\% yield); melting point: $46-48{ }^{\circ} \mathrm{C} .{ }^{\mathbf{1}} \mathbf{H} \mathbf{N M R}\left(400 \mathrm{MHz}, \mathrm{CDCl}_{3}\right) \delta 7.32(\mathrm{t}, J=$ $7.9 \mathrm{~Hz}, 1 \mathrm{H}), 6.83(\mathrm{dd}, J=8.1,7.1 \mathrm{~Hz}, 2 \mathrm{H}), 3.12-3.10(\mathrm{~m}, 4 \mathrm{H}), 2.49(\mathrm{~s}, 3 \mathrm{H}), 1.80-1.75(\mathrm{~m}, 4 \mathrm{H})$, $1.63-1.57(\mathrm{~m}, 2 \mathrm{H}) .{ }^{13} \mathrm{C}$ NMR $\left(100 \mathrm{MHz}, \mathrm{CDCl}_{3}\right) \delta 157.8,143.7,132.9,122.8,117.7,116.1,107.2$, 53.6, 26.3, 24.2, 21.1. IR (ATR): 3002, 2931, 2854, 2834, 2218, 1588, 1473, 1449, 1383, 1243, 963, 782, 739. HRMS (DART): $m / z$ calculated for $\mathrm{C}_{13} \mathrm{H}_{17} \mathrm{~N}_{2}$ [M+H]: 201.13917, found 201.13936.

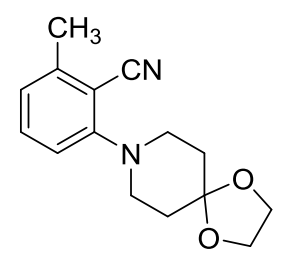

\section{2-Methyl-6-(1,4-dioxa-8-azaspiro[4.5]decan-8-yl)benzonitrile (3r):}

White solid (41 mg, 79\% yield); melting point: $46-48{ }^{\circ} \mathrm{C} .{ }^{\mathbf{1}} \mathbf{H} \mathbf{N M R}\left(400 \mathrm{MHz}, \mathrm{CDCl}_{3}\right) \delta 7.32(\mathrm{t}, J=$ $7.9 \mathrm{~Hz}, 1 \mathrm{H}), 6.88-6.84(\mathrm{~m}, 2 \mathrm{H}), 3.99(\mathrm{~s}, 4 \mathrm{H}), 3.27-3.24(\mathrm{~m}, 4 \mathrm{H}), 2.49(\mathrm{~s}, 3 \mathrm{H}), 1.94-1.92(\mathrm{~m}, 4 \mathrm{H})$. ${ }^{13}$ C NMR $\left(100 \mathrm{MHz} \mathrm{CDCl}_{3}\right) \delta$ 156.8, 143.8, 133.0, 123.3, 117.5, 116.4, 107.4, 107.0, 64.5, 50.5, 35.4, 21.0. IR (ATR): 2998, 2968, 2907, 2846, 2217, 1585, 1476, 1462, 1364, 1237, 1140, 1103, 1084, 1058, 907, 780. HRMS (DART): $m / z$ calculated for $\mathrm{C}_{15} \mathrm{H}_{19} \mathrm{~N}_{2} \mathrm{O}_{2}$ [M+H]: 259.14465, found 259.14468. 


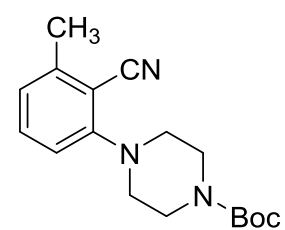

Tert-butyl 4-(2-cyano-3-methylphenyl)piperazine-1-carboxylate (3s):

Yellow oil (49 mg, 81\% yield). ${ }^{1} \mathbf{H}$ NMR $\left(400 \mathrm{MHz}, \mathrm{CDCl}_{3}\right) \delta 7.36(\mathrm{t}, J=7.9 \mathrm{~Hz}, 1 \mathrm{H}), 6.92(\mathrm{dd}, J=$ 7.6, $0.8 \mathrm{~Hz}, 1 \mathrm{H}), 6.82(\mathrm{~d}, J=8.2 \mathrm{~Hz}, 1 \mathrm{H}), 3.65-3.62(\mathrm{~m}, 4 \mathrm{H}), 3.12-3.09(\mathrm{~m}, 4 \mathrm{H}), 2.51(\mathrm{~s}, 3 \mathrm{H}), 1.48$ (s, 9H). ${ }^{13} \mathbf{C}$ NMR $\left(100 \mathrm{MHz}, \mathrm{CDCl}_{3}\right) \delta 156.3,154.9,144.0,133.2,123.9,117.3,116.3,107.7,80.0$, 52.0, 43.9, 28.6, 21.0. IR (ATR): 3020, 2976, 2929, 2862, 2216, 1690, 1589, 1474, 1417, 1365, 1237, 1163, 1121, 1001, 788. HRMS (DART): $m / z$ calculated for $\mathrm{C}_{17} \mathrm{H}_{24} \mathrm{~N}_{3} \mathrm{O}_{2}[\mathrm{M}+\mathrm{H}]: 302.18685$, found 302.18768 .<smiles>Cc1cccc(N2CCCC2)c1C#N</smiles>

\section{2-Methyl-6-(pyrrolidin-1-yl)benzonitrile (3t):}

Yellow oil (14 mg, 38\% yield). ${ }^{1} \mathbf{H}$ NMR $\left(400 \mathrm{MHz}, \mathrm{CDCl}_{3}\right) \delta 7.19(\mathrm{dd}, J=8.6,7.3 \mathrm{~Hz}, 1 \mathrm{H}), 6.55$ (dt, $J=7.3,0.9 \mathrm{~Hz}, 1 \mathrm{H}), 6.49(\mathrm{~d}, J=8.7 \mathrm{~Hz}, 1 \mathrm{H}), 3.61-3.58(\mathrm{~m}, 4 \mathrm{H}), 2.46(\mathrm{~s}, 3 \mathrm{H}), 2.00-1.97(\mathrm{~m}, 4 \mathrm{H})$. ${ }^{13}$ C NMR $\left(100 \mathrm{MHz}, \mathrm{CDCl}_{3}\right) \delta 151.5,144.3,132.9,120.1,117.7,111.7,96.1,50.3,26.0,21.7$. IR (ATR): 2971, 2957, 2874, 2835, 2203, 1590, 1478, 1461, 1376, 1355, 770. HRMS (DART): $\mathrm{m} / \mathrm{z}$ calculated for $\mathrm{C}_{12} \mathrm{H}_{15} \mathrm{~N}_{2}[\mathrm{M}+\mathrm{H}]$ : 187.12352 , found 187.12332 .<smiles>Cc1cccc(N(C)Cc2ccccc2)c1C#N</smiles>

\section{2-(Benzyl(methyl)amino)-6-methylbenzonitrile (3u):}

Yellow oil (31 mg, 66\% yield). ${ }^{1} \mathbf{H}$ NMR $\left(400 \mathrm{MHz}, \mathrm{CDCl}_{3}\right) \delta 7.34-7.26(\mathrm{~m}, 6 \mathrm{H}), 6.85-6.81(\mathrm{~m}$, 2H), 4.45 (s, 2H), 2.86 (s, 3H), 2.53 (s, 3H). ${ }^{13} \mathbf{C}$ NMR (100 MHz, $\left.\mathrm{CDCl}_{3}\right) \delta$ 156.3, 144.2, 137.7, 132.9, 128.6, 128.2, 127.5, 122.2, 118.1, 116.3, 105.1, 60.2, 40.6, 21.3. IR (ATR): 3064, 3029, 2954, 2855, 2804, 2213, 1587, 1578, 1475, 1452, 1320, 1027, 780, 708, 680. HRMS (DART): $\mathrm{m} / \mathrm{z}$ calculated for $\mathrm{C}_{16} \mathrm{H}_{17} \mathrm{~N}_{2}[\mathrm{M}+\mathrm{H}]$ : 237.13917, found 237.13895.

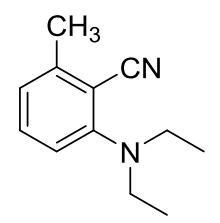




\section{2-(Diethylamino)-6-methylbenzonitrile (3w):}

Yellow oil (11 mg, 29\% yield). ${ }^{1} \mathbf{H}$ NMR $\left(400 \mathrm{MHz}, \mathrm{CDCl}_{3}\right) \delta 7.30-7.26(\mathrm{~m}, 1 \mathrm{H}), 6.83-6.78(\mathrm{~m}$, 2H), 3.32 (q, $J=7.1 \mathrm{~Hz}, 4 \mathrm{H}), 2.49$ (s, 3H), 1.13 (t, $J=7.0 \mathrm{~Hz}, 6 \mathrm{H}) .{ }^{13} \mathbf{C}$ NMR $\left(100 \mathrm{MHz}, \mathrm{CDCl}_{3}\right) \delta$ 154.8, 144.1, 132.5, 121.9, 118.3, 117.2, 106.2, 46.9, 21.3, 12.8. IR (film, NaCl): 2972, 2931, 2901, 2838, 2214, 1588, 1467, 1380, 1263, 1084, 789. HRMS (DART): $\mathrm{m} / z$ calculated for $\mathrm{C}_{12} \mathrm{H}_{17} \mathrm{~N}_{2}[\mathrm{M}+\mathrm{H}]$ : 189.13917 , found 189.13918 .<smiles>N#Cc1c(N2CCOCC2)cccc1N1CCOCC1</smiles>

\section{2,6-Dimorpholinobenzonitrile (5a):}

White solid (42 mg, 77\% yield); melting point: $118-120{ }^{\circ} \mathrm{C} .{ }^{1} \mathbf{H}$ NMR $\left(400 \mathrm{MHz}, \mathrm{CDCl}_{3}\right) \delta 7.37$ (t, $J$ $=8.2 \mathrm{~Hz}, 1 \mathrm{H}), 6.61(\mathrm{~d}, J=8.2 \mathrm{~Hz}, 2 \mathrm{H}), 3.89-3.87(\mathrm{~m}, 8 \mathrm{H}), 3.19-3.16(\mathrm{~m}, 8 \mathrm{H}) .{ }^{13} \mathbf{C}$ NMR $(100$ $\left.\mathrm{MHz}, \mathrm{CDCl}_{3}\right) \delta 157.8,134.3,117.7,111.9,100.3,67.1,52.2$. IR (ATR): 3078, 2970, 2888, 2846, 2212, 1570, 1470, 1448, 1373, 1263, 1229, 1113, 997, 805. HRMS (DART): $\mathrm{m} / \mathrm{z}$ calculated for $\mathrm{C}_{15} \mathrm{H}_{20} \mathrm{~N}_{3} \mathrm{O}_{2}[\mathrm{M}+\mathrm{H}]: 274.15555$, found 274.15527.

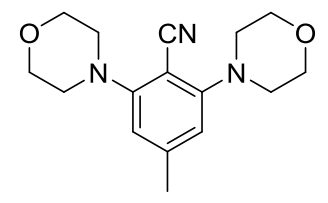

\section{4-Methyl-2,6-dimorpholinobenzonitrile (5b):}

White solid (44 mg, 77\% yield); melting point: $121-123{ }^{\circ} \mathrm{C} .{ }^{1} \mathbf{H}$ NMR (400 MHz, $\left.\mathrm{CDCl}_{3}\right) \delta 6.42$ (s, 2H), $3.89-3.86(\mathrm{~m}, 8 \mathrm{H}), 3.17-3.15(\mathrm{~m}, 8 \mathrm{H}), 2.33(\mathrm{~s}, 3 \mathrm{H}) .{ }^{13} \mathbf{C} \mathbf{N M R}\left(100 \mathrm{MHz}, \mathrm{CDCl}_{3}\right) \delta 157.7$, 145.5, 118.0, 112.8, 97.4, 67.1, 52.2, 22.6. IR (ATR): 3003, 2953, 2924, 2835, 2208, 1592, 1560, 1440, 1371, 1259, 1230, 1110, 992, 860, 839. HRMS (DART): $m / z$ calculated for $\mathrm{C}_{16} \mathrm{H}_{22} \mathrm{~N}_{3} \mathrm{O}_{2}[\mathrm{M}+\mathrm{H}]$ : 288.17120, found 288.17120 .

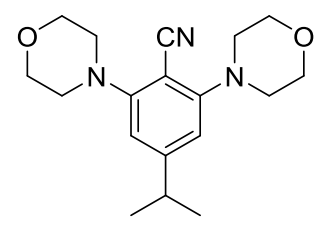

\section{4-Isopropyl-2,6-dimorpholinobenzonitrile (5c):}

White solid (43 mg, 68\% yield); melting point: $109-111{ }^{\circ} \mathrm{C} .{ }^{1} \mathbf{H}$ NMR $\left(400 \mathrm{MHz}, \mathrm{CDCl}_{3}\right) \delta 6.46$ (s, 2H), $3.89-3.87(\mathrm{~m}, 8 \mathrm{H}), 3.19-3.18(\mathrm{~m}, 8 \mathrm{H}), 2.85(\mathrm{~m}, 1 \mathrm{H}), 1.23(\mathrm{~d}, J=6.9 \mathrm{~Hz}, 6 \mathrm{H}) .{ }^{13} \mathbf{C}$ NMR $(100$ $\left.\mathrm{MHz}, \mathrm{CDCl}_{3}\right) \delta 157.9,156.4,118.0,110.2,97.8,67.2,52.3,35.3,23.7$. IR (ATR): 2997, 2953, 2890, 
2836, 2207, 1593, 1560, 1435, 1230, 1116, 1002, 861. HRMS (DART): $\mathrm{m} / \mathrm{z}$ calculated for $\mathrm{C}_{18} \mathrm{H}_{26} \mathrm{~N}_{3} \mathrm{O}_{2}[\mathrm{M}+\mathrm{H}]: 316.20250$, found 316.20246.

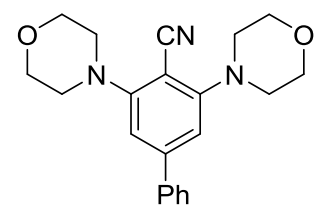

\section{3,5-Dimorpholino-[1,1'-biphenyl]-4-carbonitrile (5d):}

White solid (47 mg, 67\% yield); melting point: $168-170{ }^{\circ} \mathrm{C} .{ }^{1} \mathbf{H}$ NMR $\left(400 \mathrm{MHz}, \mathrm{CDCl}_{3}\right) \delta 7.56-$ $7.53(\mathrm{~m}, 2 \mathrm{H}), 7.49-7.41(\mathrm{~m}, 3 \mathrm{H}), 6.78(\mathrm{~s}, 2 \mathrm{H}), 3.92-3.90(\mathrm{~m}, 8 \mathrm{H}), 3.26-3.24(\mathrm{~m}, 8 \mathrm{H}) .{ }^{13} \mathbf{C} \mathbf{~ N M R}$ $\left(100 \mathrm{MHz}, \mathrm{CDCl}_{3}\right) \delta 158.0,147.7,140.4,129.1,128.7,127.4,117.8,111.0,98.8,67.1,52.3$. IR (ATR): 2966, 2937, 2887, 2849, 2209, 1592, 1552, 1420, 1234, 1117, 1006, 770, 698. HRMS (DART): $\mathrm{m} / z$ calculated for $\mathrm{C}_{21} \mathrm{H}_{24} \mathrm{~N}_{3} \mathrm{O}_{2}[\mathrm{M}+\mathrm{H}]: 350.18685$, found 350.18652 .

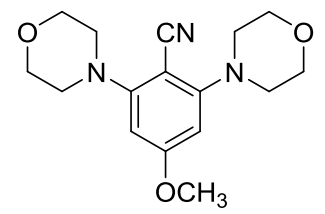

\section{4-Methoxy-2,6-dimorpholinobenzonitrile (5e):}

White solid (33 mg, 54\% yield); melting point: $138-140{ }^{\circ} \mathrm{C} .{ }^{1} \mathbf{H}$ NMR $\left(400 \mathrm{MHz}, \mathrm{CDCl}_{3}\right) \delta 6.10$ (s, 2H), $3.88-3.86(\mathrm{~m}, 8 \mathrm{H}), 3.82(\mathrm{~s}, 3 \mathrm{H}), 3.18-3.16(\mathrm{~m}, 8 \mathrm{H}) .{ }^{13} \mathbf{C} \mathbf{N M R}\left(100 \mathrm{MHz}, \mathrm{CDCl}_{3}\right) \delta$ 164.7, 159.4, 118.3, 98.0, 92.1, 67.0, 55.6, 52.1. IR (ATR): 3082, 2989, 2959, 2827, 2197, 1587, 1566, 1436, 1374, 1229, 1159, 1109, 1006, 852, 813. HRMS (DART): $\mathrm{m} / z$ calculated for $\mathrm{C}_{16} \mathrm{H}_{22} \mathrm{~N}_{3} \mathrm{O}_{3}[\mathrm{M}+\mathrm{H}]$ : 304.16612, found 304.16594.

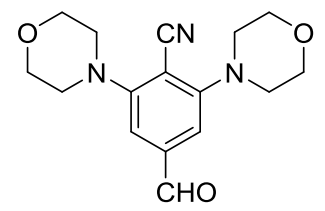

\section{4-Formyl-2,6-dimorpholinobenzonitrile (5f)}

White solid (33 mg, 54\% yield); melting point: $\left.138-140{ }^{\circ} \mathrm{C} .{ }^{1} \mathbf{H} \mathbf{~ N M R ~ ( 4 0 0 ~ M H z}, \mathrm{CDCl}_{3}\right) \delta 9.95$ (s, 1H), 7.06 (s, 2H), $3.92-3.89$ (m, 8H), $3.27-3.25(\mathrm{~m}, 8 \mathrm{H}) .{ }^{13} \mathbf{C}$ NMR $\left(100 \mathrm{MHz}, \mathrm{CDCl}_{3}\right) \delta 191.5$, 158.4, 140.3, 117.0, 112.0, 104.3, 66.9, 52.0. IR (ATR): 3082, 2961, 2854, 2837, 2213, 1698, 1565, 1436, 1374, 1257, 1223, 1112, 1006, 890, 758. HRMS (DART): $\mathrm{m} / \mathrm{z}$ calculated for $\mathrm{C}_{16} \mathrm{H}_{20} \mathrm{~N}_{3} \mathrm{O}_{3}$ $[\mathrm{M}+\mathrm{H}]: 302.15047$, found 302.15043 . 


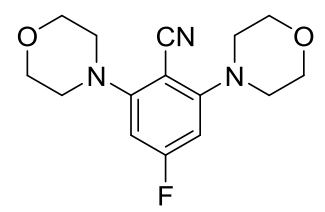

\section{4-Fluoro-2,6-dimorpholinobenzonitrile (5g):}

White solid (49 mg, 84\% yield); melting point: $103-105{ }^{\circ} \mathrm{C} .{ }^{1} \mathbf{H}$ NMR (400 MHz, $\left.\mathrm{CDCl}_{3}\right) \delta 6.27$ (d, $J=10.5 \mathrm{~Hz}, 1 \mathrm{H}), 3.88-3.86(\mathrm{~m}, 8 \mathrm{H}), 3.20-3.18(\mathrm{~m}, 8 \mathrm{H}) .{ }^{13} \mathrm{C} \mathrm{NMR}\left(100 \mathrm{MHz}, \mathrm{CDCl}_{3}\right) \delta 166.8(\mathrm{~d}, J$ $=254.0 \mathrm{~Hz}), 159.8(\mathrm{~d}, J=12.3 \mathrm{~Hz}), 117.5(\mathrm{~d}, J=1.1 \mathrm{~Hz}), 99.3(\mathrm{~d}, J=25.0 \mathrm{~Hz}), 94.9(\mathrm{~d}, J=2.8 \mathrm{~Hz})$, 66.9, 51.9. IR (ATR): 3093, 2957, 2893, 2855, 2206, 1584, 1436, 1376, 1264, 1153, 1111, 1006, 888, 800. HRMS (DART): $\mathrm{m} / z$ calculated for $\mathrm{C}_{16} \mathrm{H}_{22} \mathrm{~N}_{3} \mathrm{O}_{3}$ [M+H]: 304.16612, found 304.16594.<smiles>N#Cc1c(N2CCOCC2)cc(Cl)cc1N1CCOCC1</smiles>

\section{4-Chloro-2,6-dimorpholinobenzonitrile (5h):}

Yellow oil (54 mg, 88\% yield). ${ }^{1}$ H NMR (400 MHz, $\left.\mathrm{CDCl}_{3}\right) \delta 6.55$ (s, 2H), $3.88-3.85$ (m, 8H), 3.19 - 3.17 (m, 8H). $\left.{ }^{13} \mathbf{C ~ N M R ~ ( 1 0 0 ~ M H z , ~} \mathrm{CDCl}_{3}\right) \delta 158.4,140.8,117.3,112.2,97.7,66.9,52.0$. IR (ATR): 3080, 2961, 2893, 2857, 2209, 1562, 1428, 1226, 1112, 1004, 943, 862, 716. HRMS (DART): $\mathrm{m} / \mathrm{z}$ calculated for $\mathrm{C}_{15} \mathrm{H}_{18} \mathrm{ClN}_{3} \mathrm{O}_{2}[\mathrm{M}+\mathrm{H}]:$ 308.11660, found 308.11692.

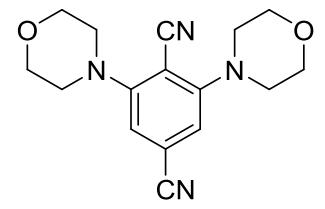

\section{2,6-Dimorpholinoterephthalonitrile (5i):}

Yellow solid (52 mg, 87\% yield); melting point: $115-117{ }^{\circ} \mathrm{C} .{ }^{1} \mathbf{H}$ NMR $\left(400 \mathrm{MHz}, \mathrm{CDCl}_{3}\right) \delta 6.78(\mathrm{~s}$, 2H), 3.88 - $3.86(\mathrm{~m}, 8 \mathrm{H}), 3.23$ - $3.20(\mathrm{~m}, 8 \mathrm{H}) .{ }^{13} \mathbf{C}$ NMR (100 MHz, $\left.\mathrm{CDCl}_{3}\right) \delta 157.9,117.9,117.6$, 116.5, 114.4, 102.8, 66.7, 52.8. IR (ATR): 3091, 2961, 2848, 2835, 2215, 2212, 1557, 1431, 1262 , 1231, 1113, 1004, 861. HRMS (DART): $m / z$ calculated for $\mathrm{C}_{16} \mathrm{H}_{19} \mathrm{~N}_{4} \mathrm{O}_{2}[\mathrm{M}+\mathrm{H}]$ : 299.15080, found 299.15090 .<smiles>CC(=O)c1cc(N2CCOCC2)c(C#N)c(N2CCOCC2)c1</smiles>

Methyl 4-cyano-3,5-dimorpholinobenzoate (5j): 
Yellow solid (50 mg, 75\% yield); melting point: $153-155{ }^{\circ} \mathrm{C} .{ }^{1} \mathbf{H}$ NMR (400 MHz, $\left.\mathrm{CDCl}_{3}\right) \delta 7.24$ (s, 2H), 3.93 (s, 3H), $3.90-3.88$ (m, 8H), $3.24-3.21$ (m, 8H). ${ }^{13} \mathbf{C}$ NMR (100 MHz, $\left.\mathrm{CDCl}_{3}\right) \delta 166.1$, 157.8, 135.3, 117.1, 112.6, 103.6, 66.7, 52.8, 52.1. IR (ATR): 2997, 2947, 2926, 2843, 2215, 1717, $1568,1428,1368,1273,1263,1112,997,860,769$. HRMS (DART): $\mathrm{m} / \mathrm{z}$ calculated for $\mathrm{C}_{17} \mathrm{H}_{22} \mathrm{~N}_{3} \mathrm{O}_{4}$ $[\mathrm{M}+\mathrm{H}]: 332.16103$, found 332.16131 .

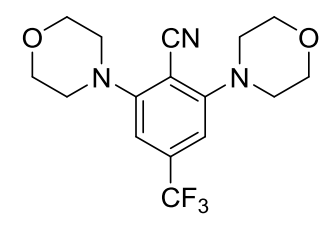

\section{2,6-Dimorpholino-4-(trifluoromethyl)benzonitrile (5k):}

White solid (57 mg, 83\% yield); melting point: $83-85{ }^{\circ} \mathrm{C} .{ }^{1} \mathbf{H}$ NMR $\left(400 \mathrm{MHz}, \mathrm{CDCl}_{3}\right) \delta 6.77$ (s, $2 \mathrm{H}), 3.90-3.88(\mathrm{~m}, 8 \mathrm{H}), 3.25-3.23(\mathrm{~m}, 8 \mathrm{H}) .{ }^{13} \mathbf{C ~ N M R}\left(100 \mathrm{MHz}, \mathrm{CDCl}_{3}\right) \delta 158.2,136.0$ (q, $J=$ $32.5 \mathrm{~Hz}), 123.4(\mathrm{q}, J=273.5 \mathrm{~Hz}), 116.9,108.1,102.08$ (d, $J=1.3 \mathrm{~Hz}), 66.9,52.0$. IR (ATR): 2969, 2957, 2860, 2833, 2217, 1578, 1435, 1375, 1293, 1226, 1164, 1107, 1001, 853, 707. HRMS (DART): $\mathrm{m} / \mathrm{z}$ calculated for $\mathrm{C}_{16} \mathrm{H}_{19} \mathrm{~F}_{3} \mathrm{~N}_{3} \mathrm{O}_{2}[\mathrm{M}+\mathrm{H}]: 342.14294$, found 342.14293 .

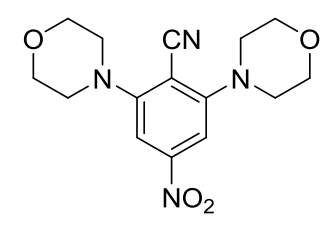

\section{2,6-Dimorpholino-4-nitrobenzonitrile (51):}

Yellow solid (40 mg, 63\% yield); melting point: $113-115{ }^{\circ} \mathrm{C} .{ }^{1} \mathbf{H}$ NMR (400 MHz, $\left.\mathrm{CDCl}_{3}\right) \delta 7.35$ (s, 2H), $3.91-3.89(\mathrm{~m}, 8 \mathrm{H}), 3.30-3.27(\mathrm{~m}, 8 \mathrm{H}) .{ }^{13} \mathbf{C} \mathbf{~ N M R}\left(100 \mathrm{MHz}, \mathrm{CDCl}_{3}\right) \delta 158.4,151.8,116.5$, 105.7, 103.5, 66.7, 51.9. IR (ATR): 3102, 2963, 2862, 2859, 2217, 1601, 1577, 1520, 1435, 1342, 1263, 1210, 1111, 1003, 864, 736. HRMS (DART): $\mathrm{m} / z$ calculated for $\mathrm{C}_{15} \mathrm{H}_{19} \mathrm{~N}_{4} \mathrm{O}_{4}[\mathrm{M}+\mathrm{H}]: 319.14063$, found 319.14112 .

\section{Reference}

(1) Berman, A.; Johnson, J. J. Org. Chem. 2006, 71, 219.

(2) Bressy, C.; Alberico, D.; Lautens, M. J. Am. Chem. Soc. 2005, 127, 13148.

(3) Martins, A.; Alberico, D.; Lautens, M. Org. Lett. 2006, 8, 4827.

(4) Kiruthika, E.; Nandakumar, A.; Perumal, P. Org. Lett. 2014, 16, 4424.

(5) Zhu, C.; Yoshimura, A.; Ji, L.; Wei, Y.; Nemykin, V.; Zhdankin, V. Org. Lett. 2012, 14, 3170. 


\section{Copies of ${ }^{1} \mathrm{H}$ and ${ }^{13} \mathrm{C}$ NMR spectra}

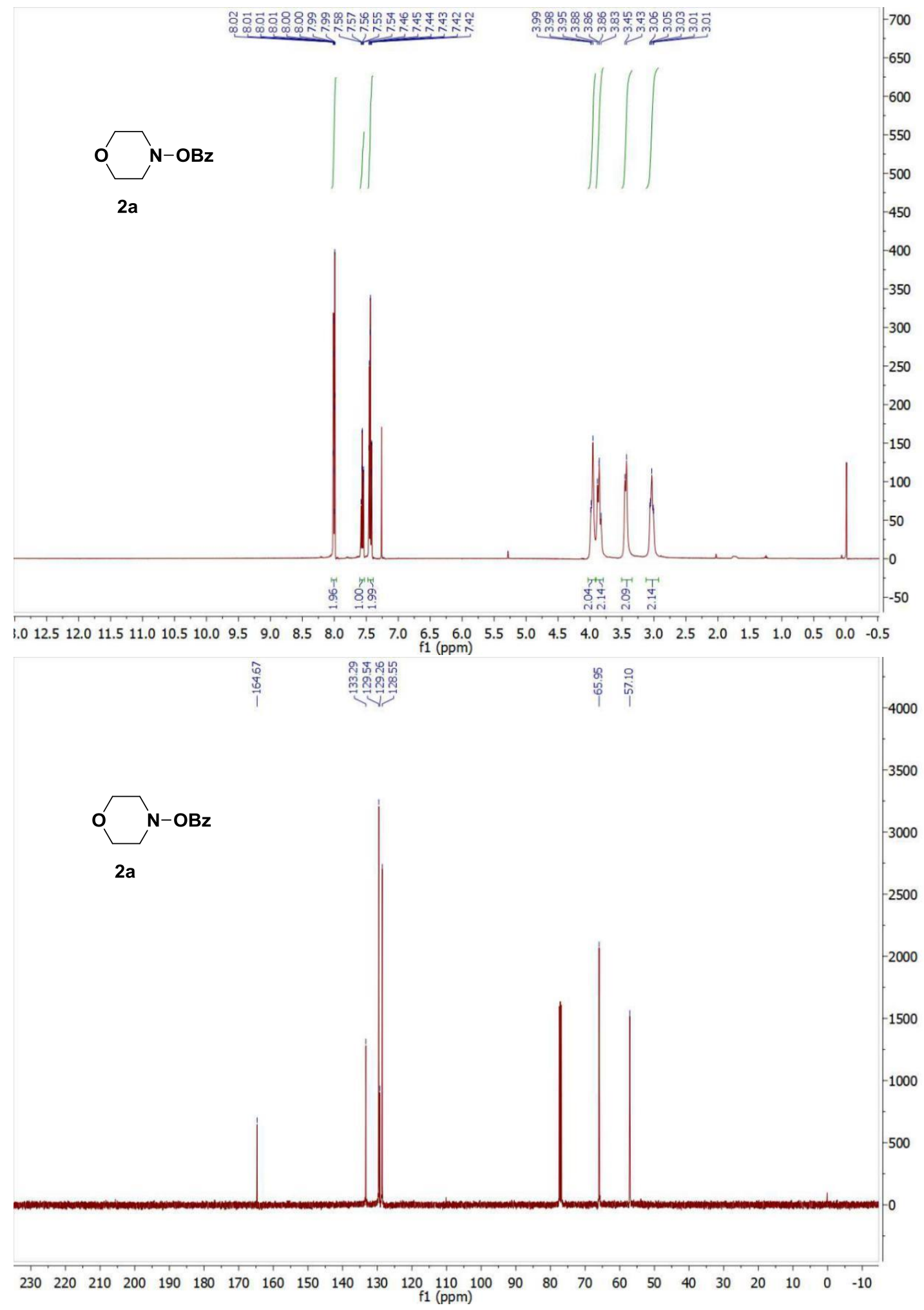




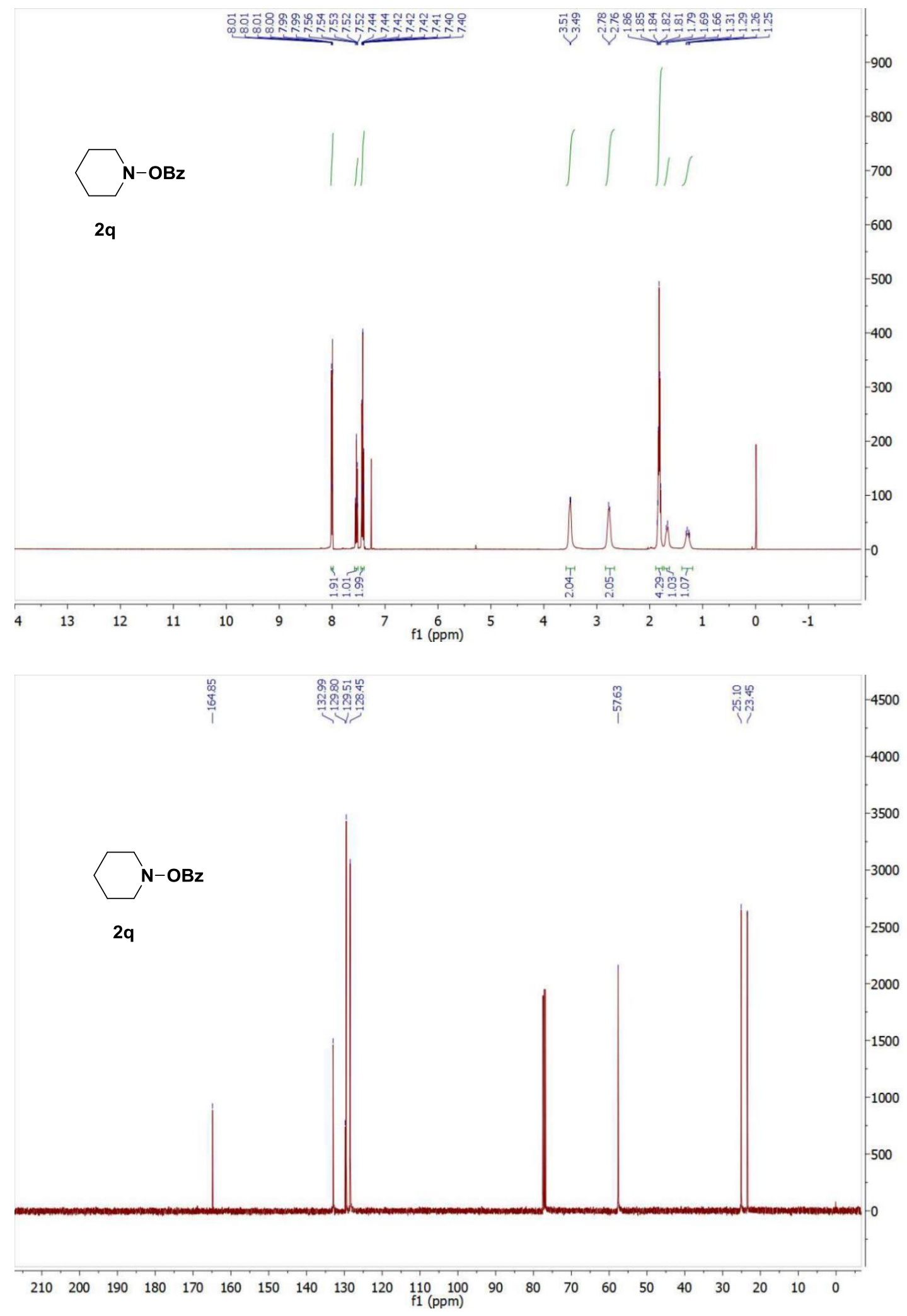




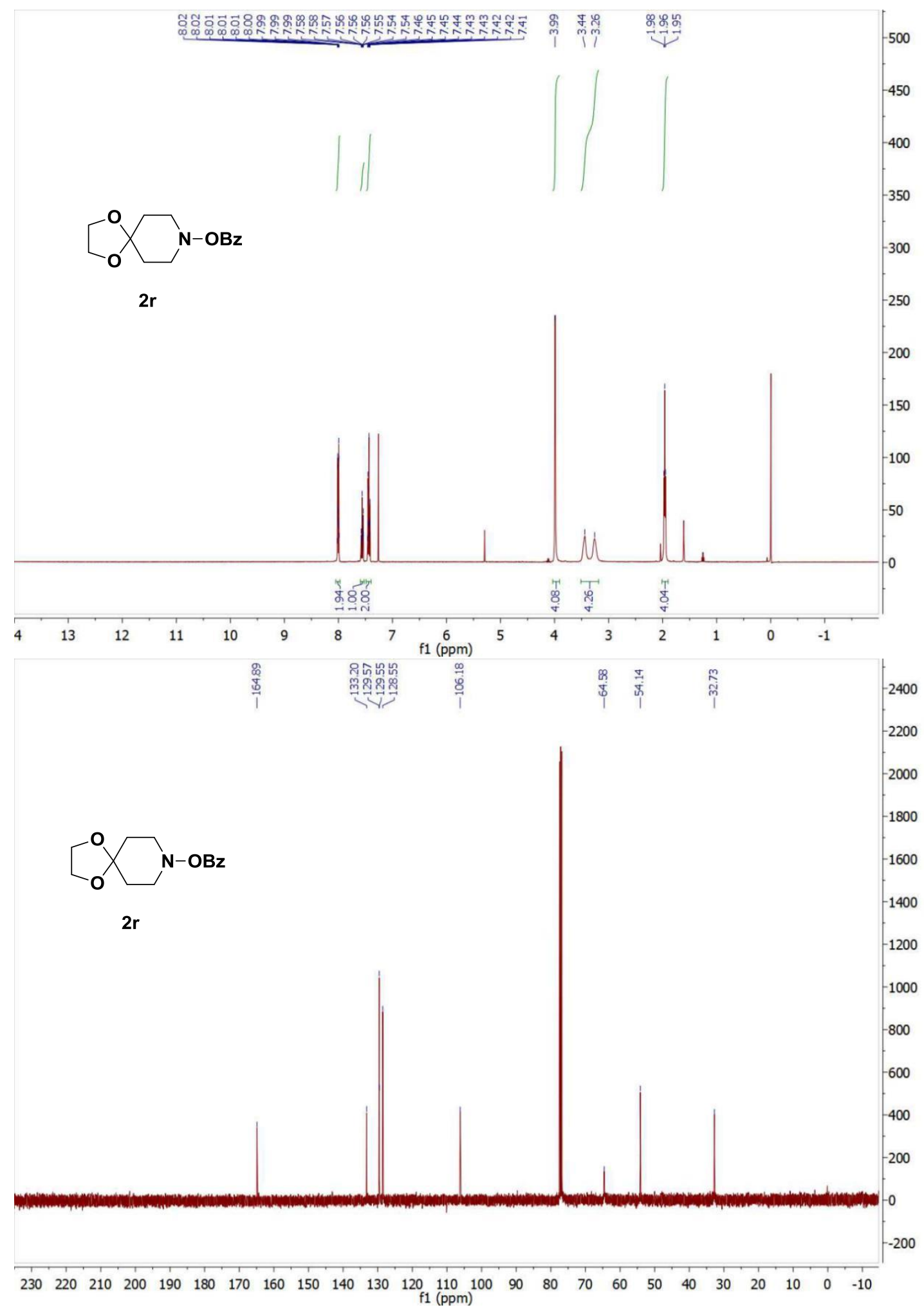




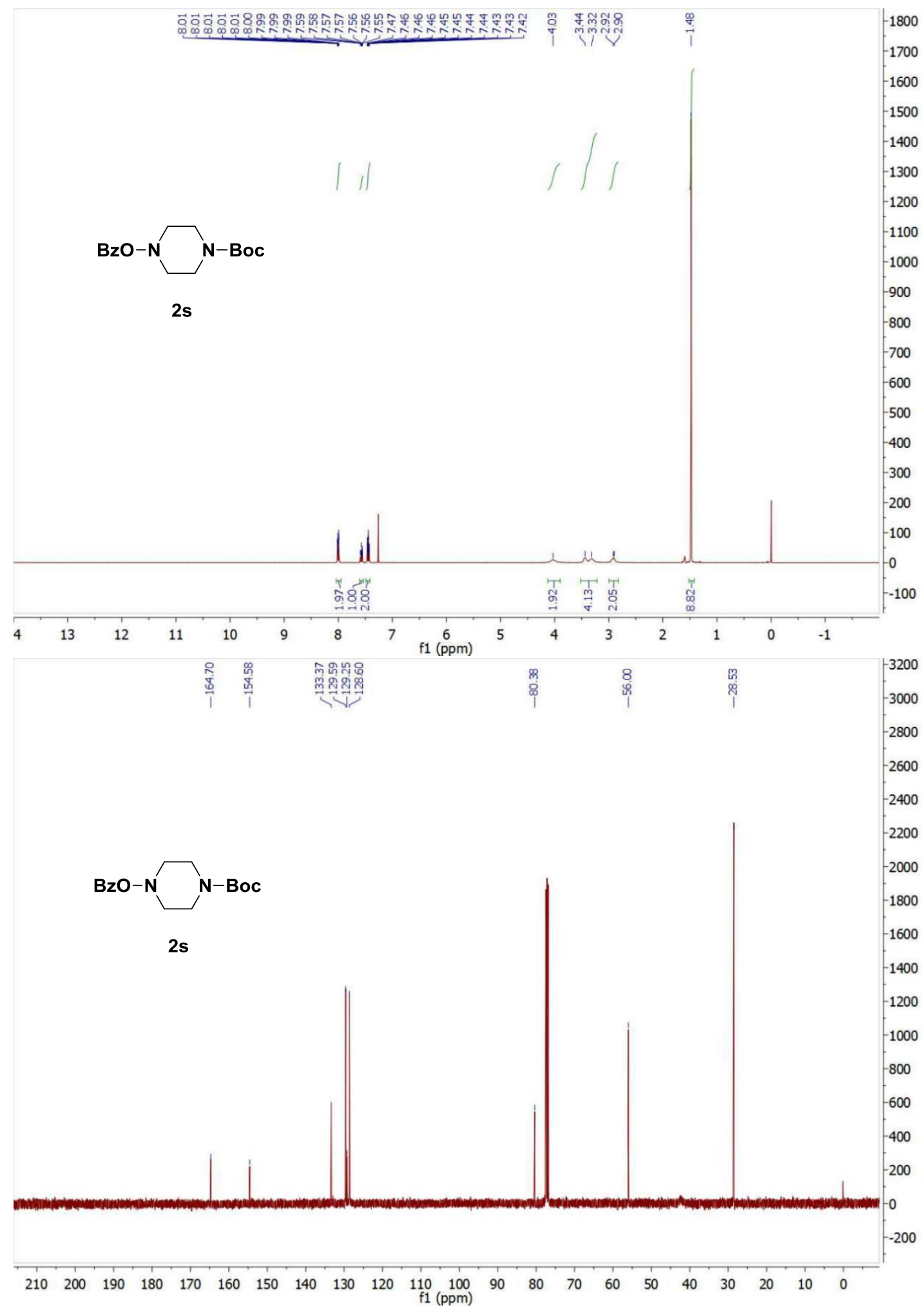




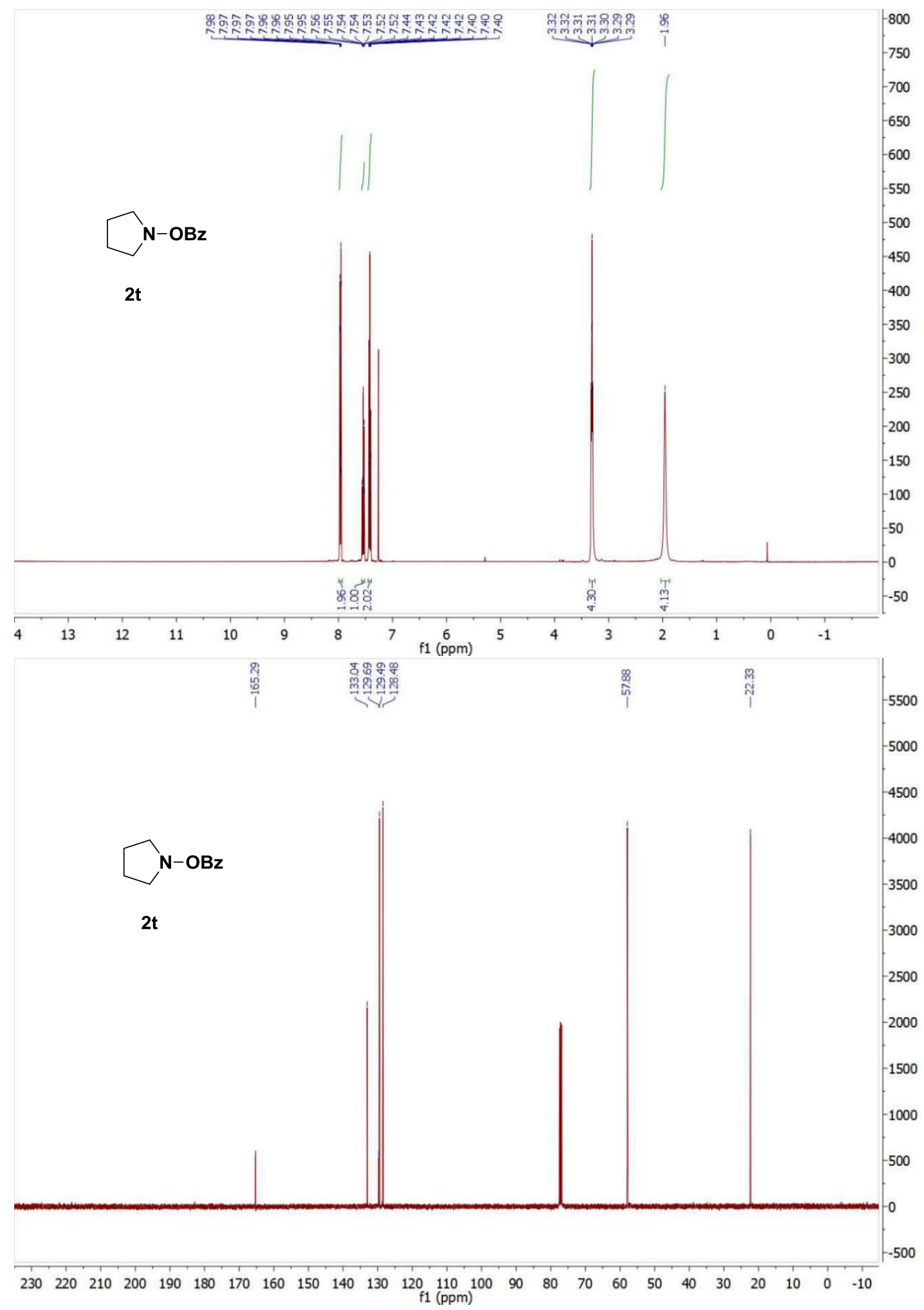




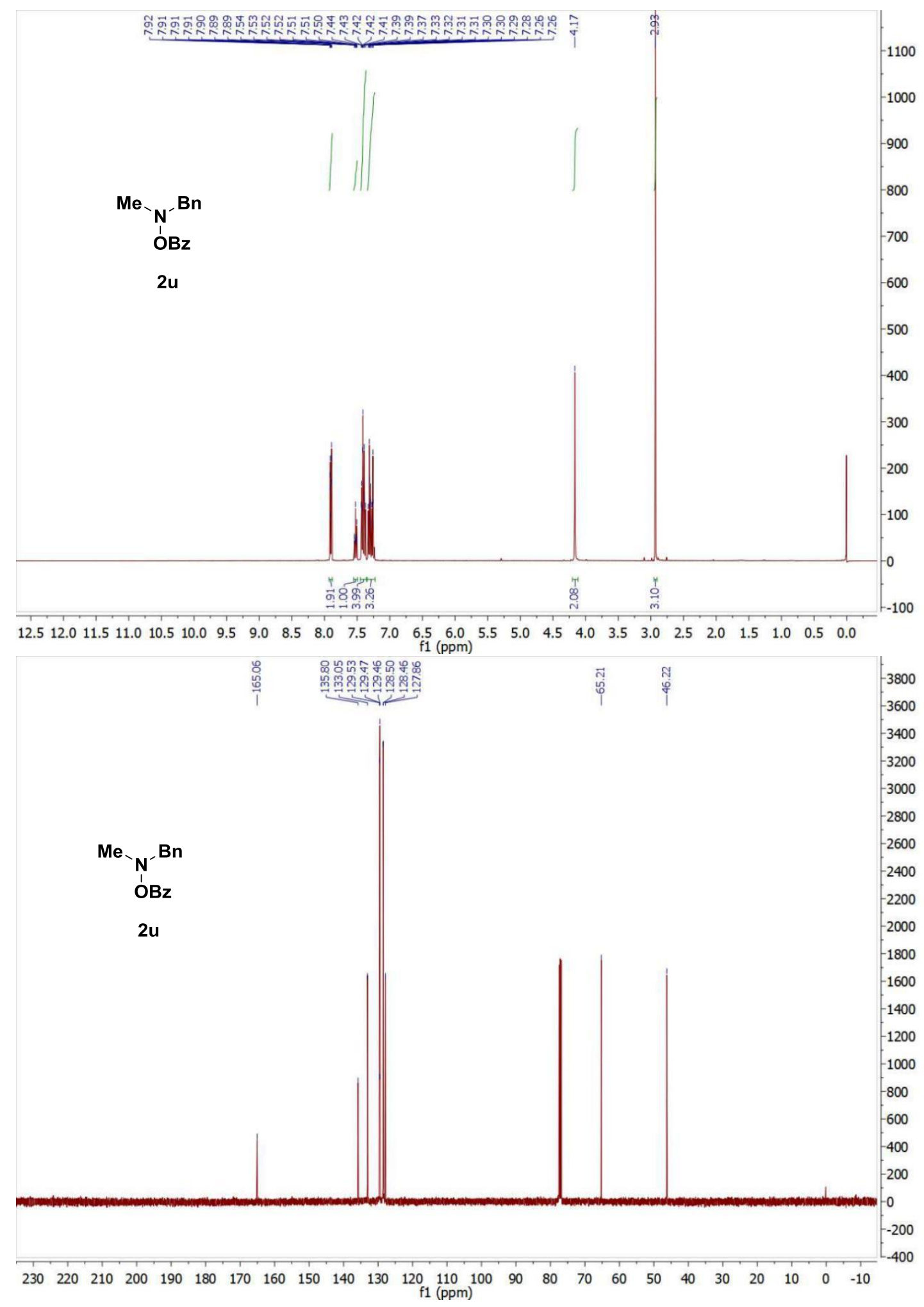




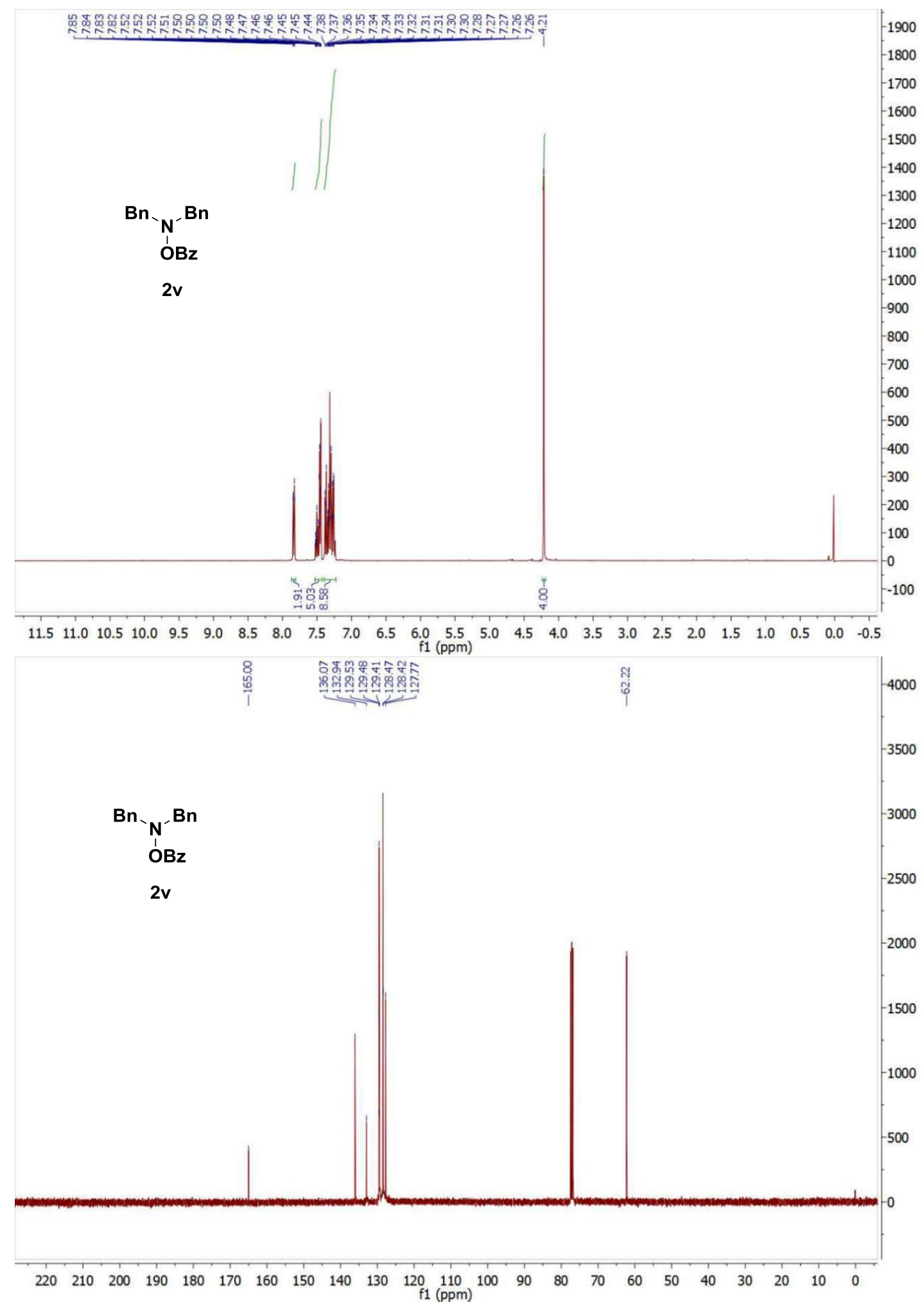




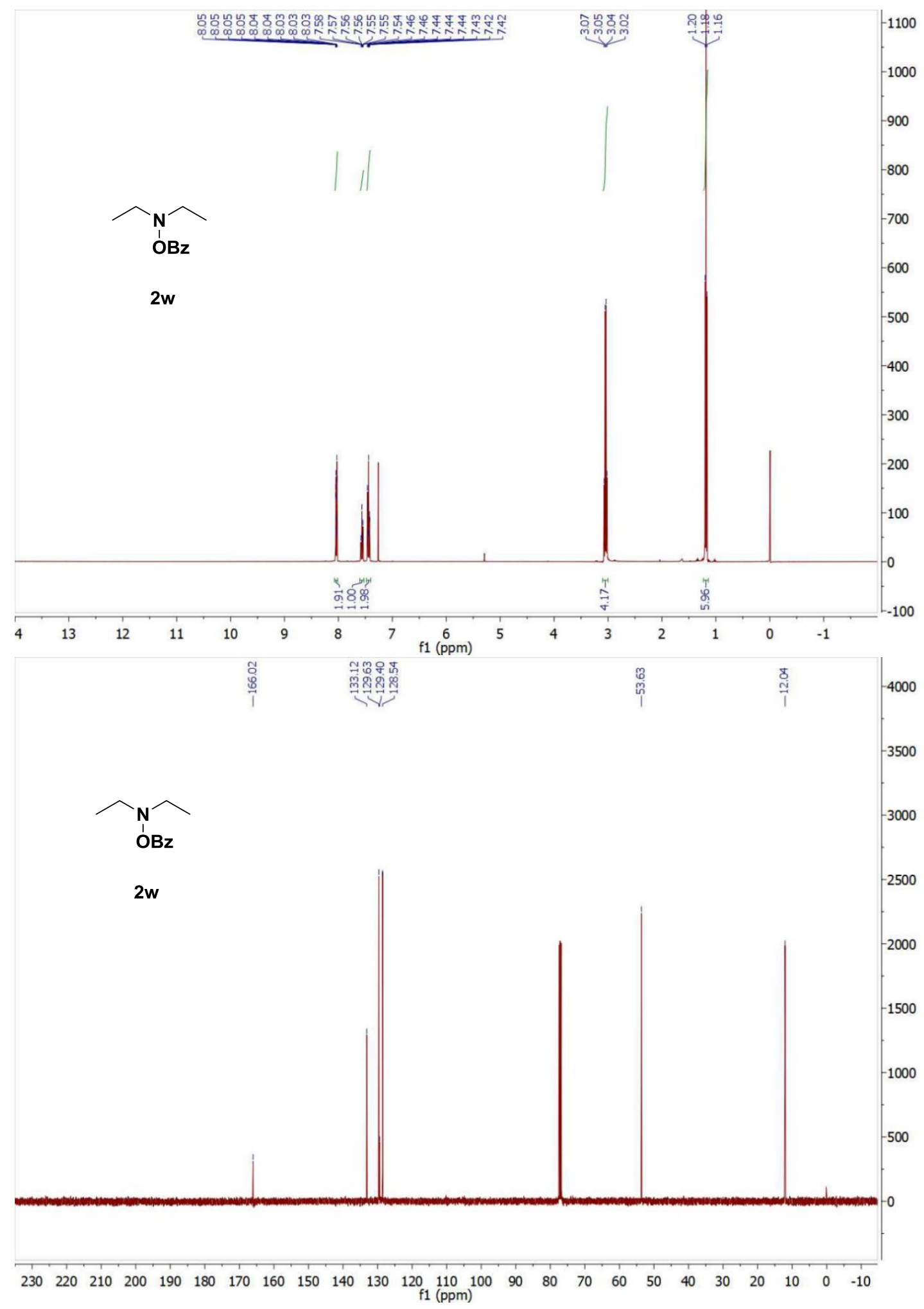




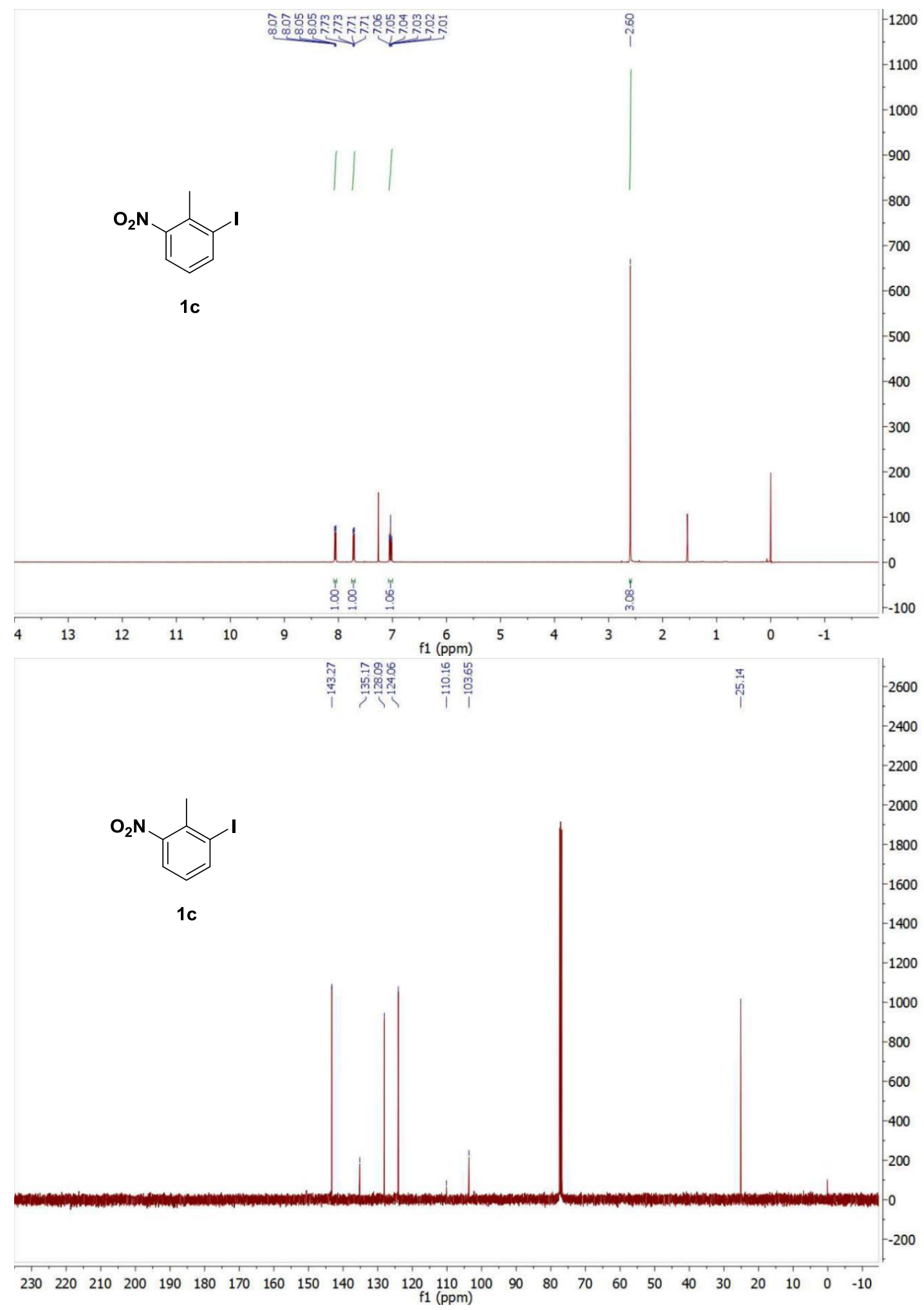




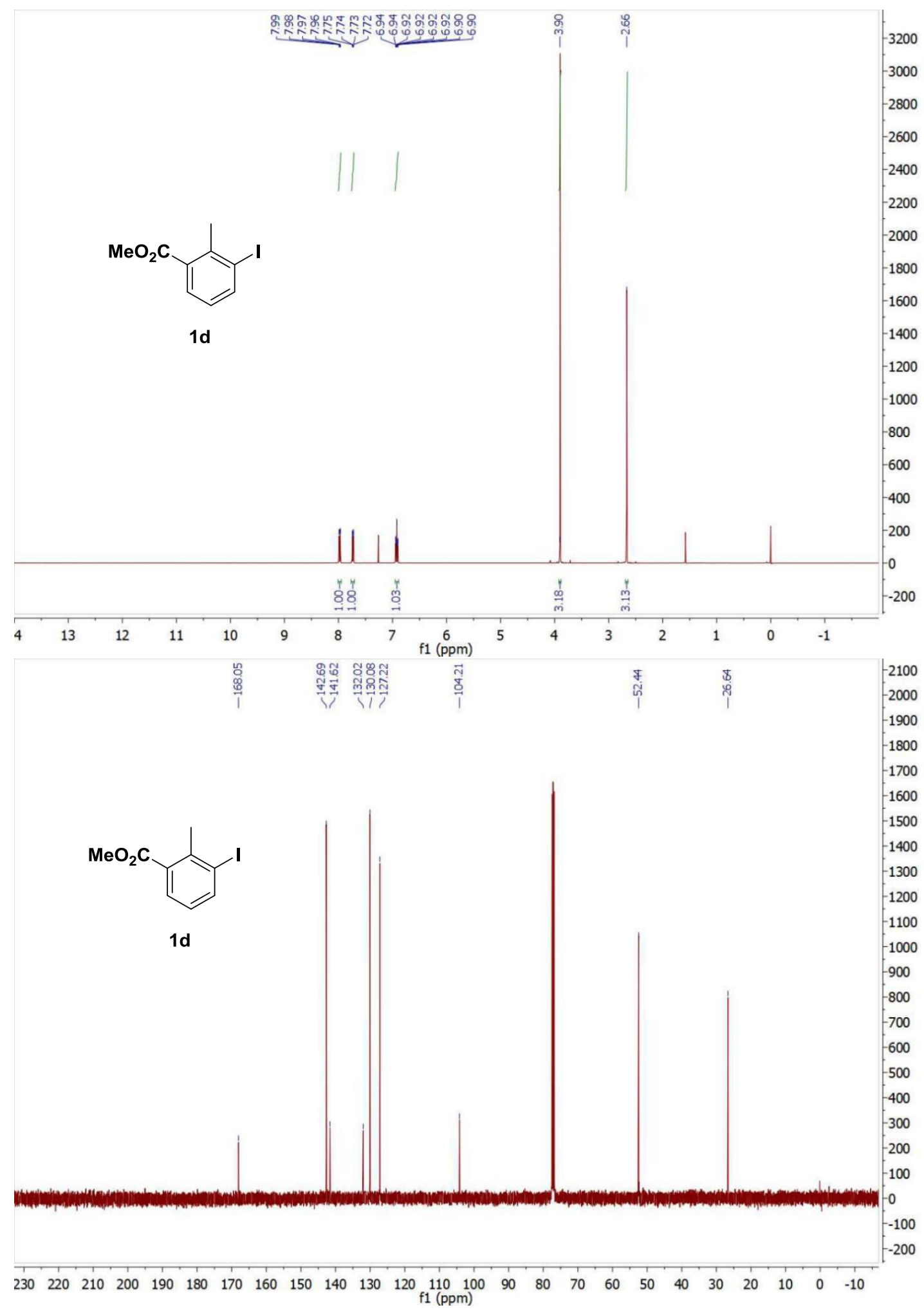




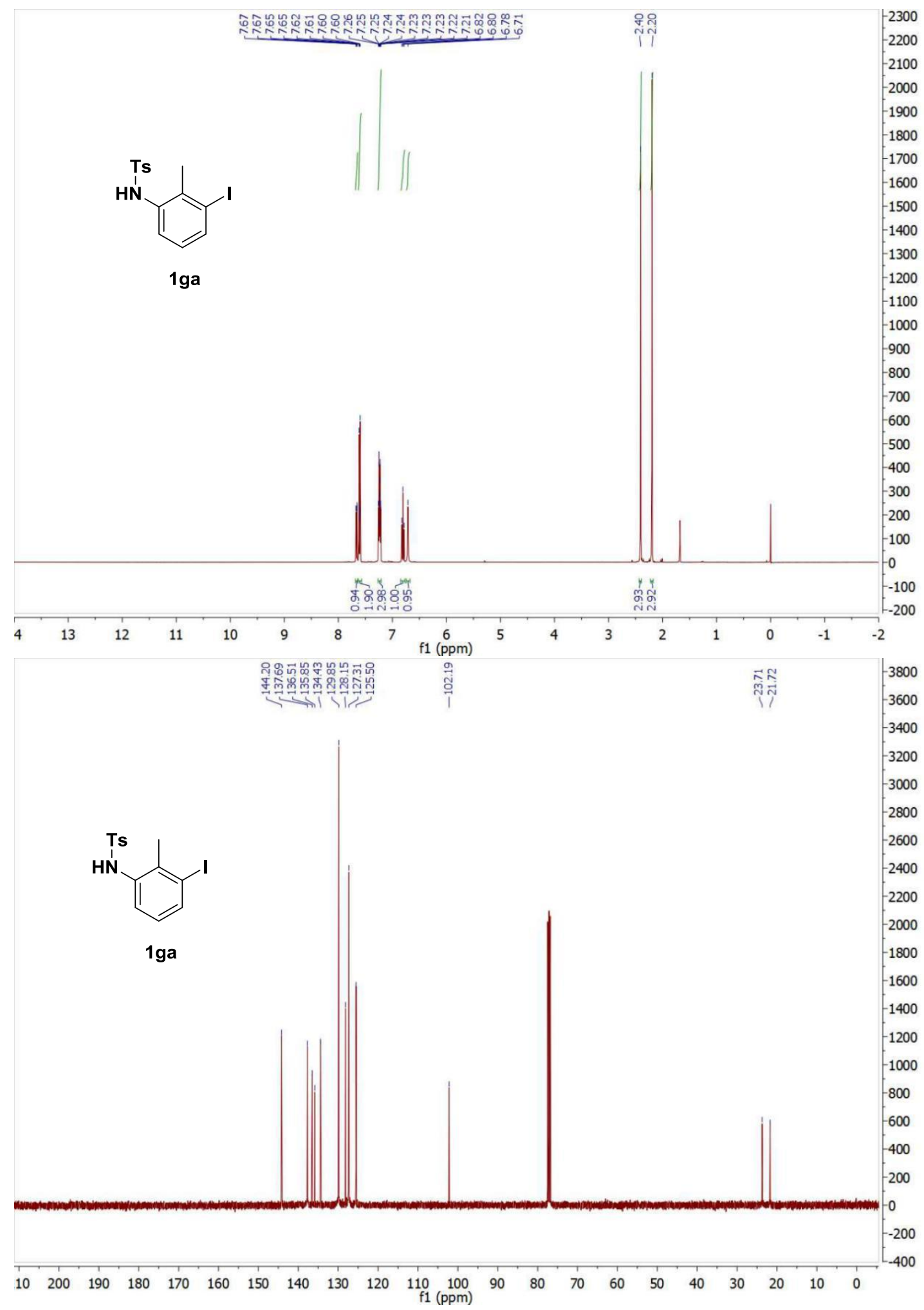




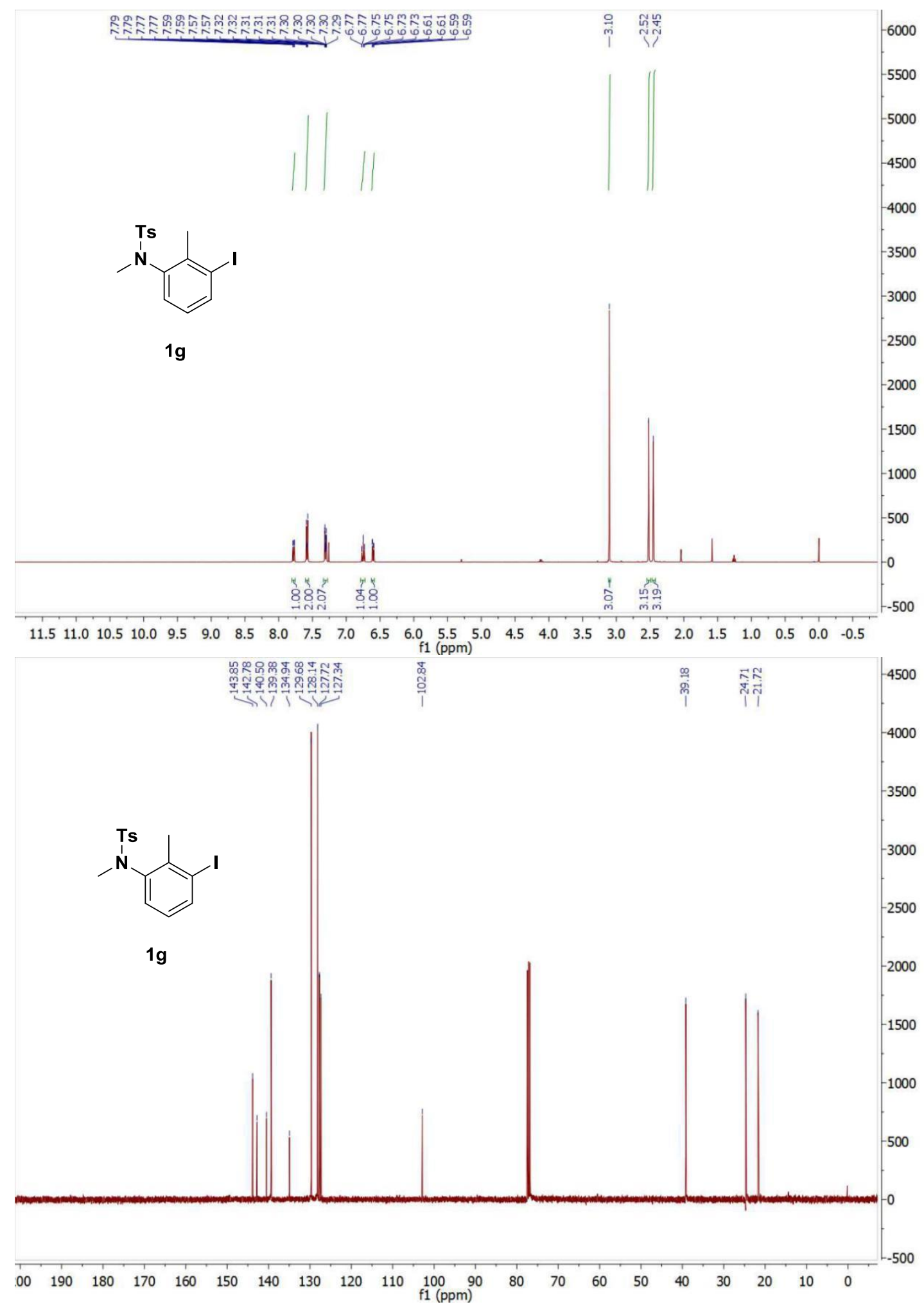




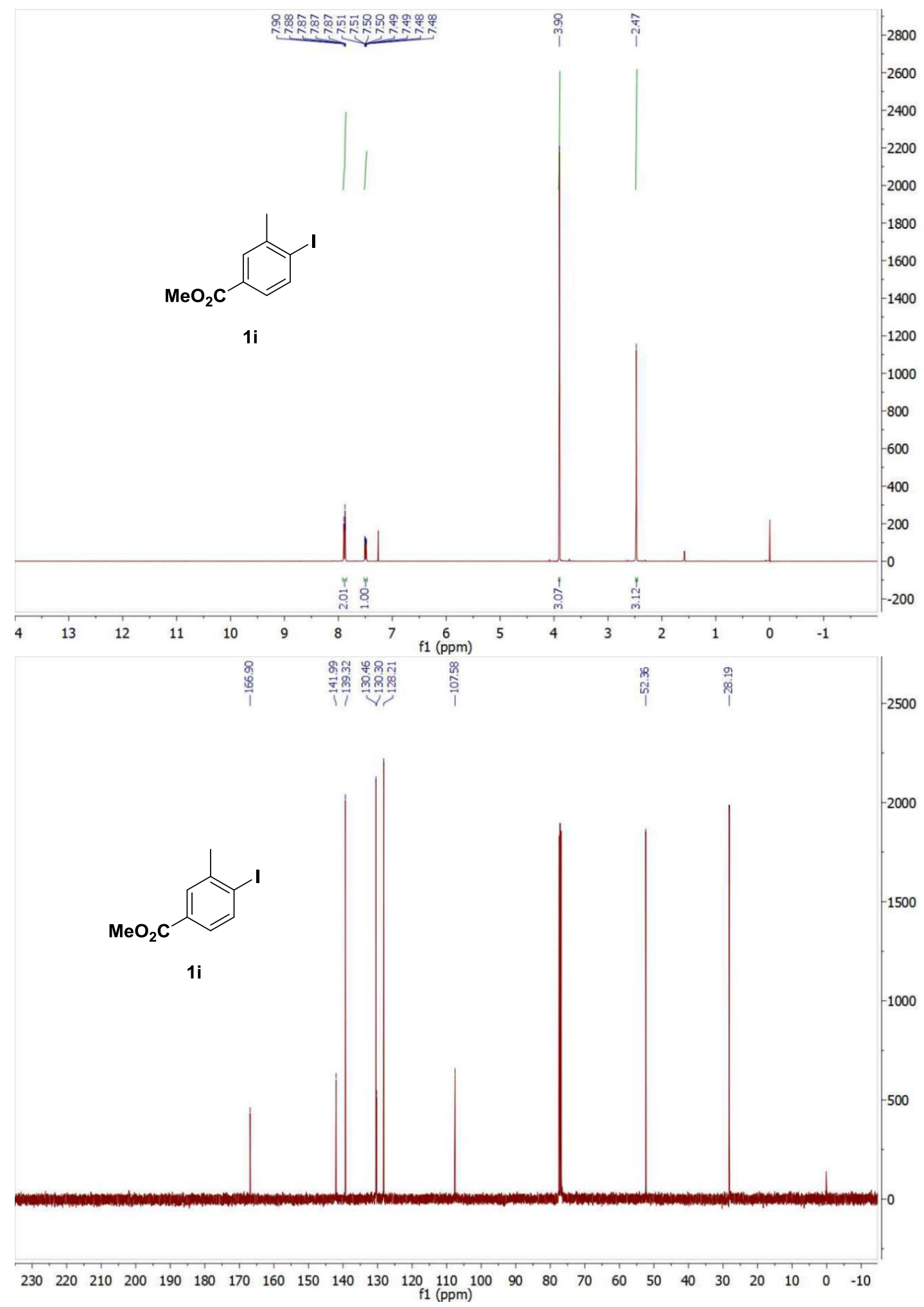




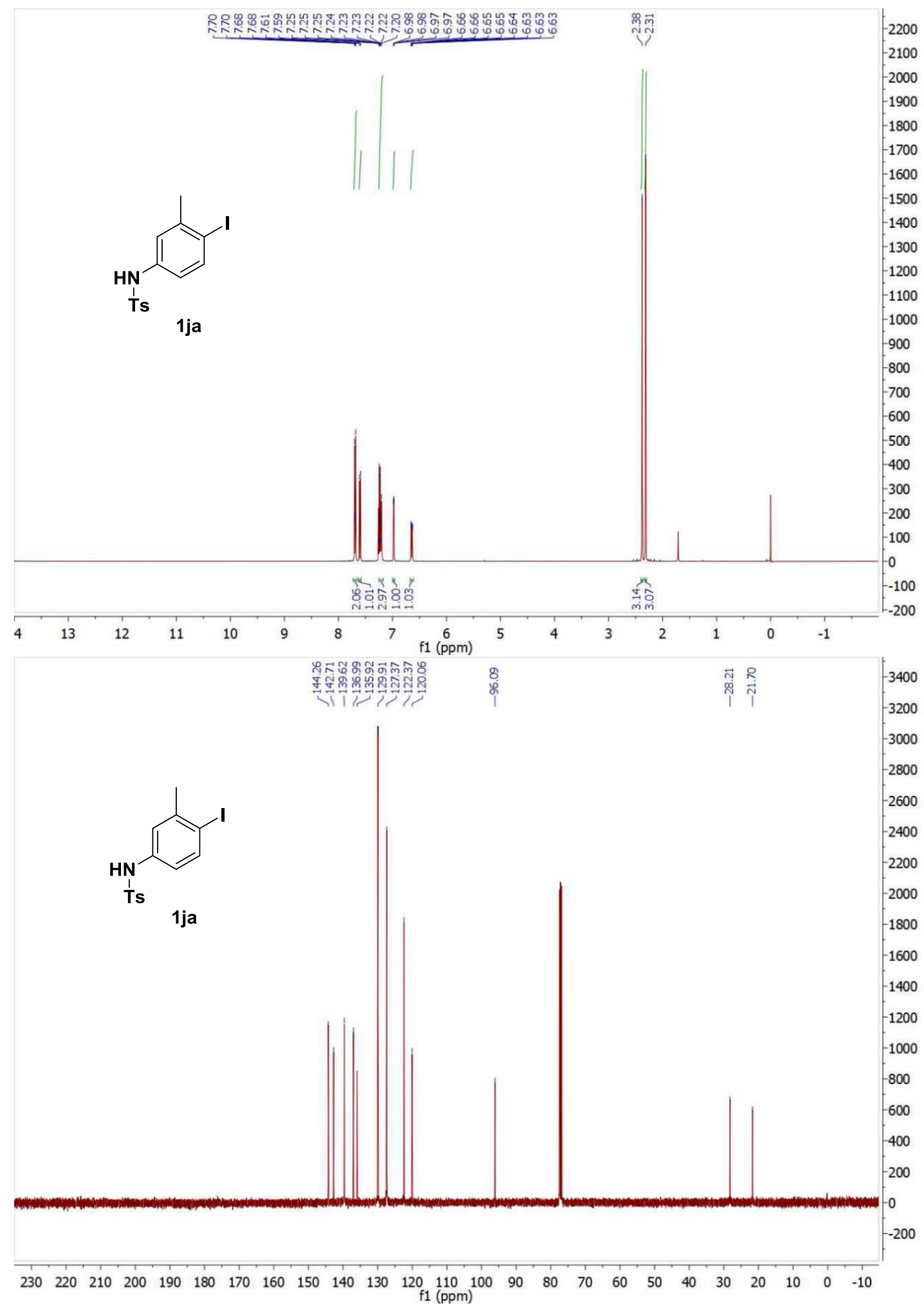




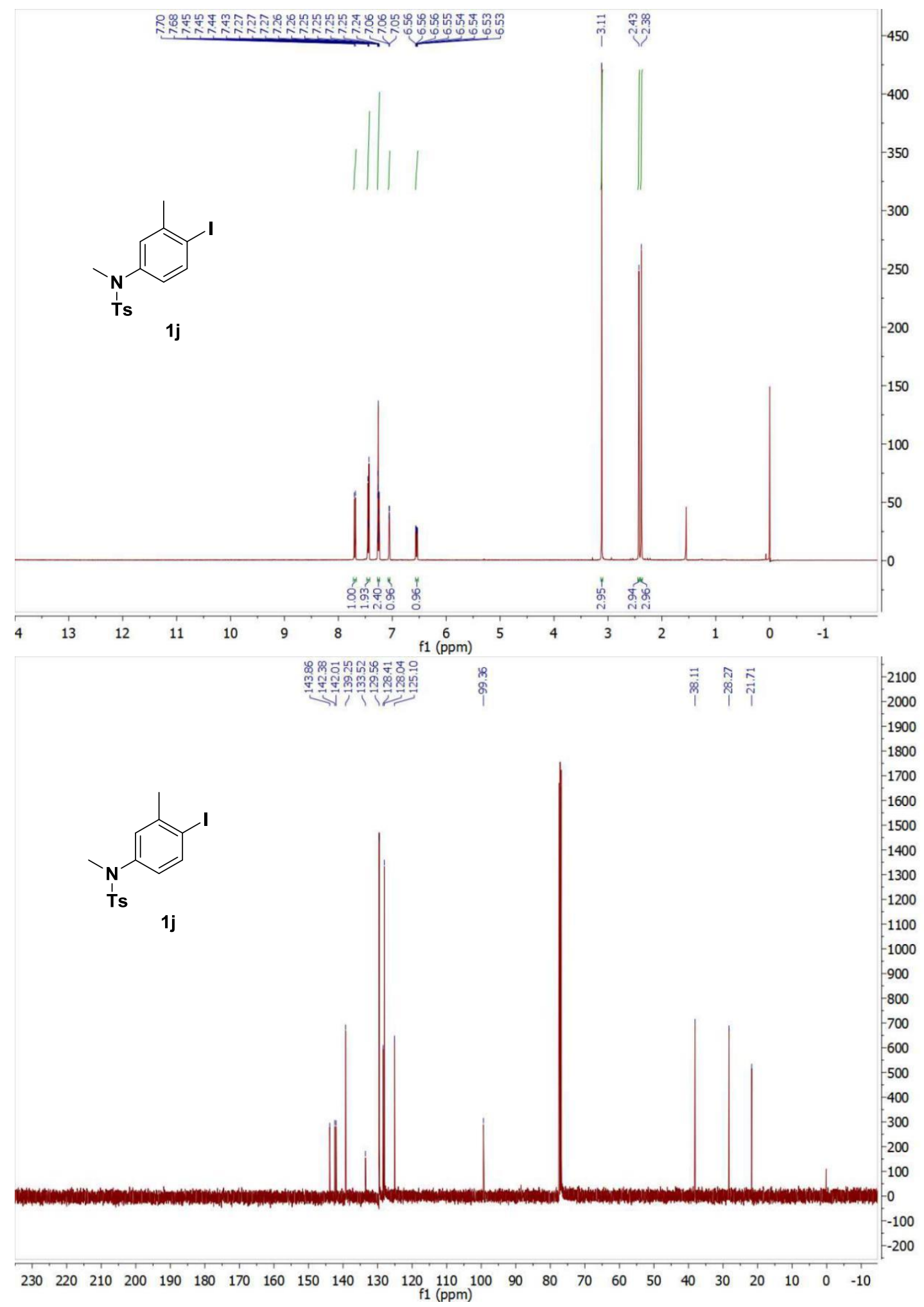




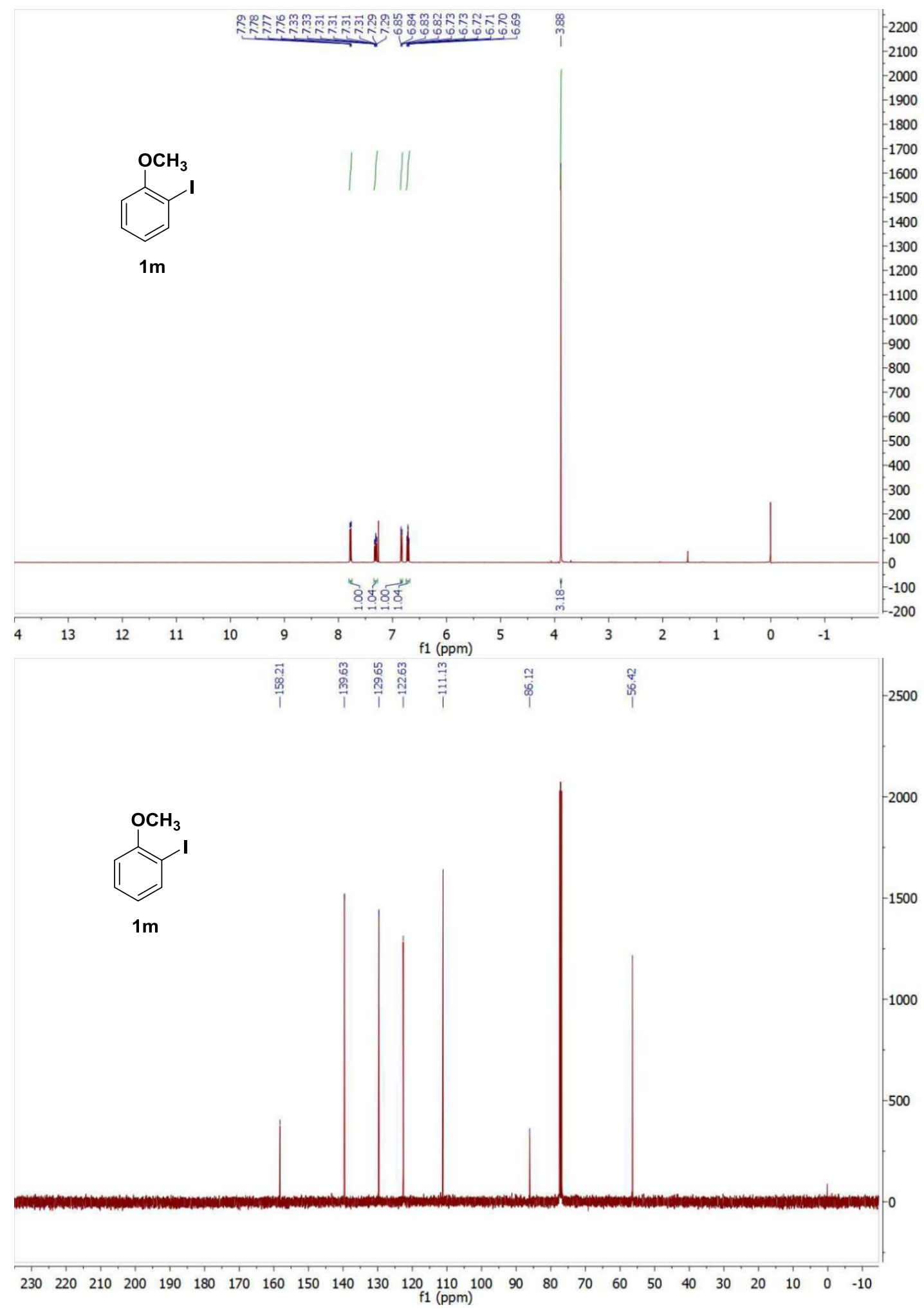




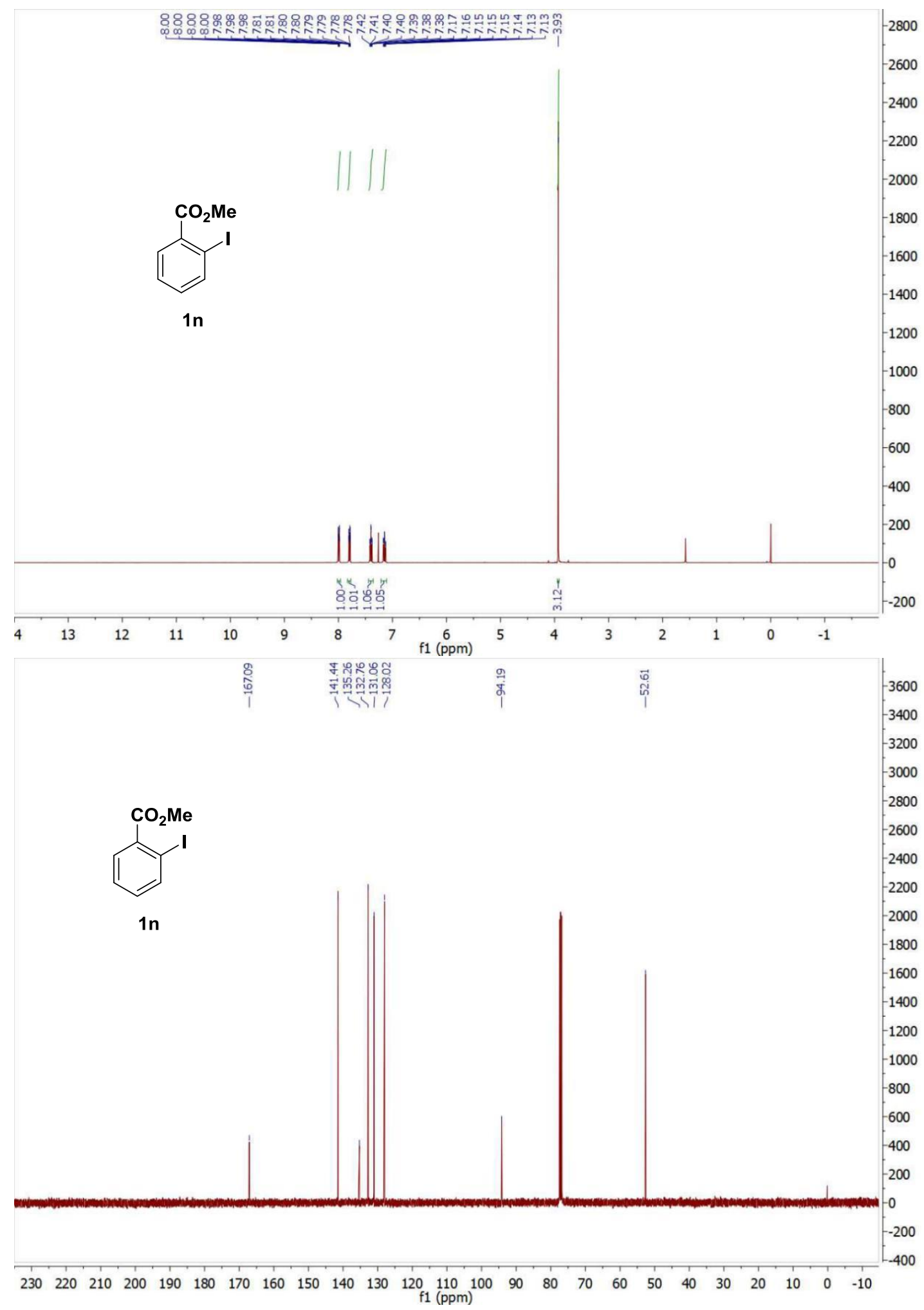




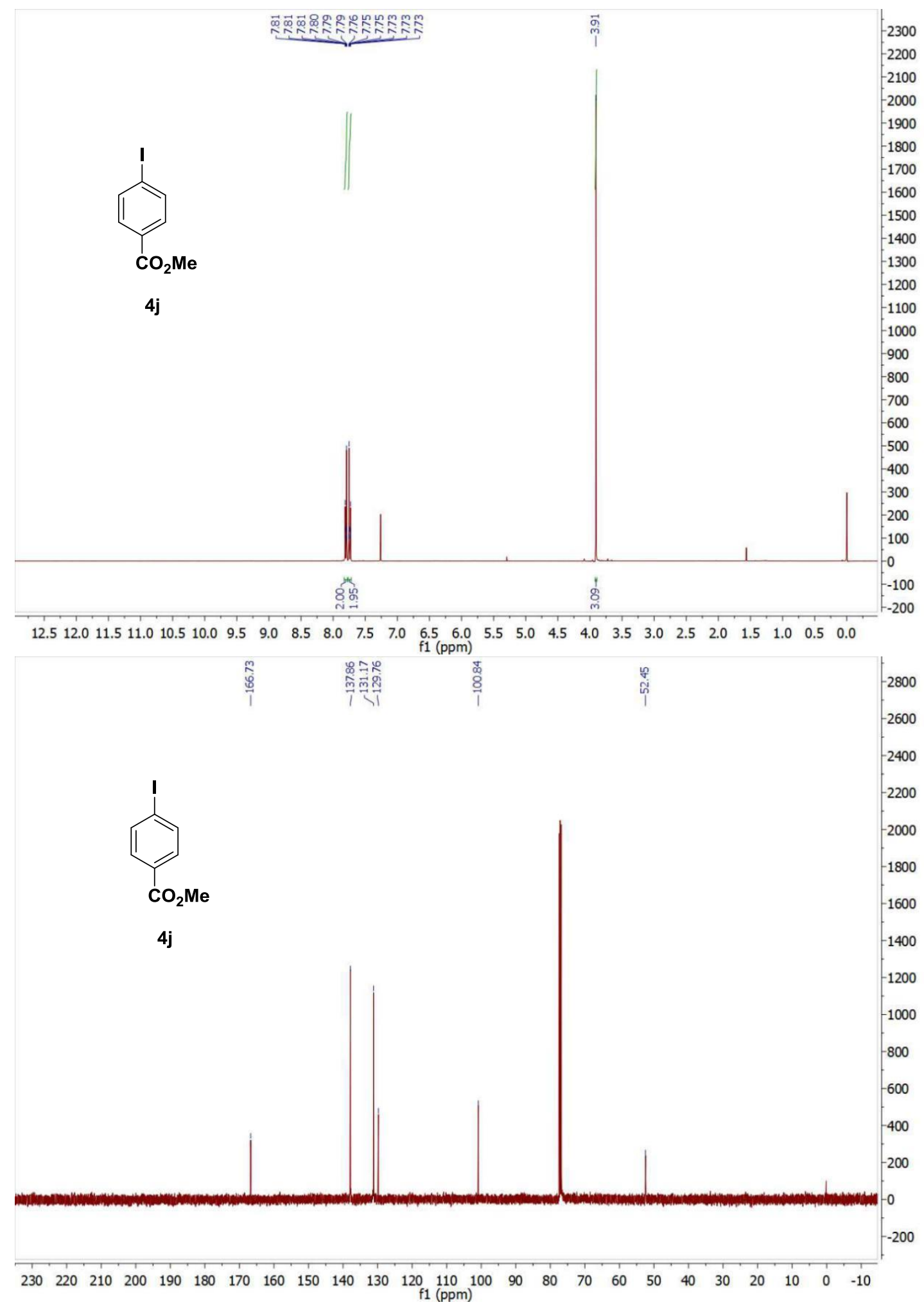




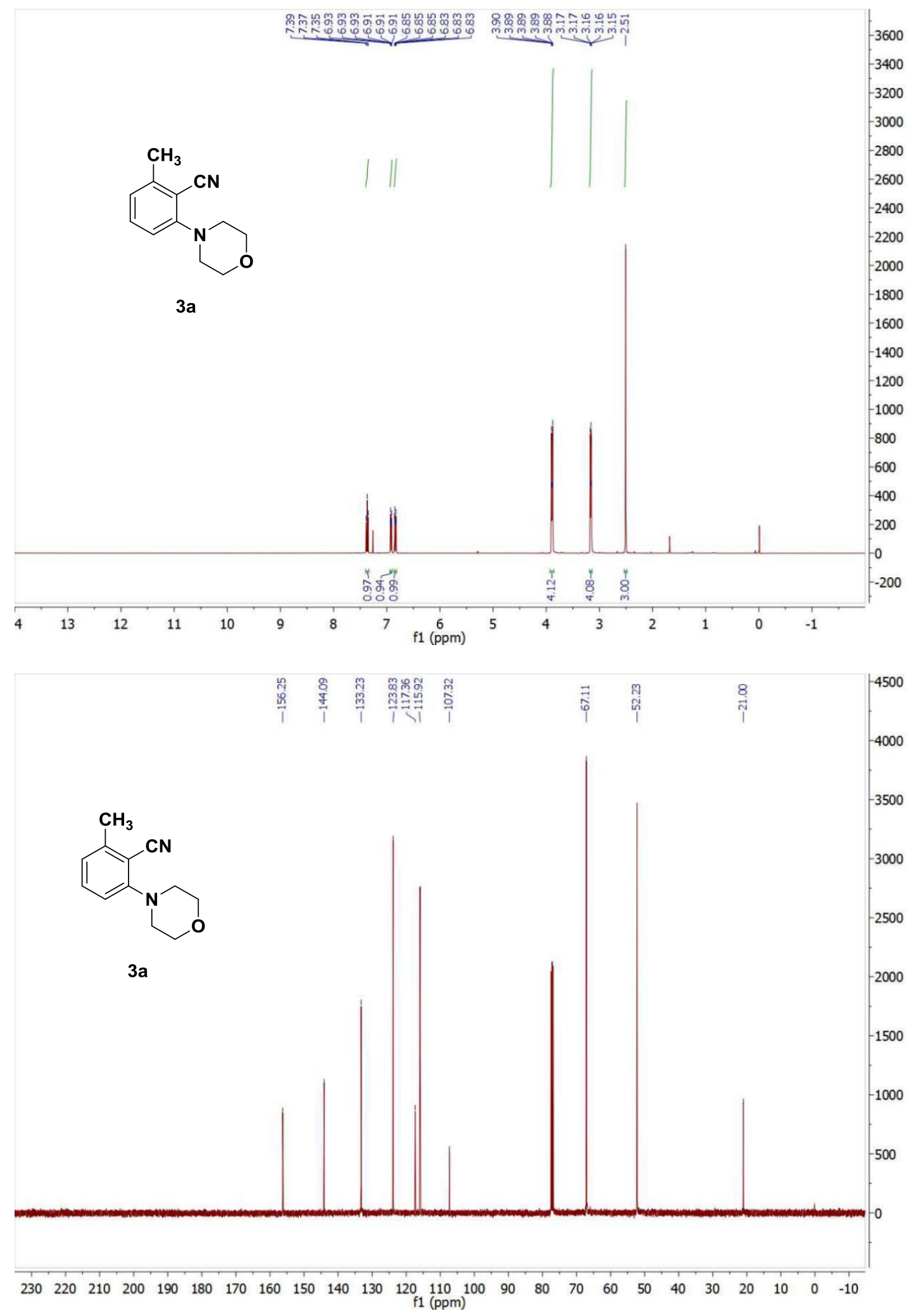




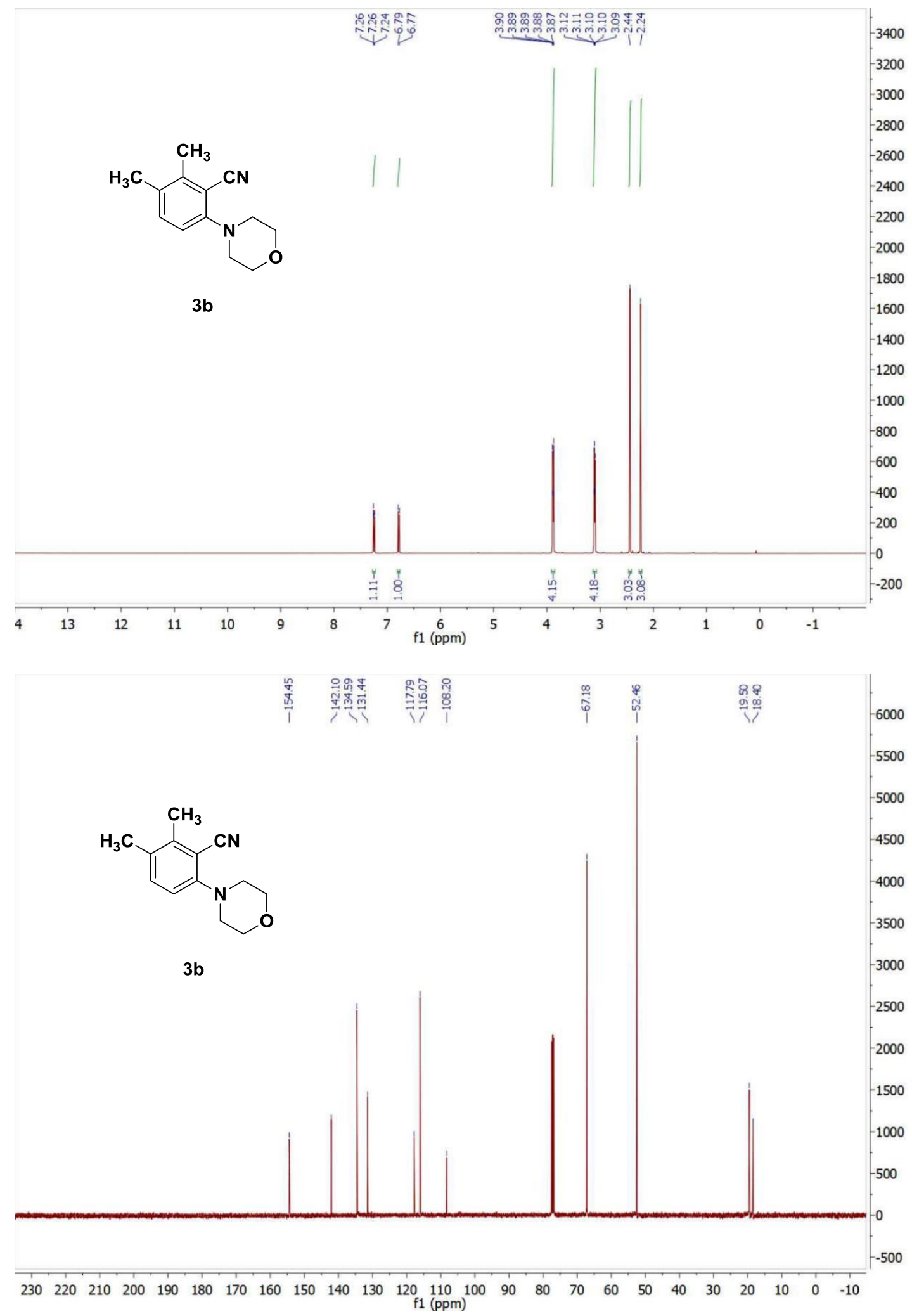



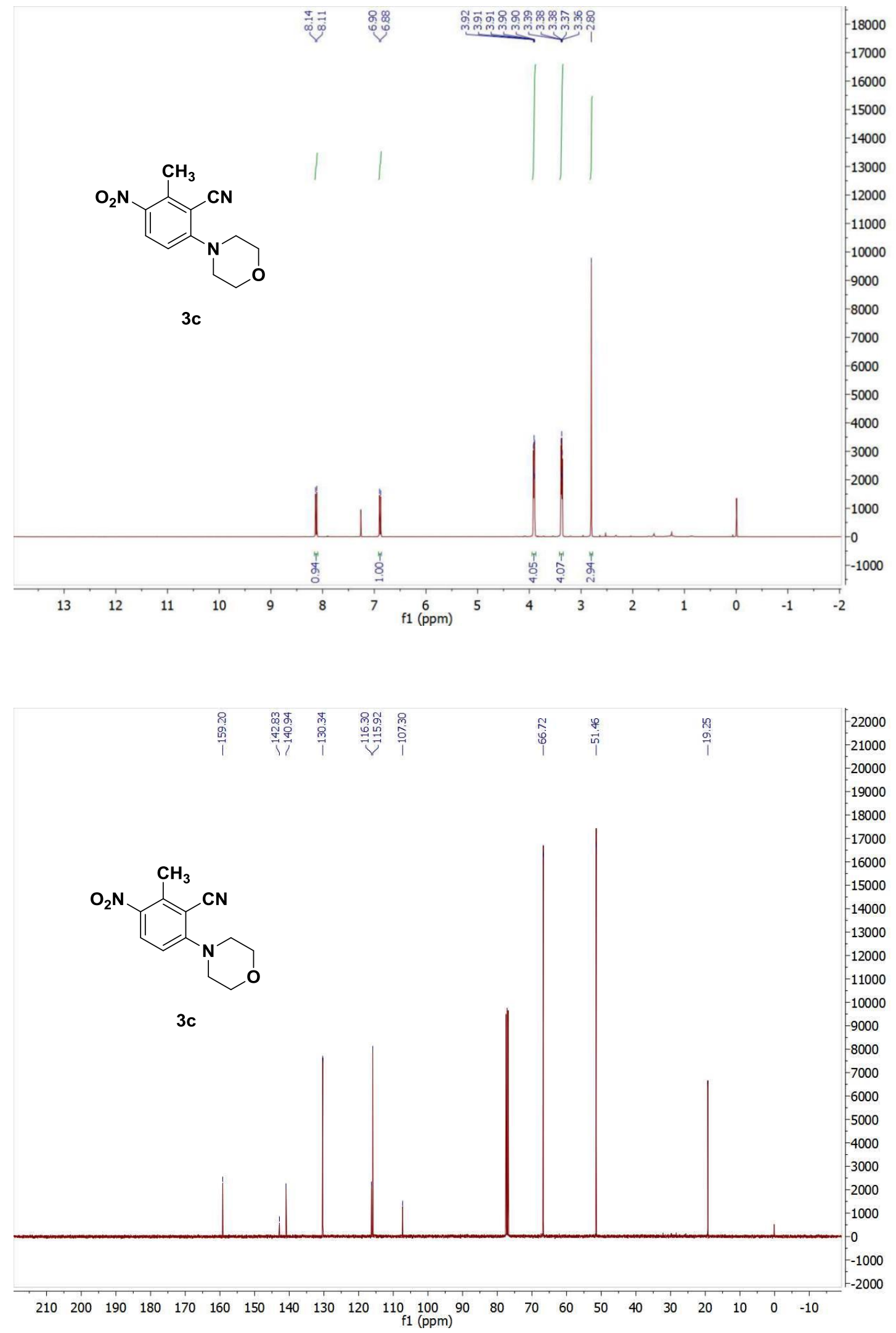

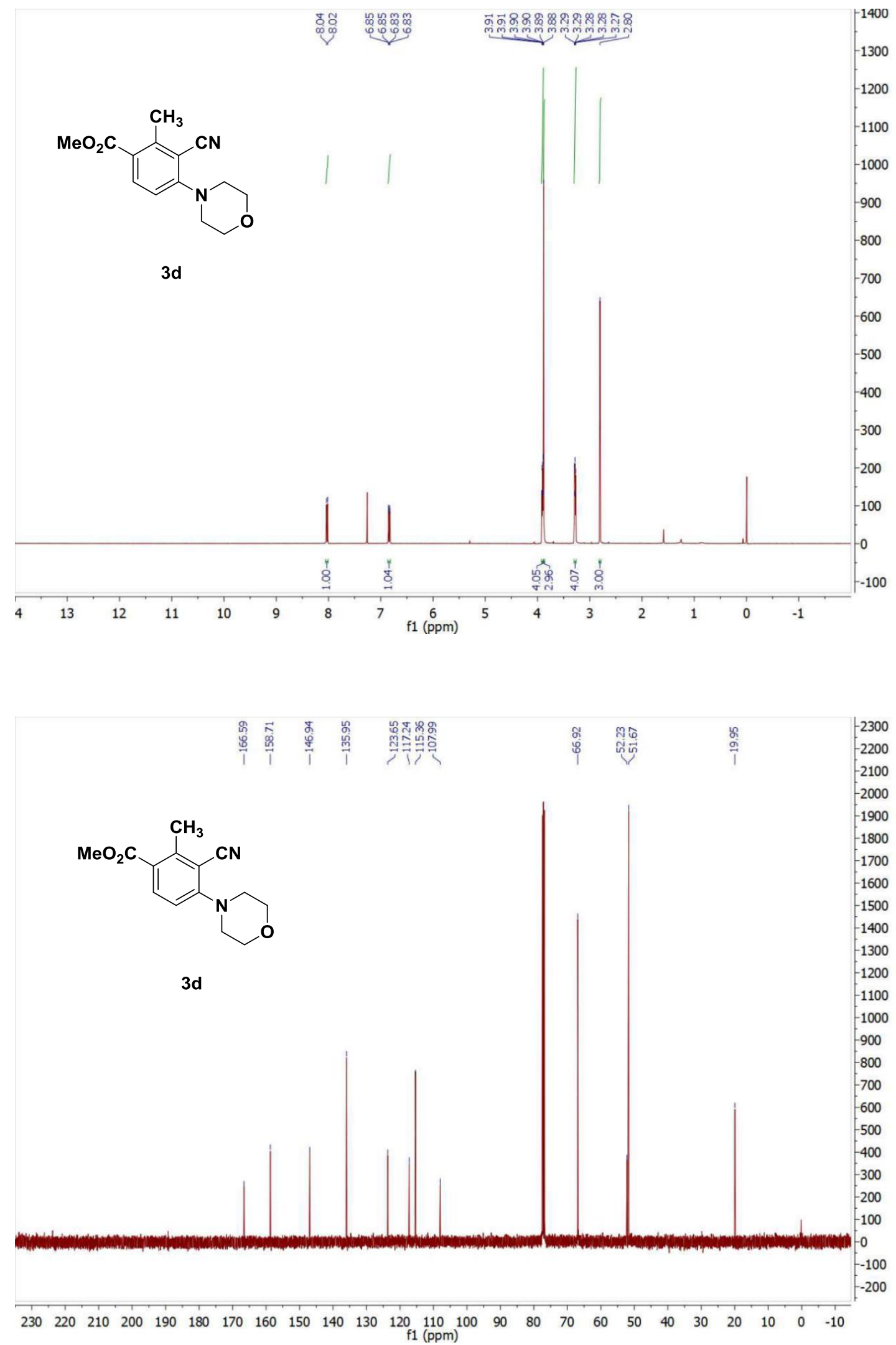

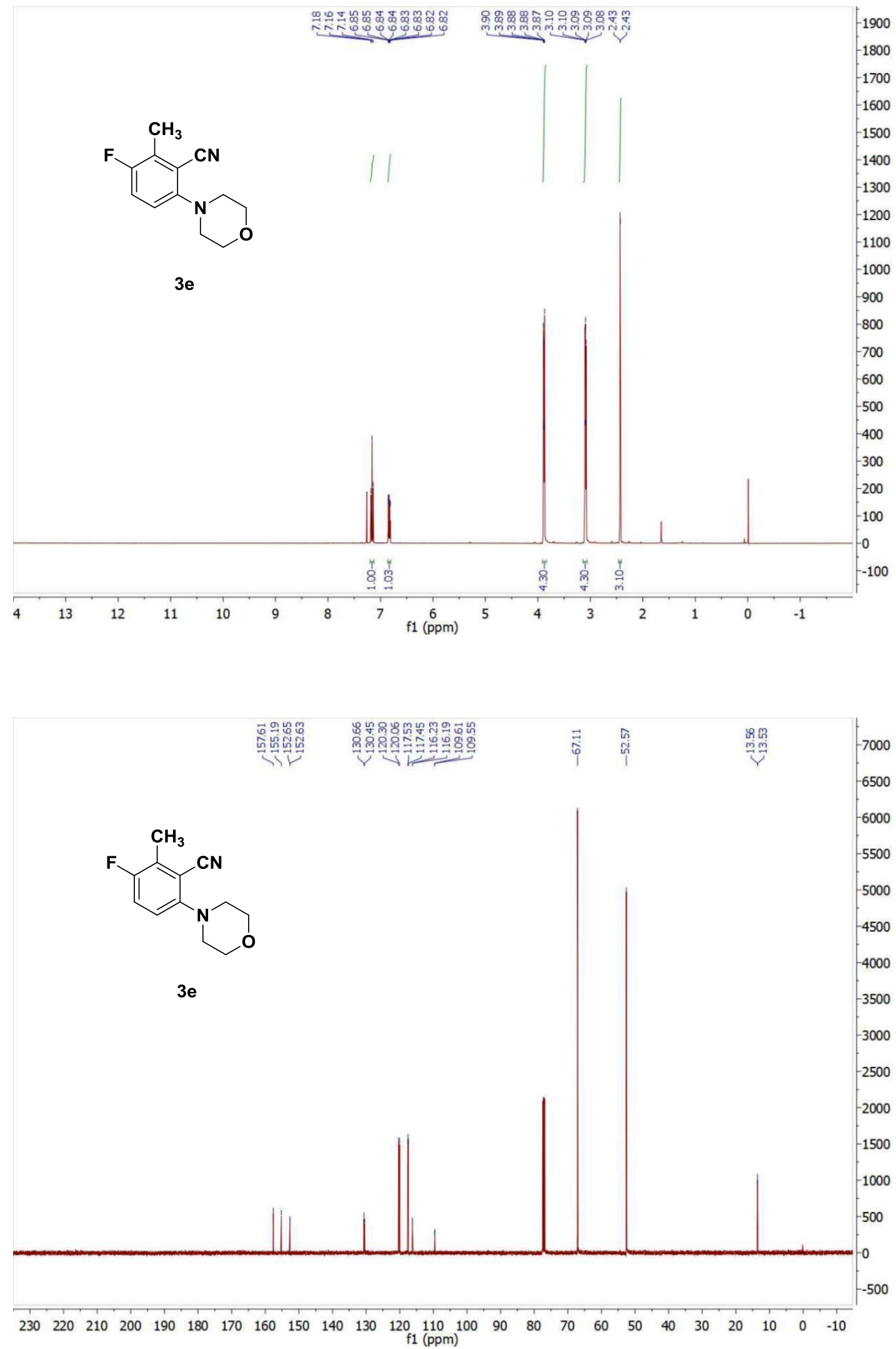


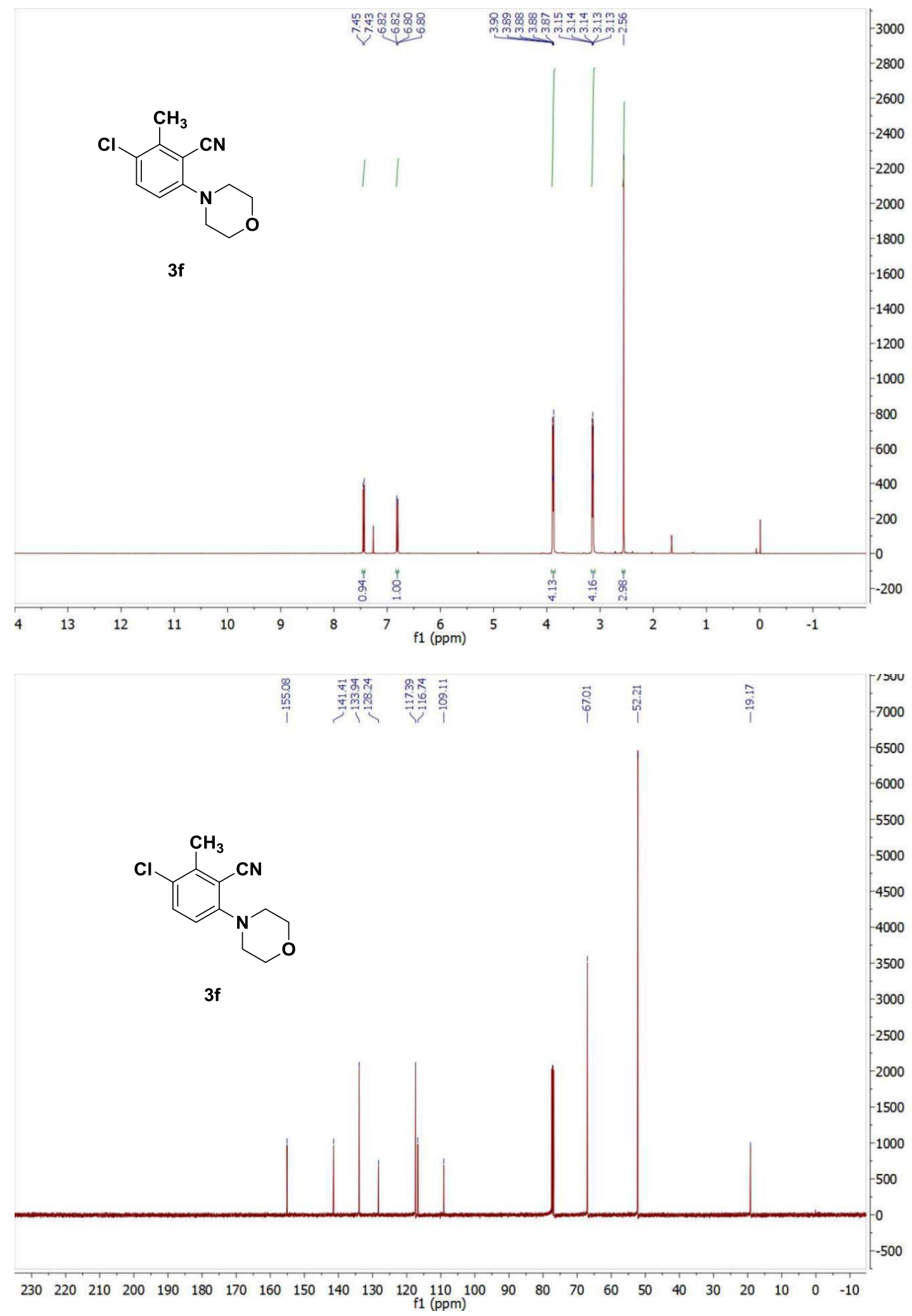



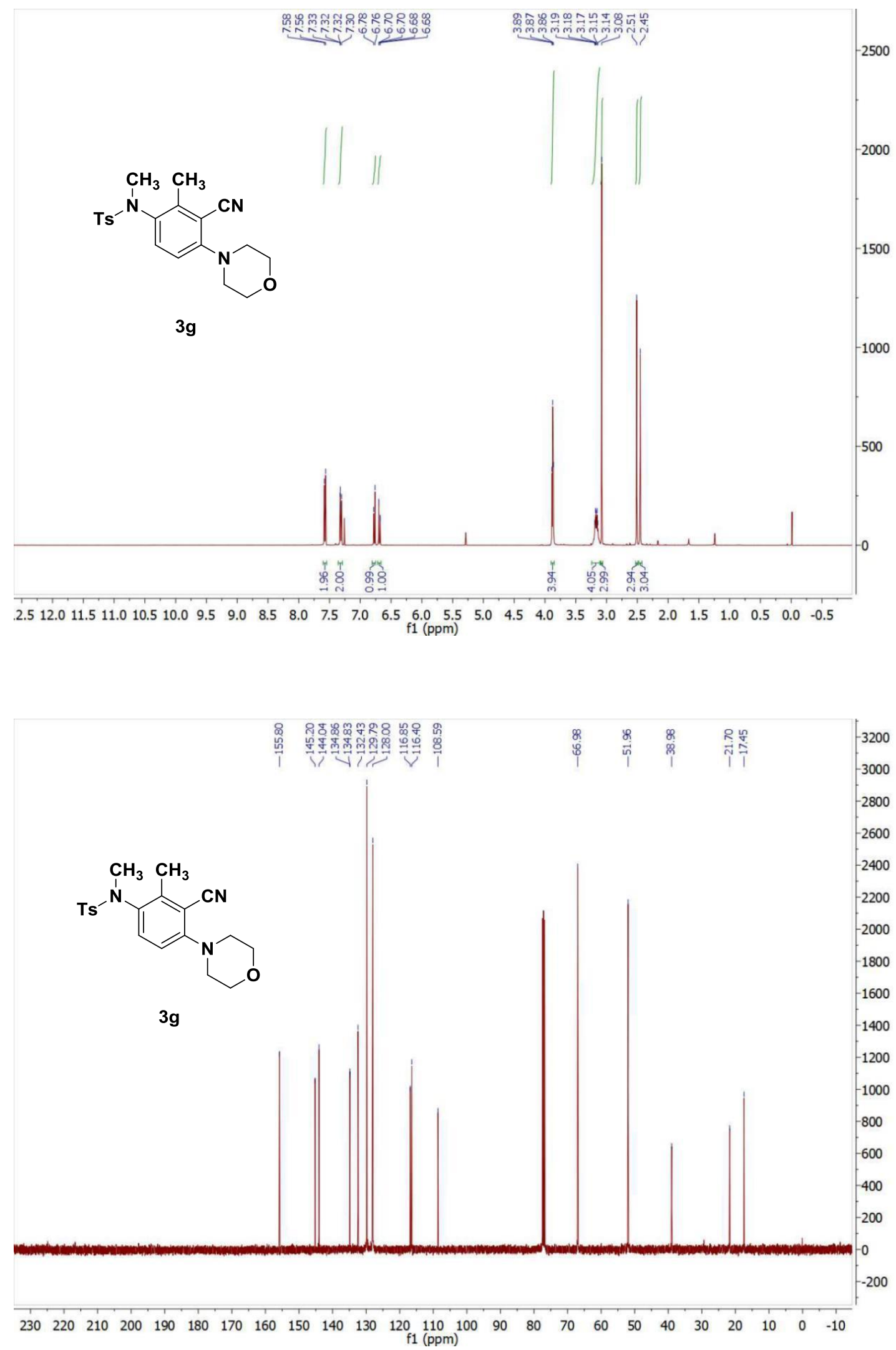


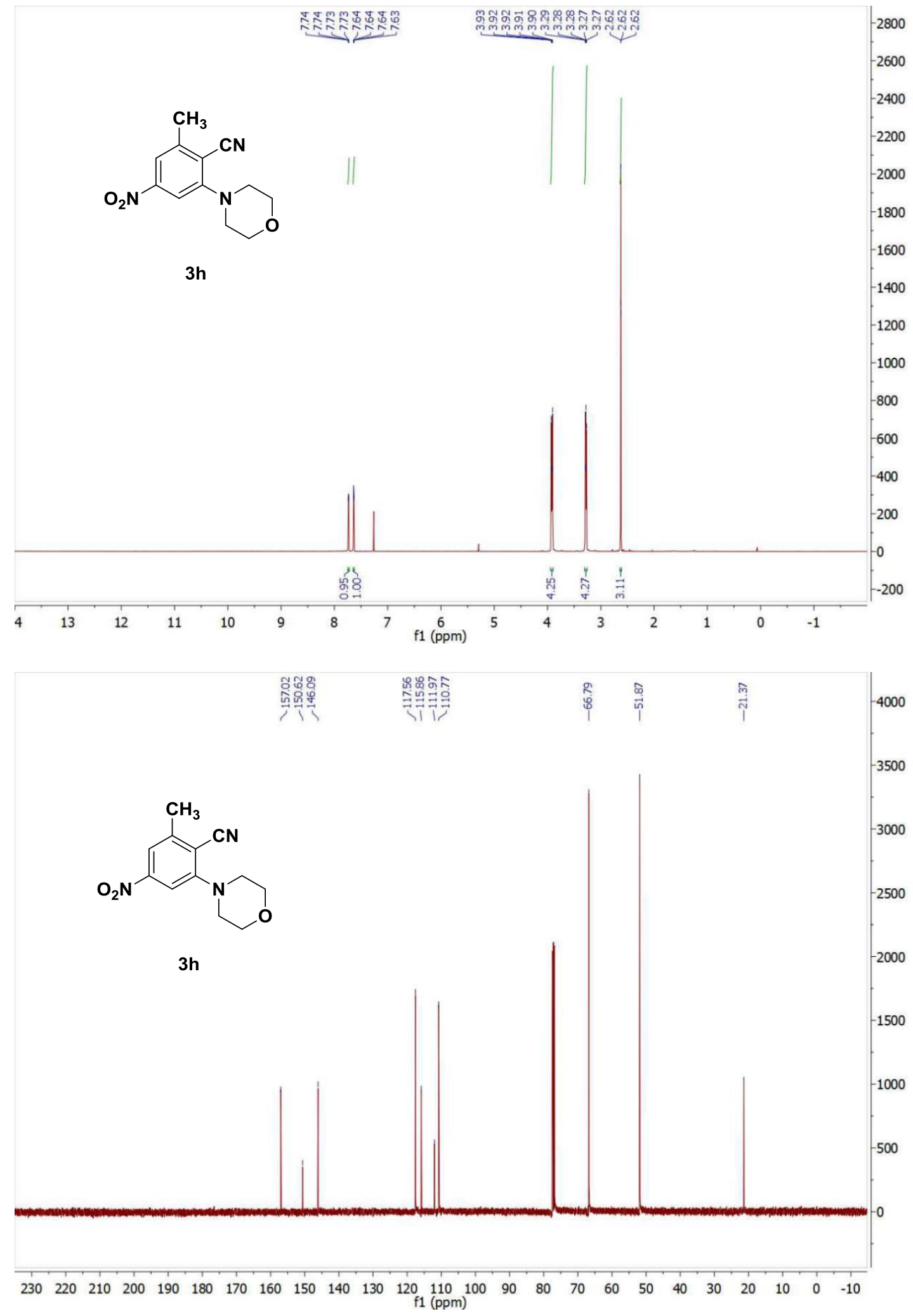




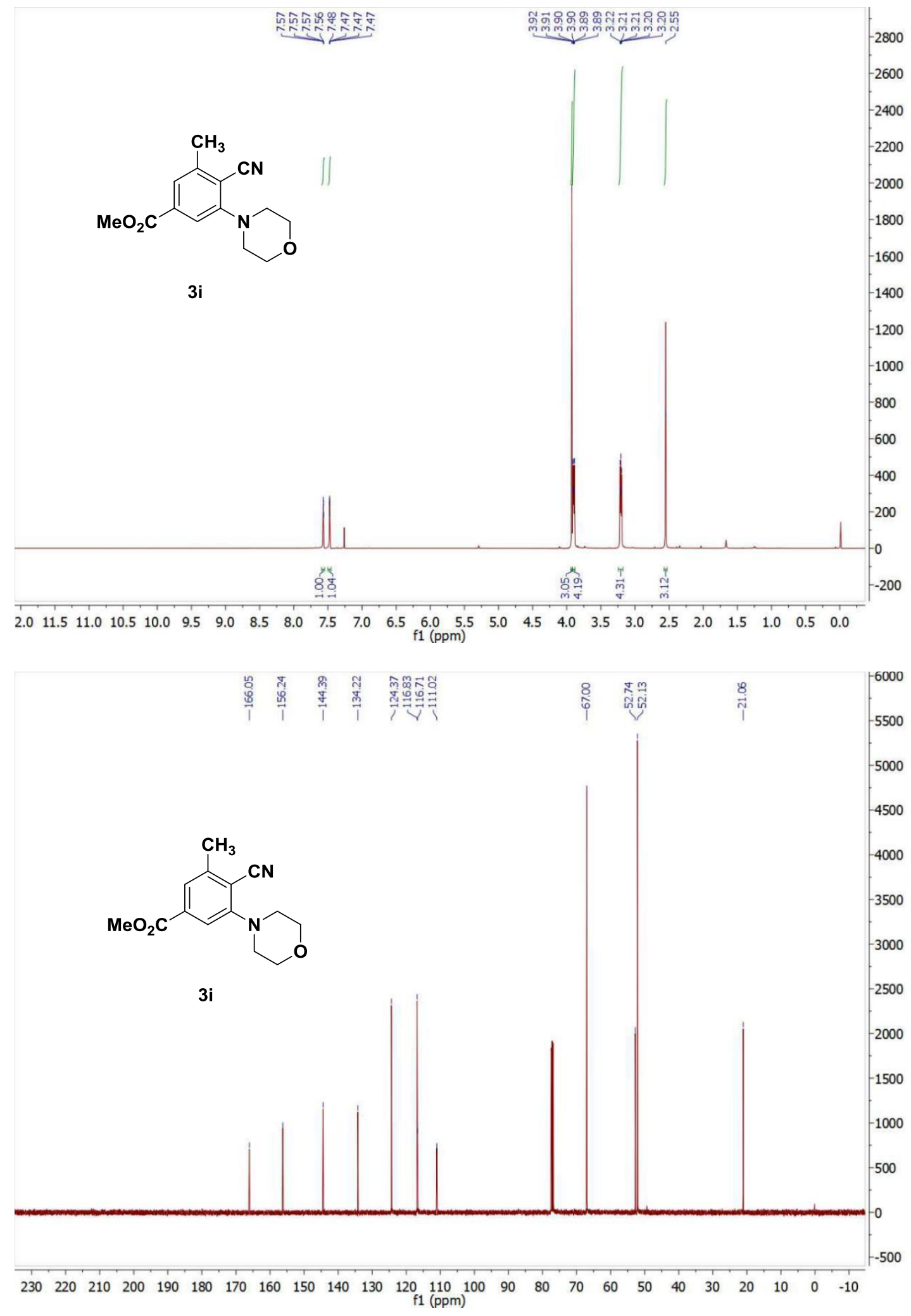




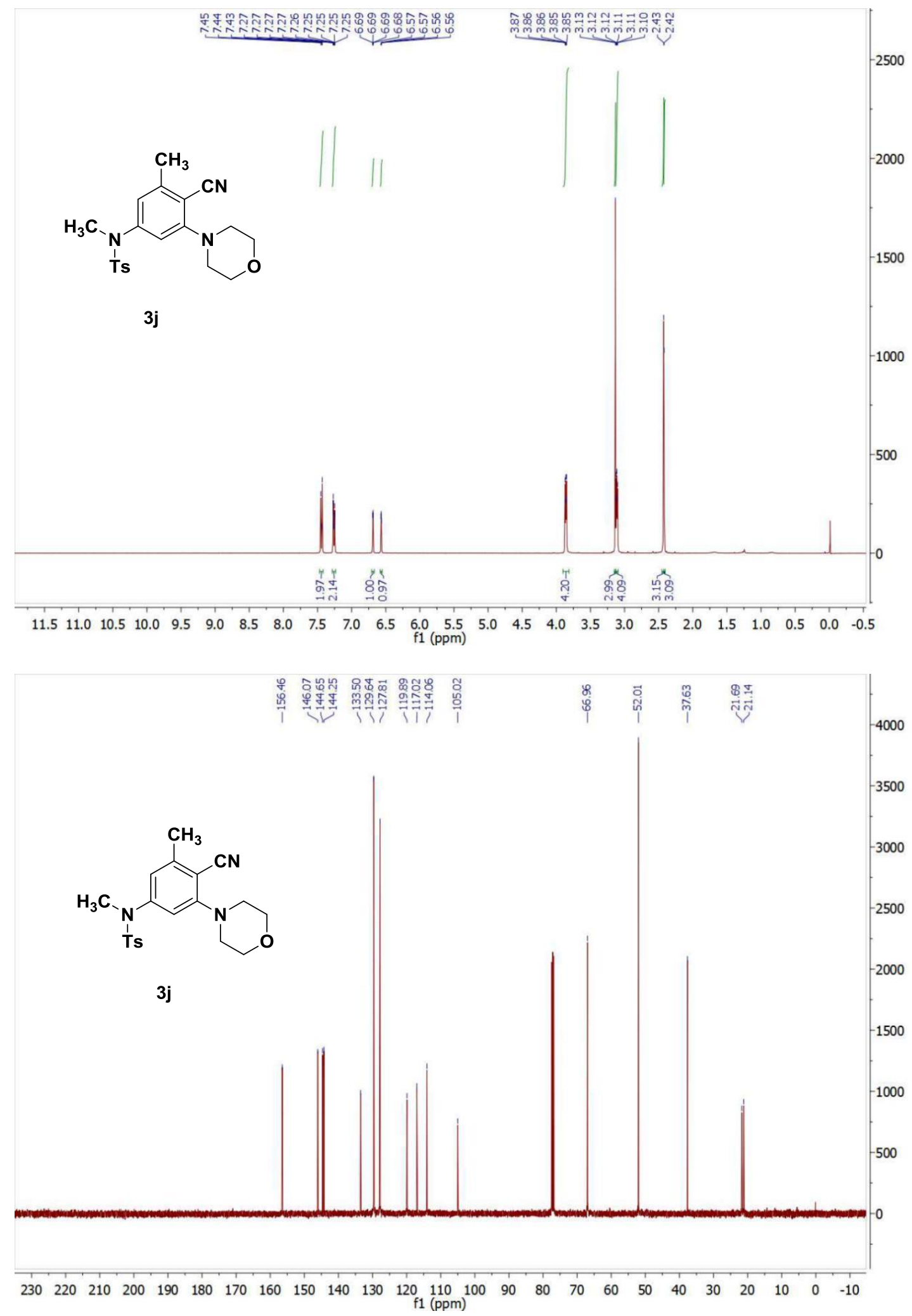




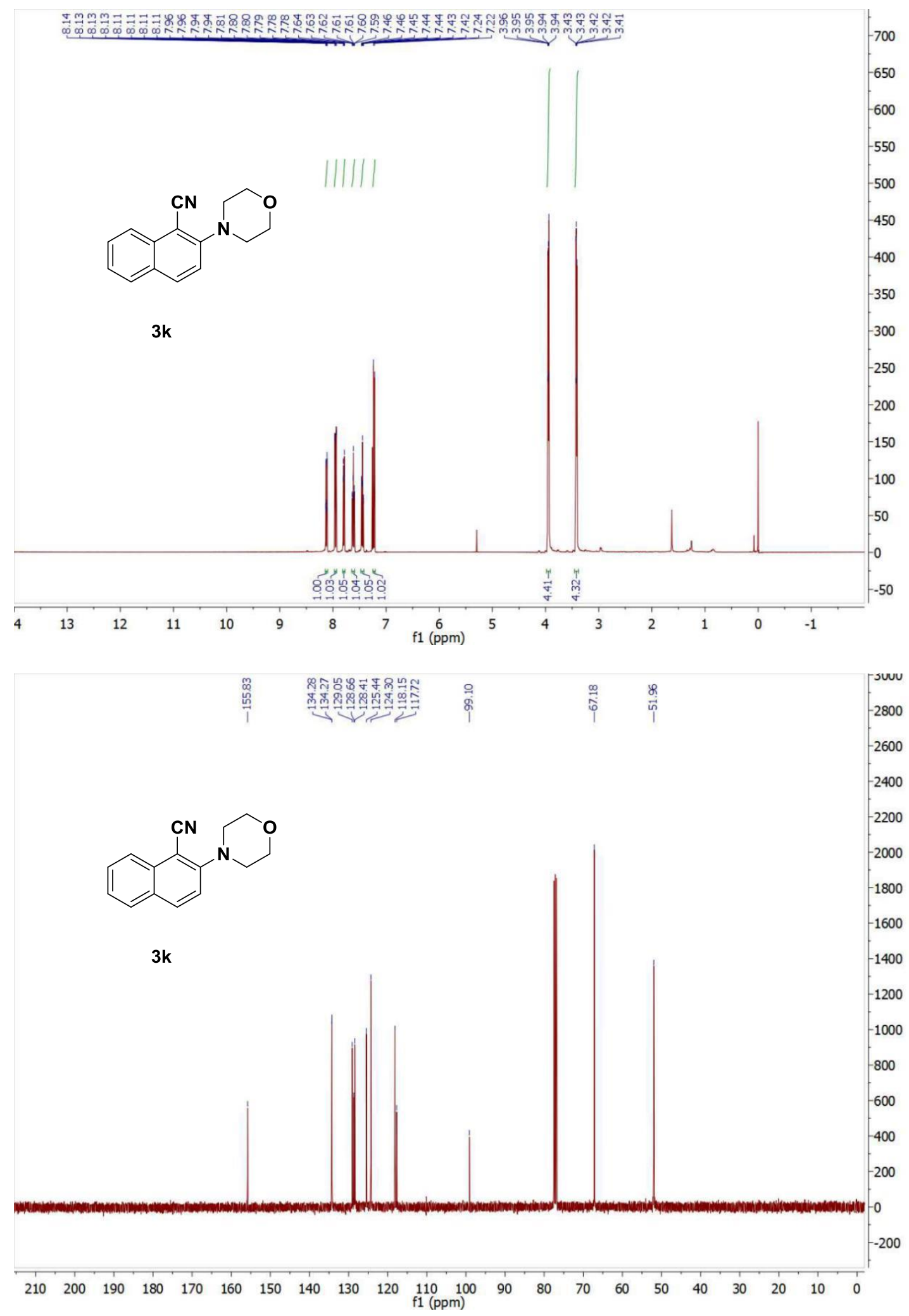



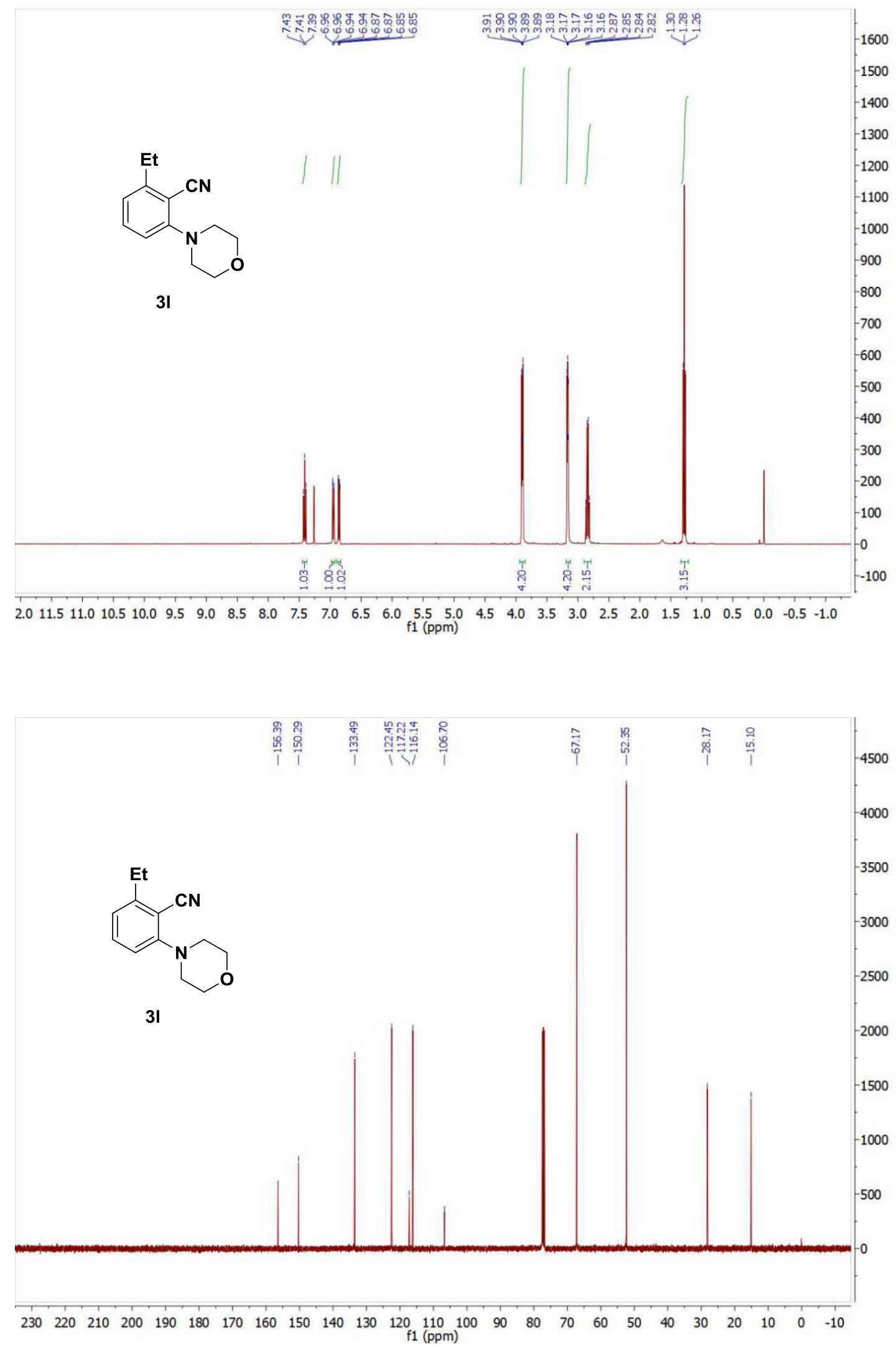

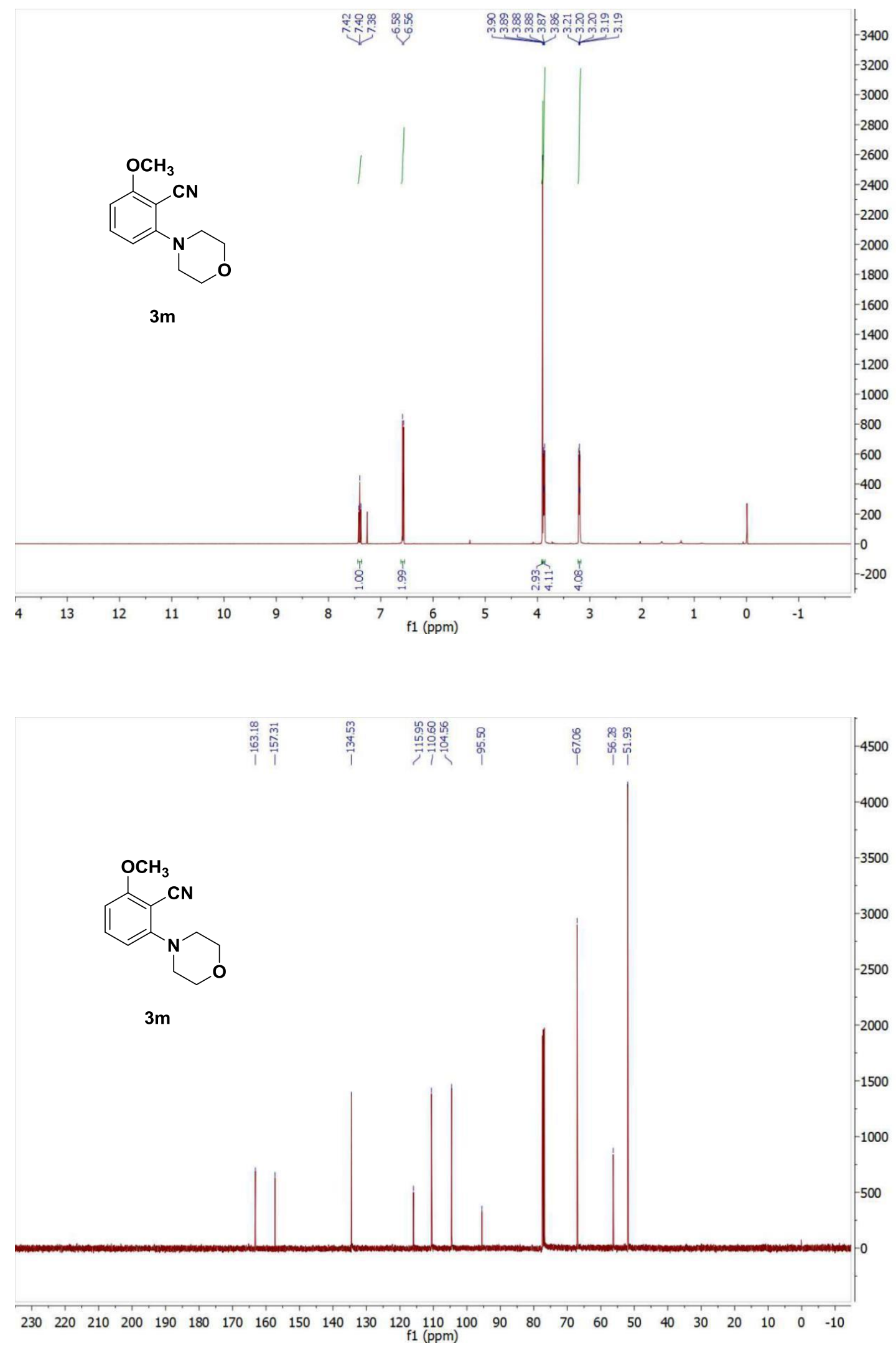

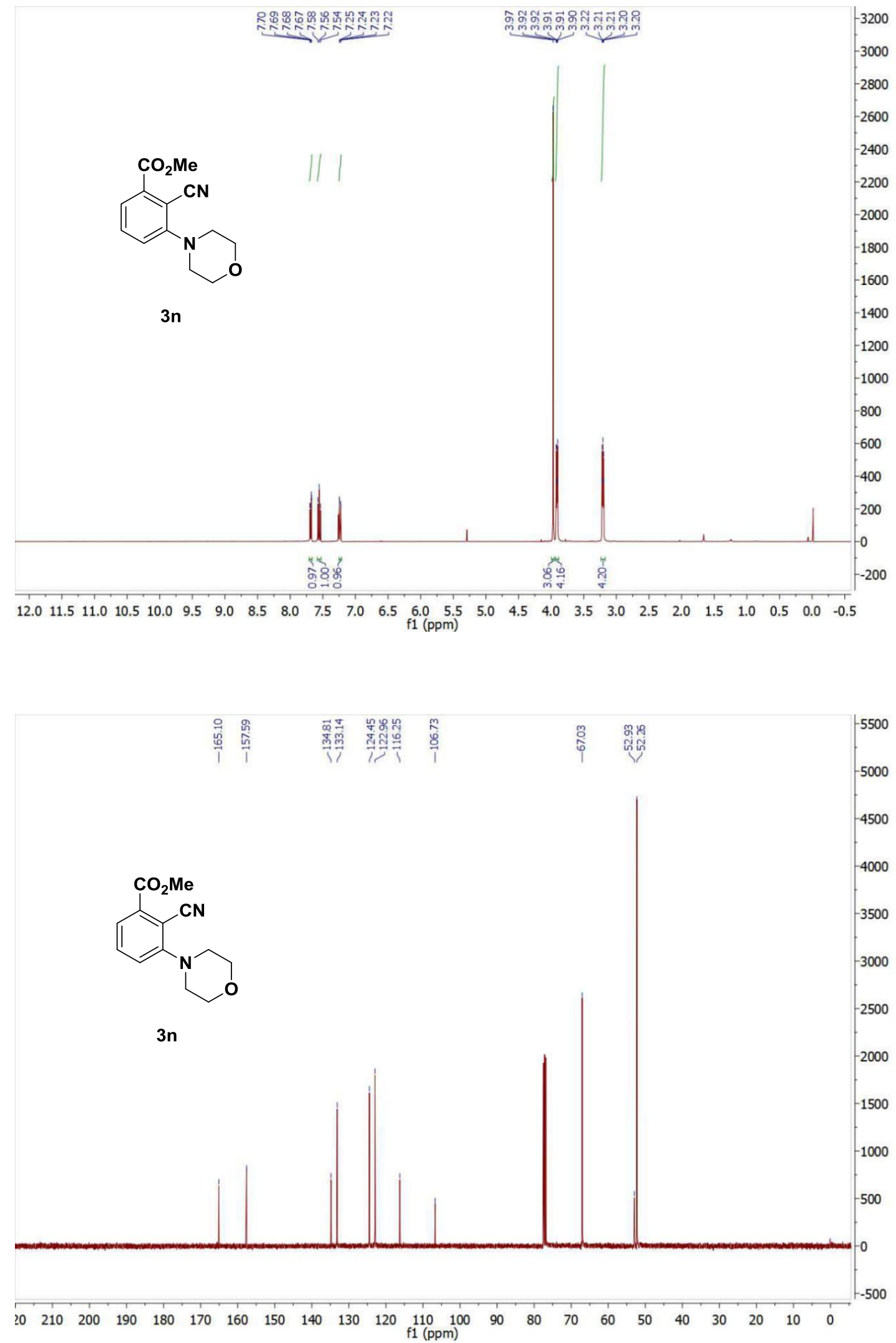


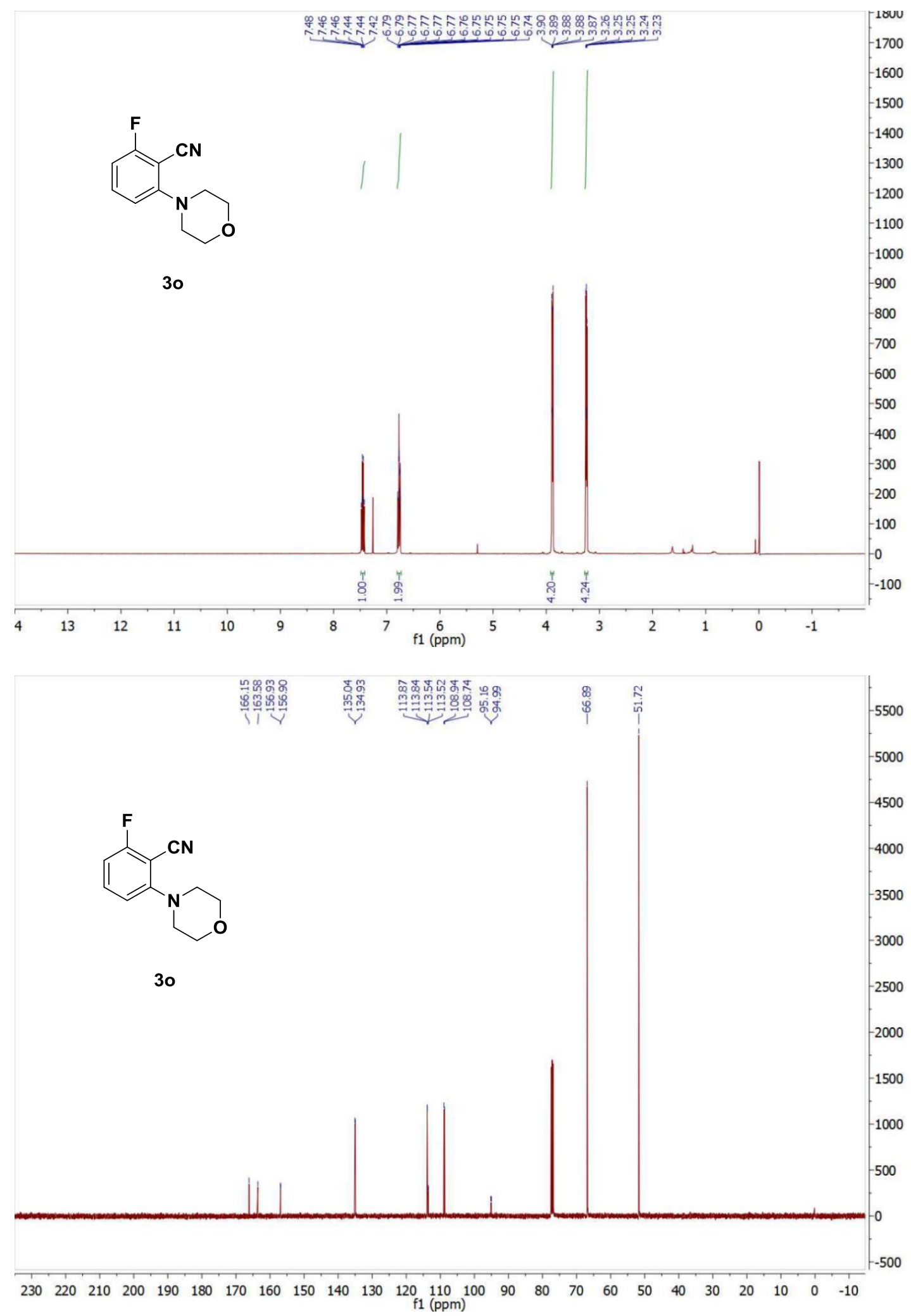



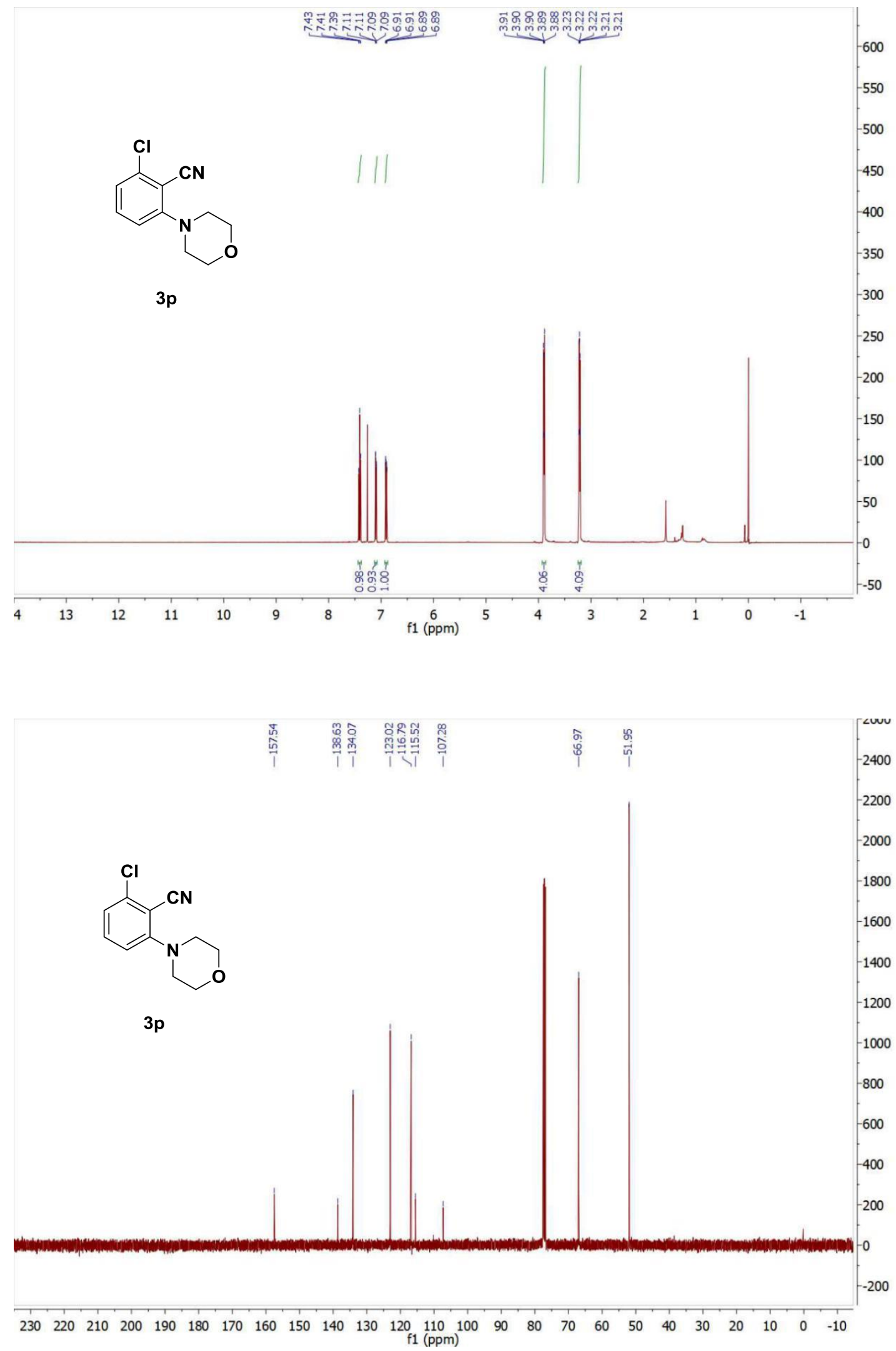

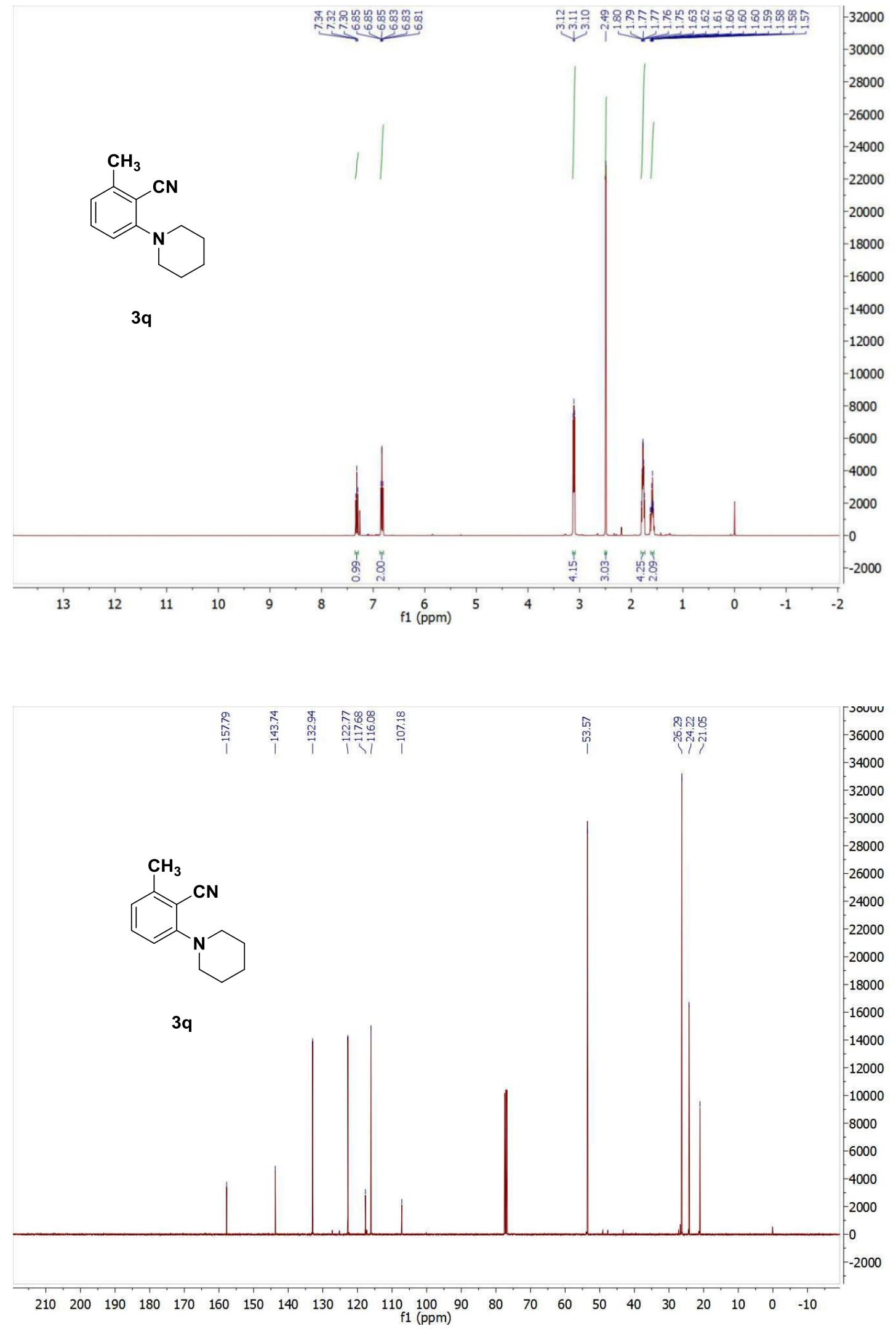


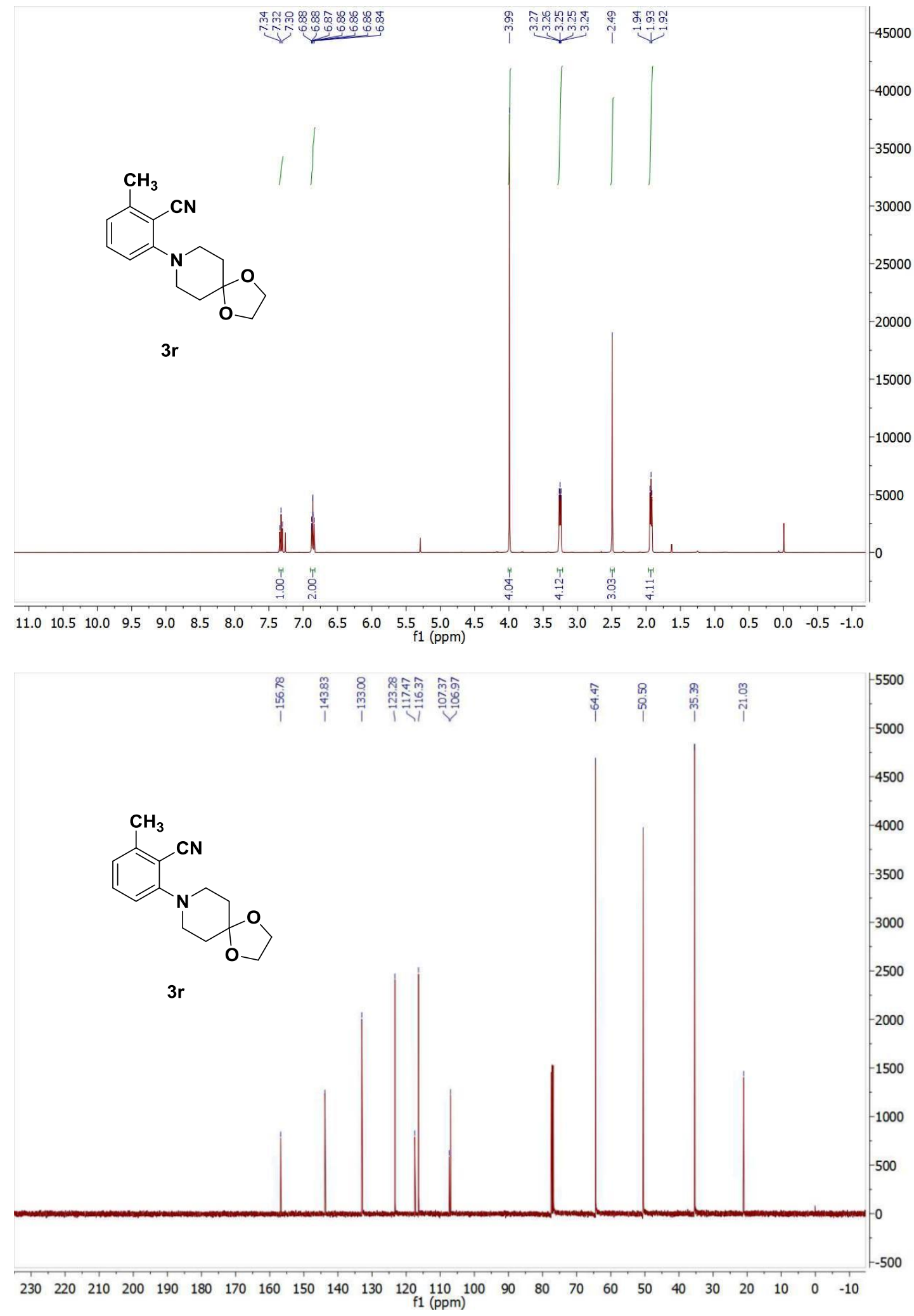




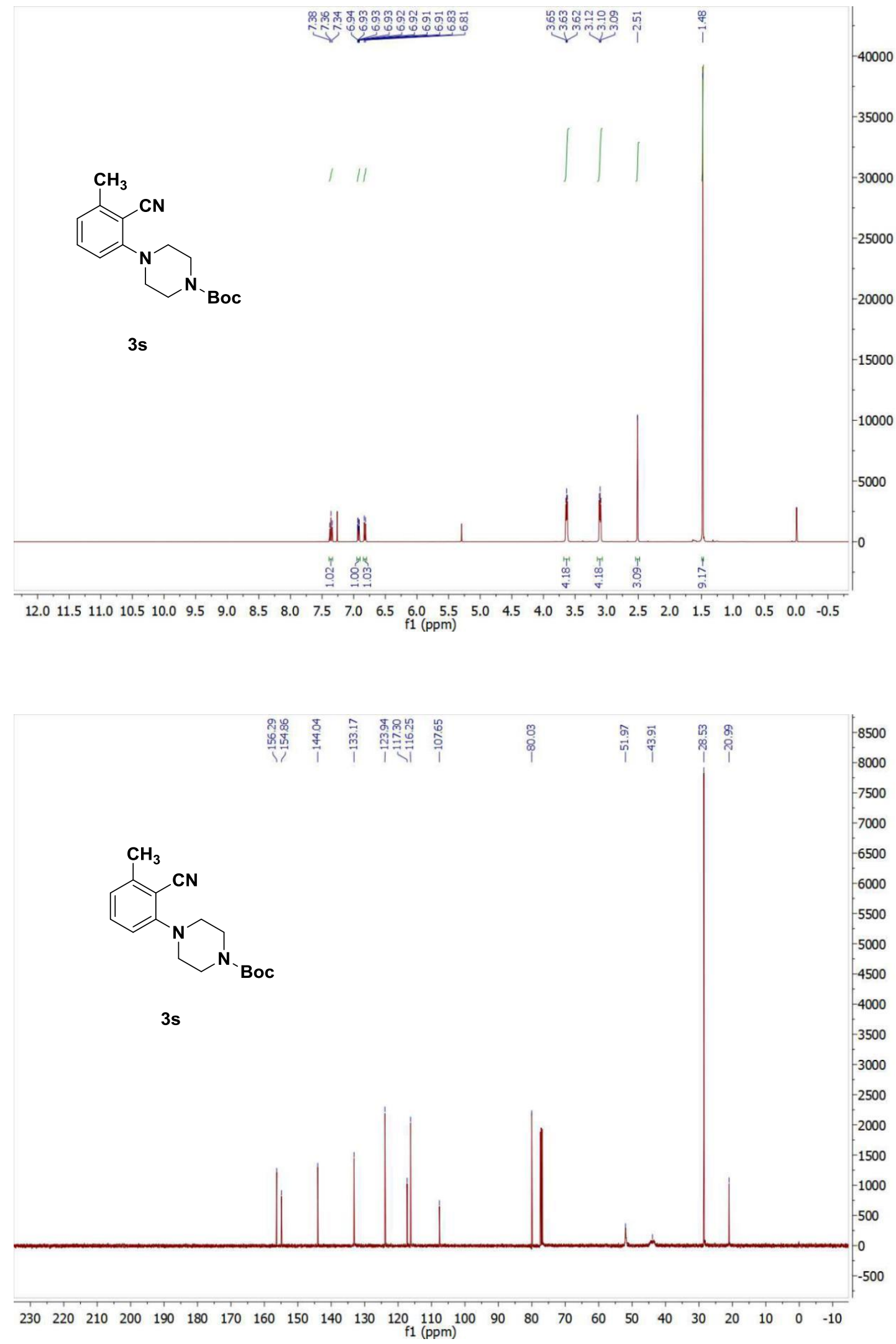



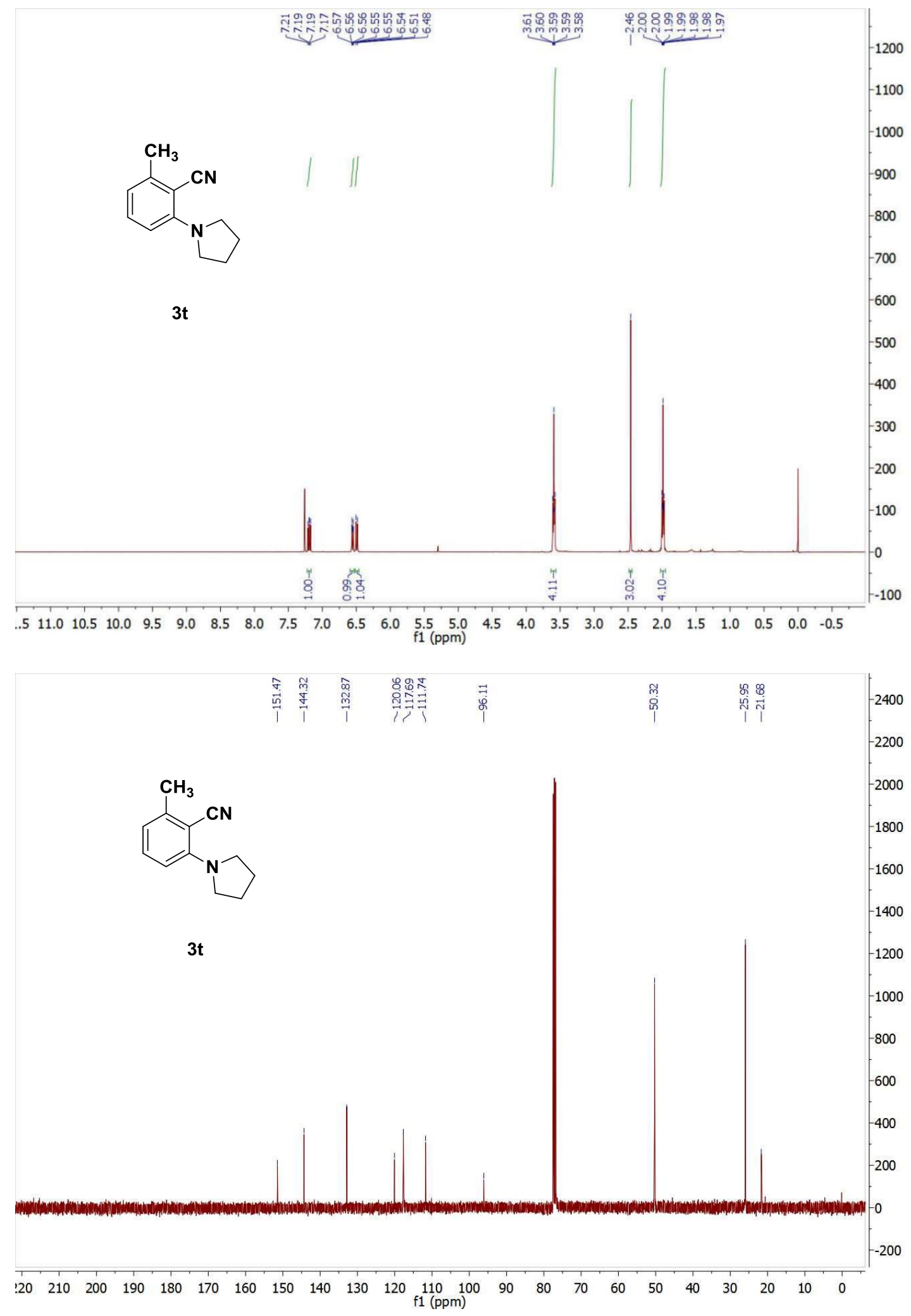

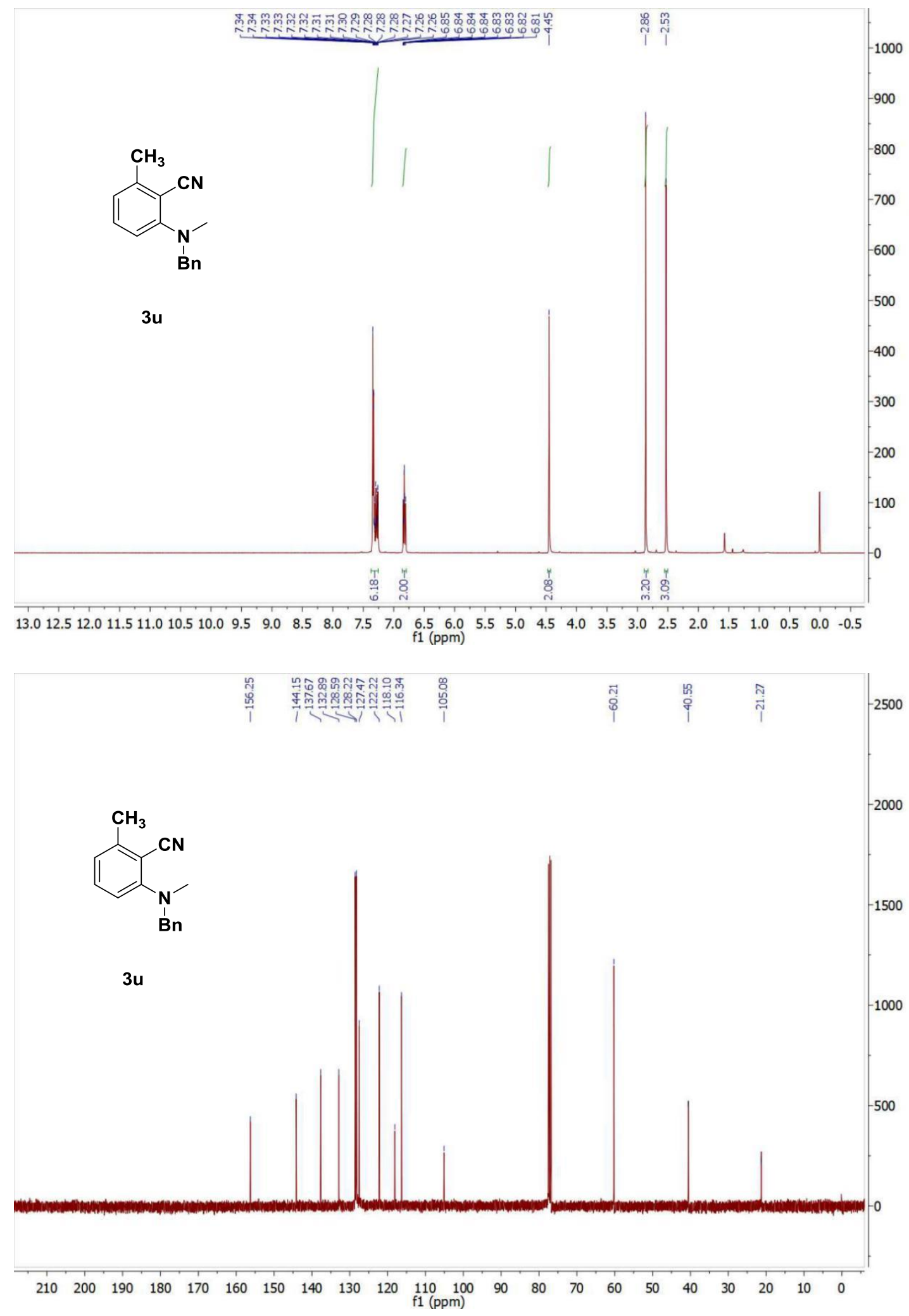

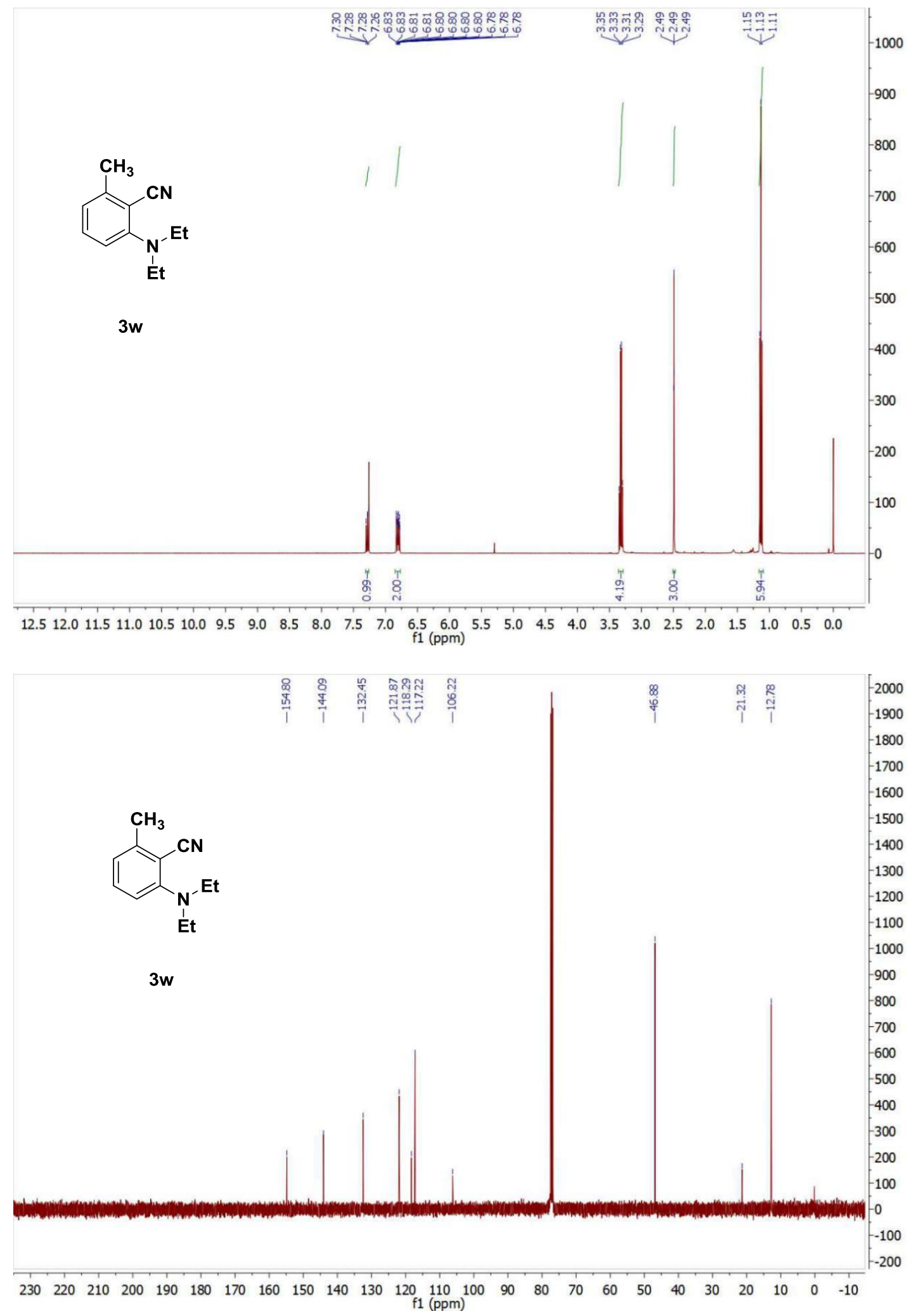

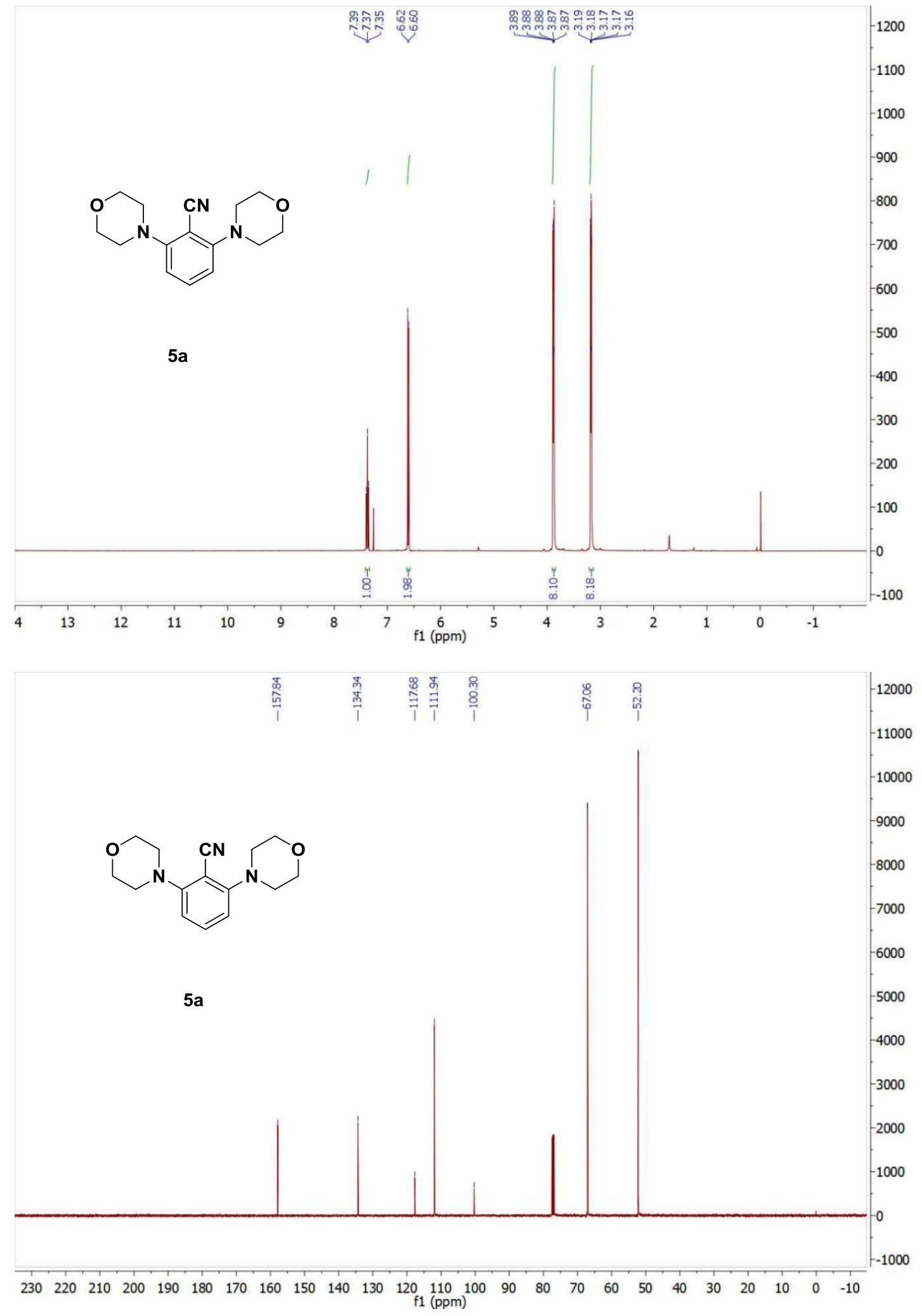


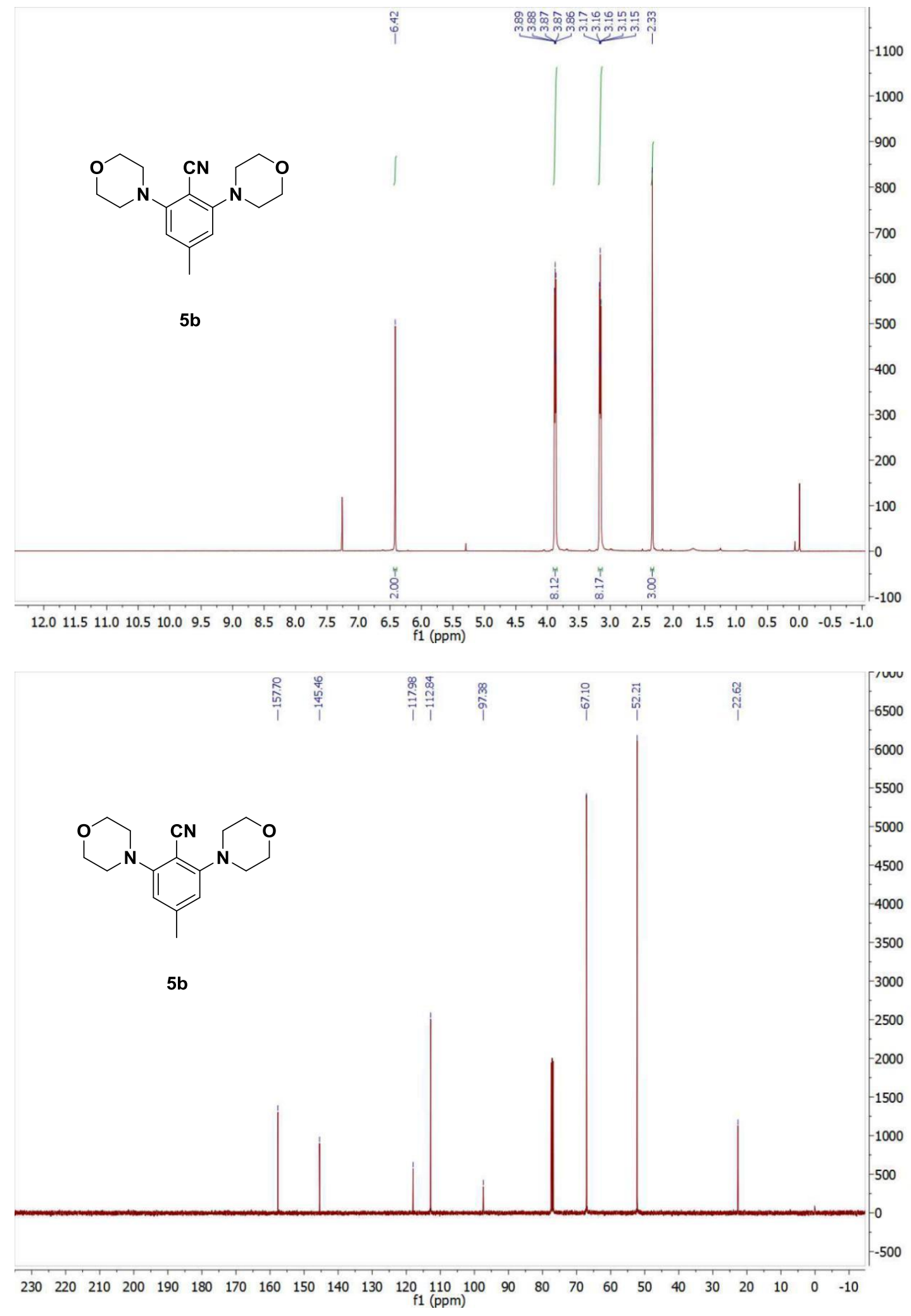




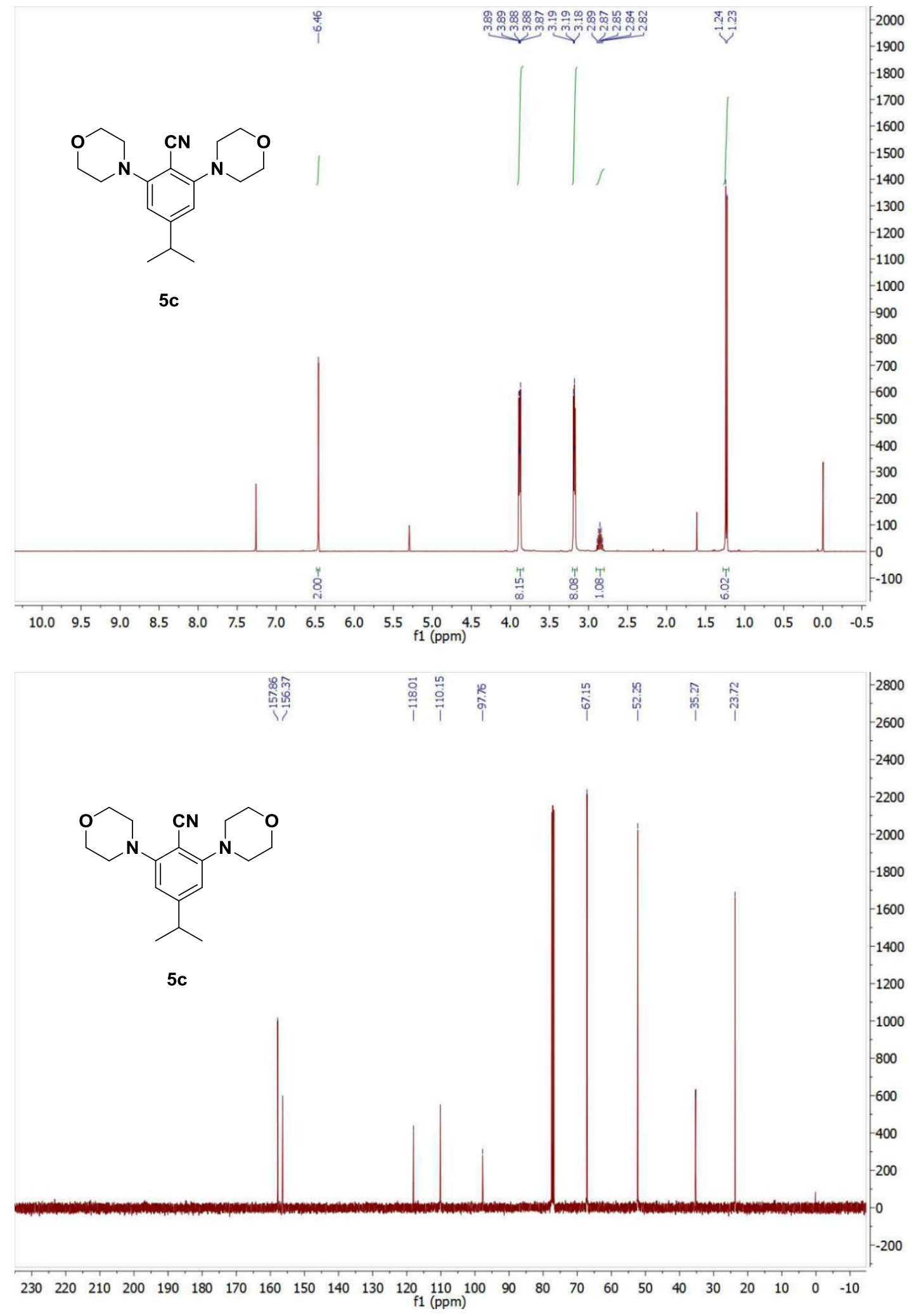



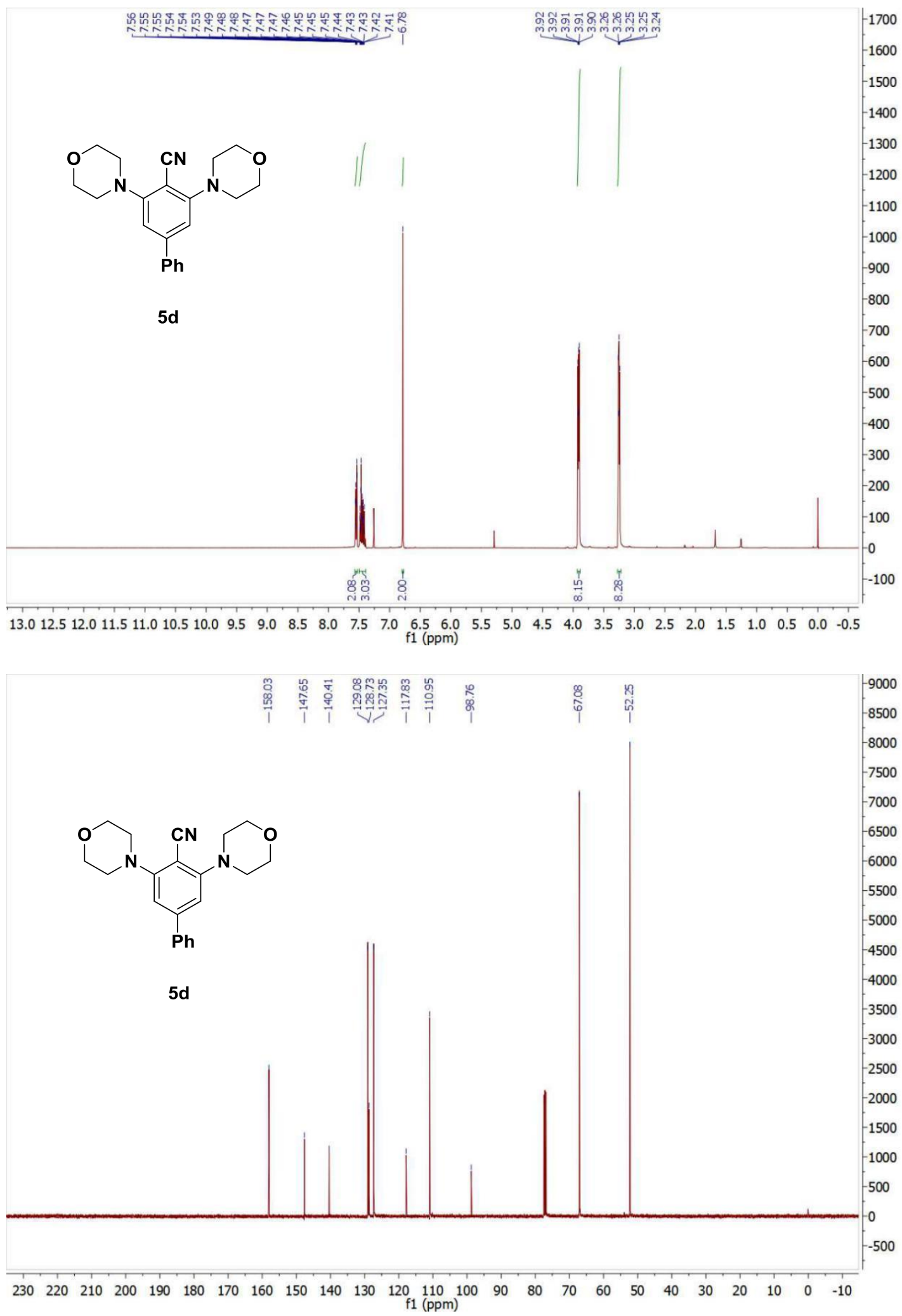


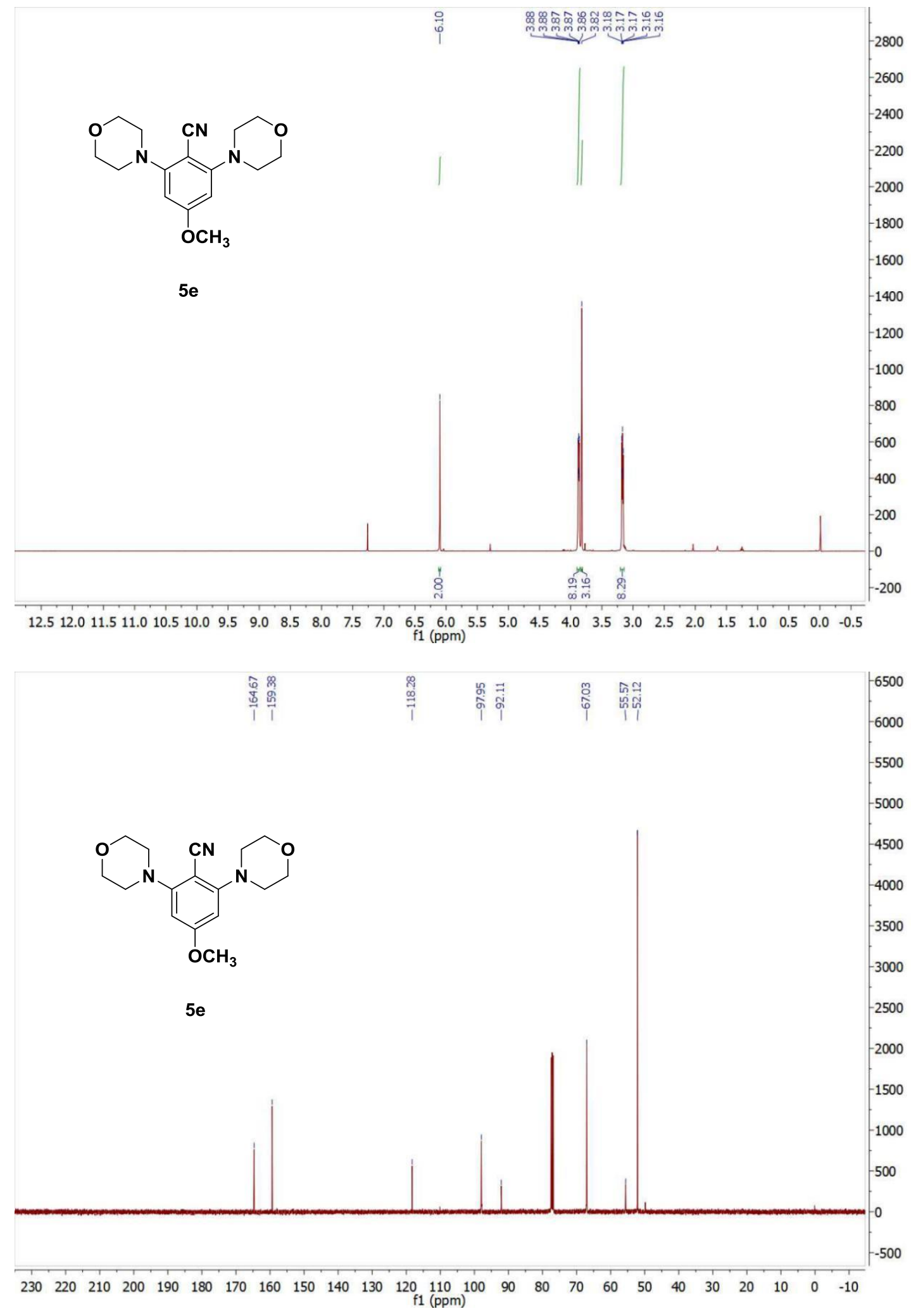



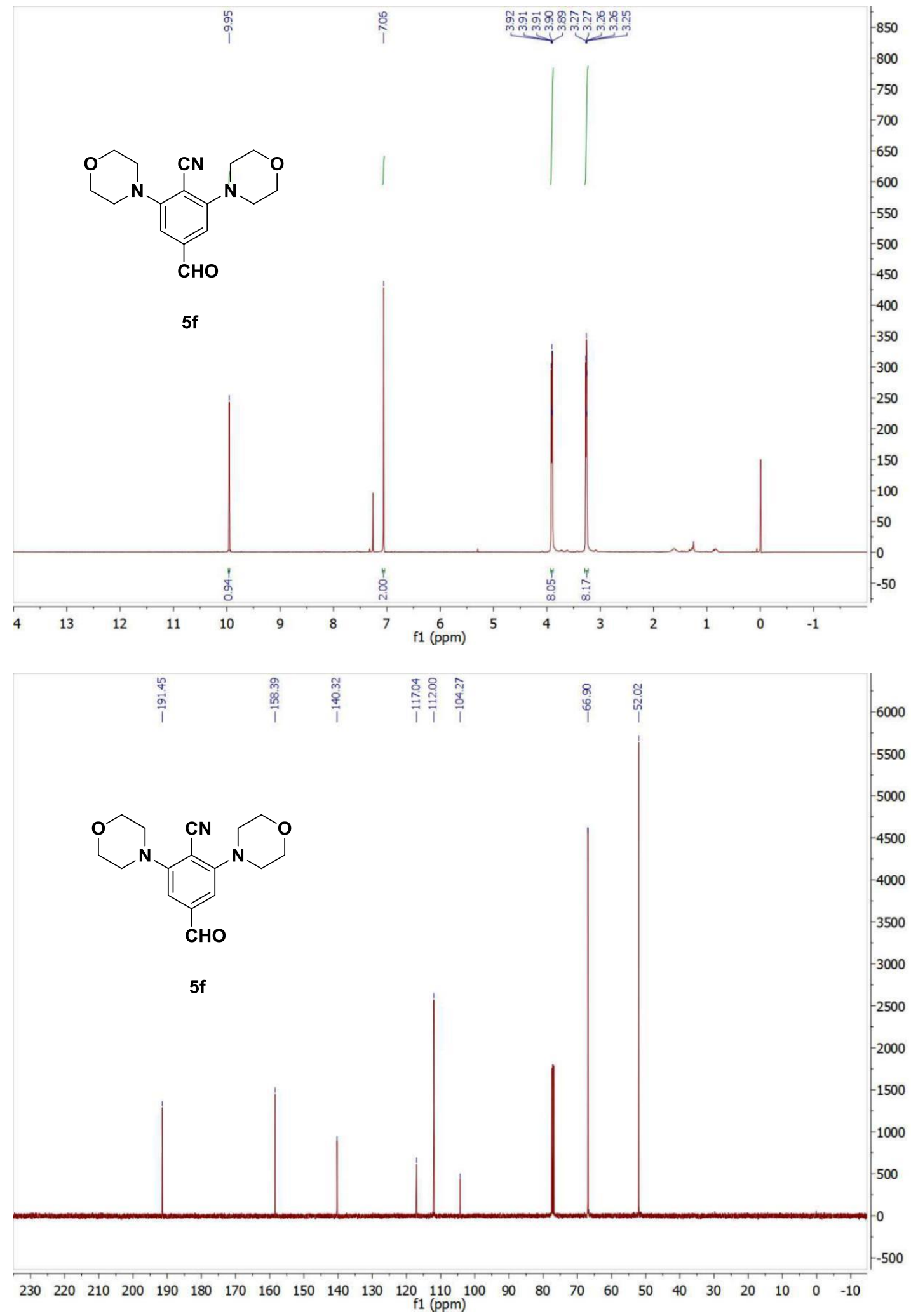

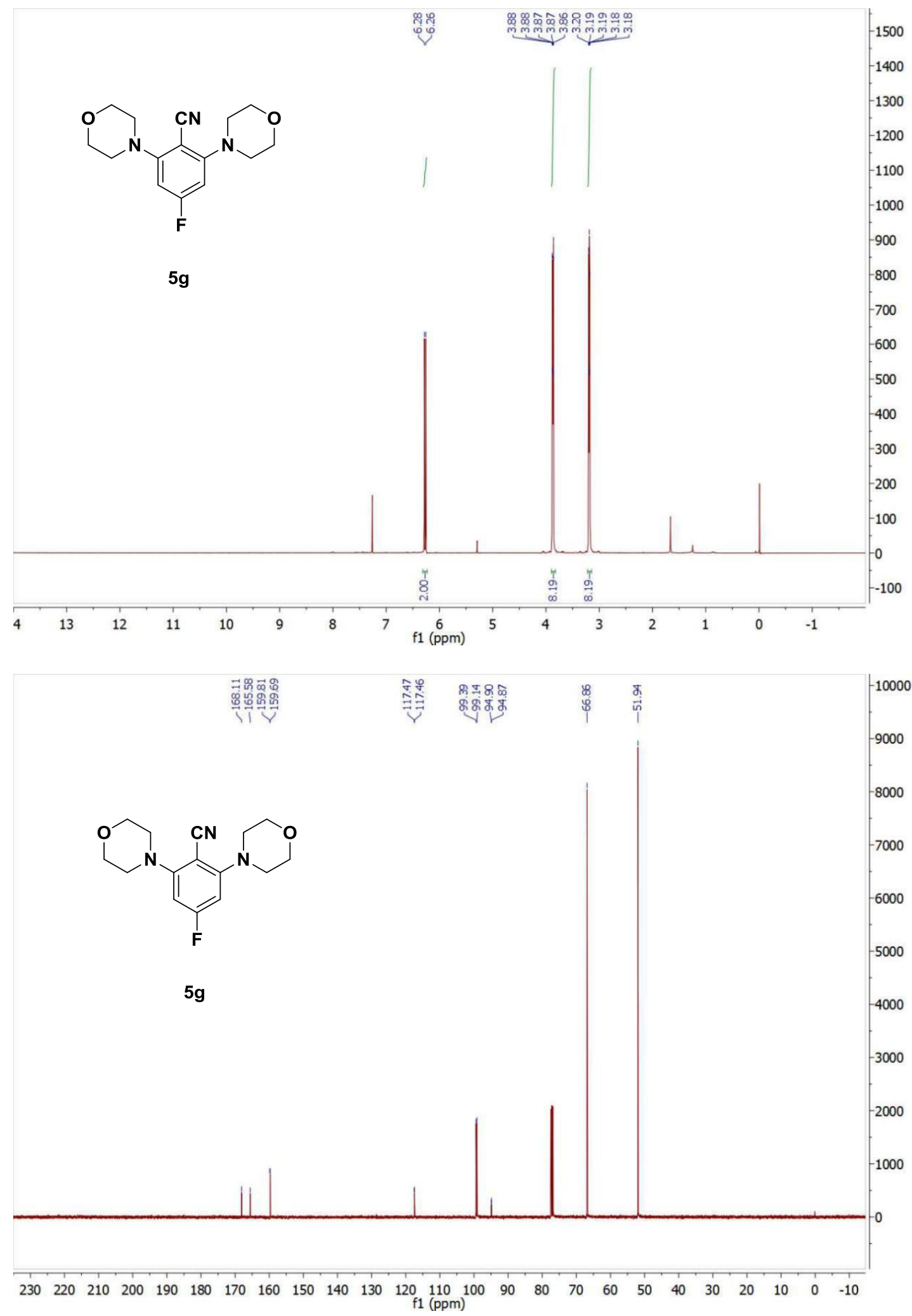

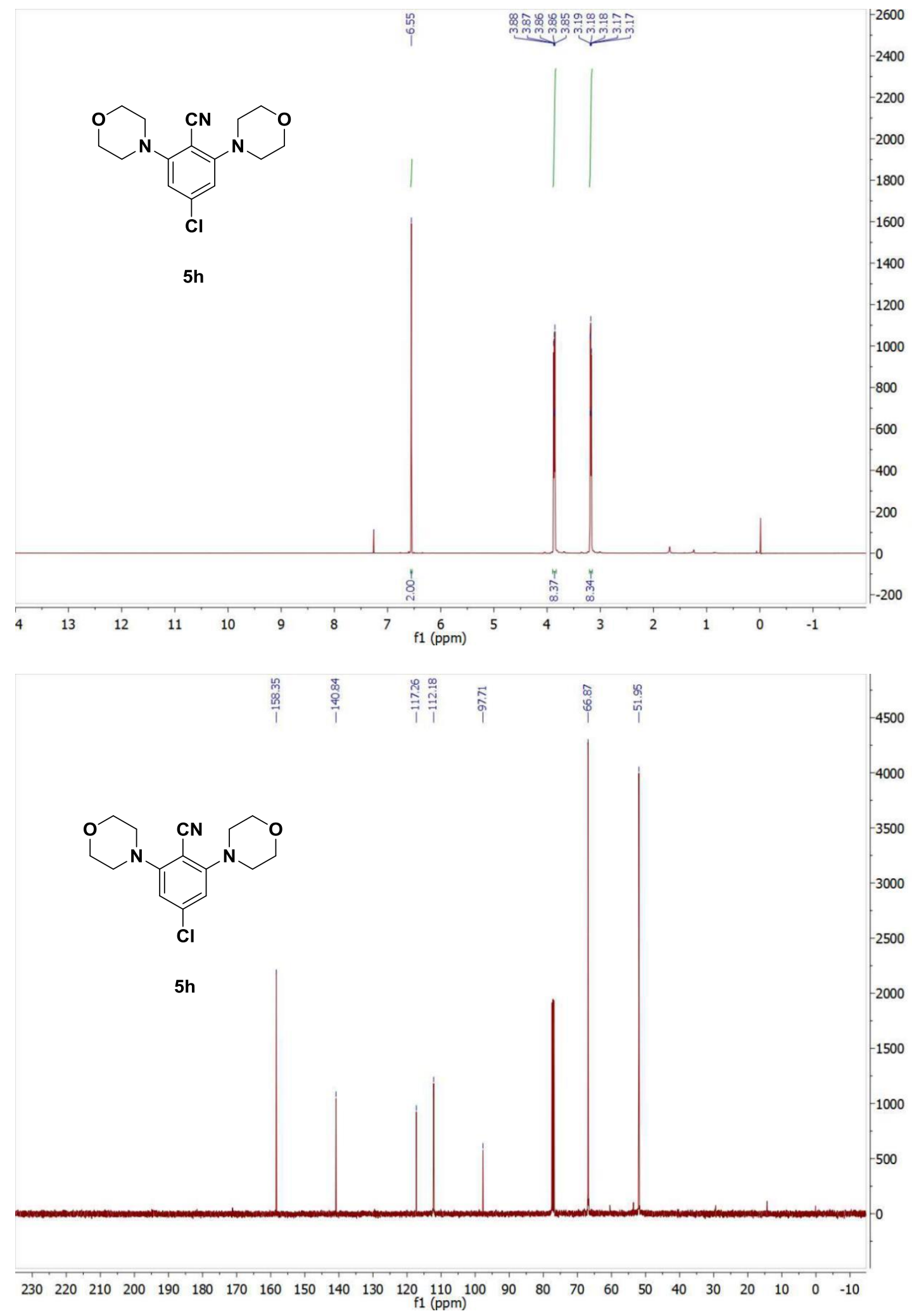


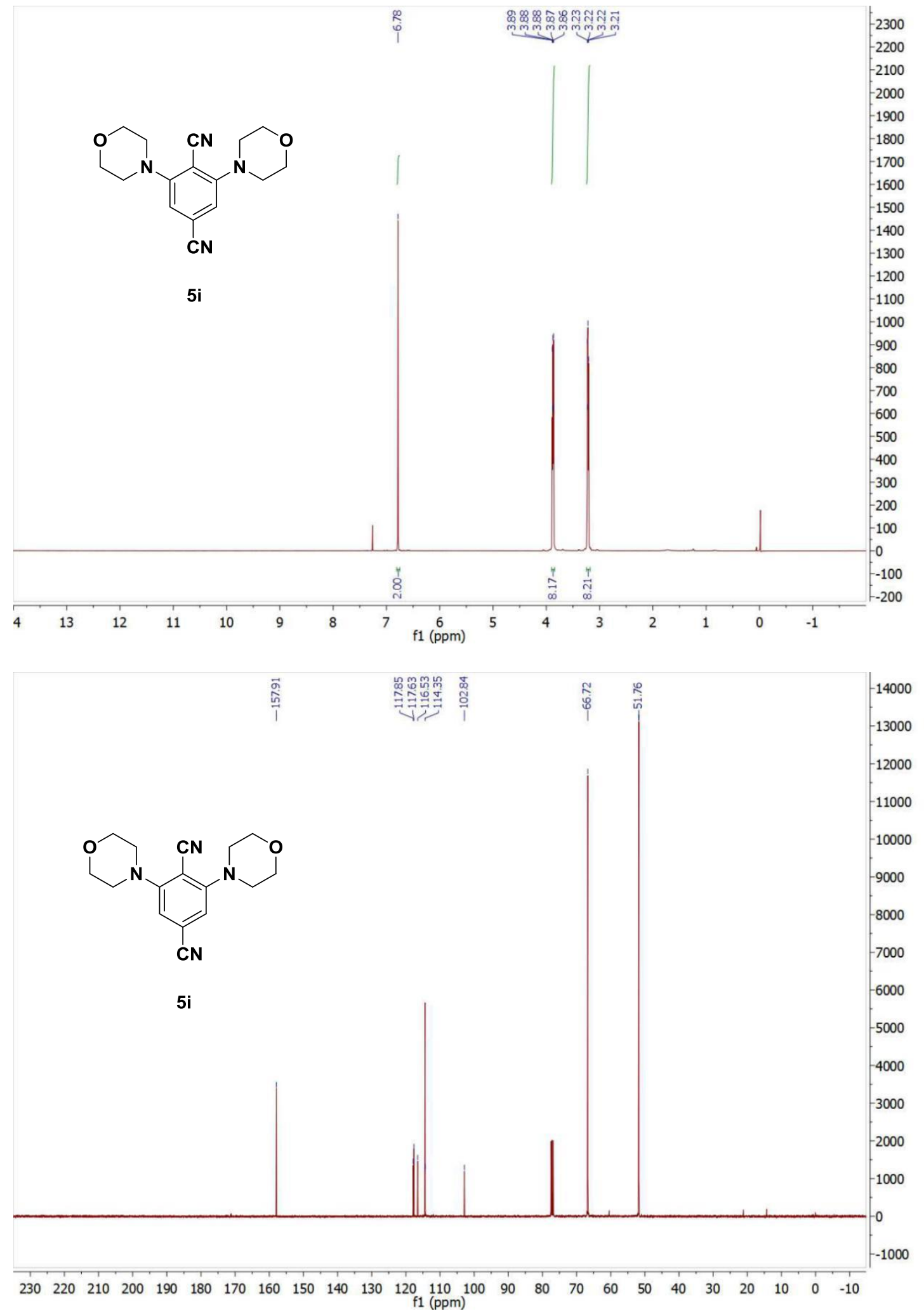




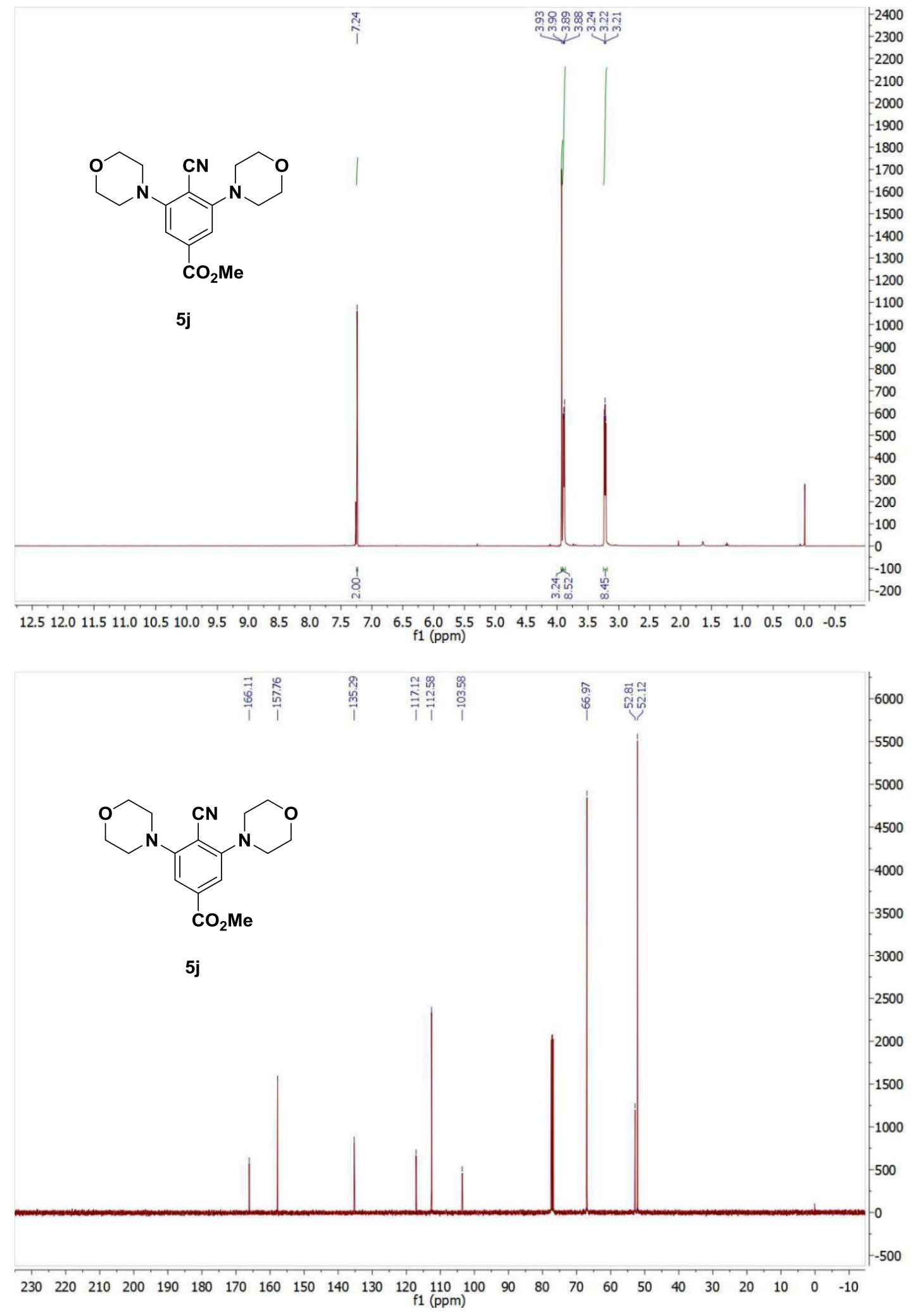




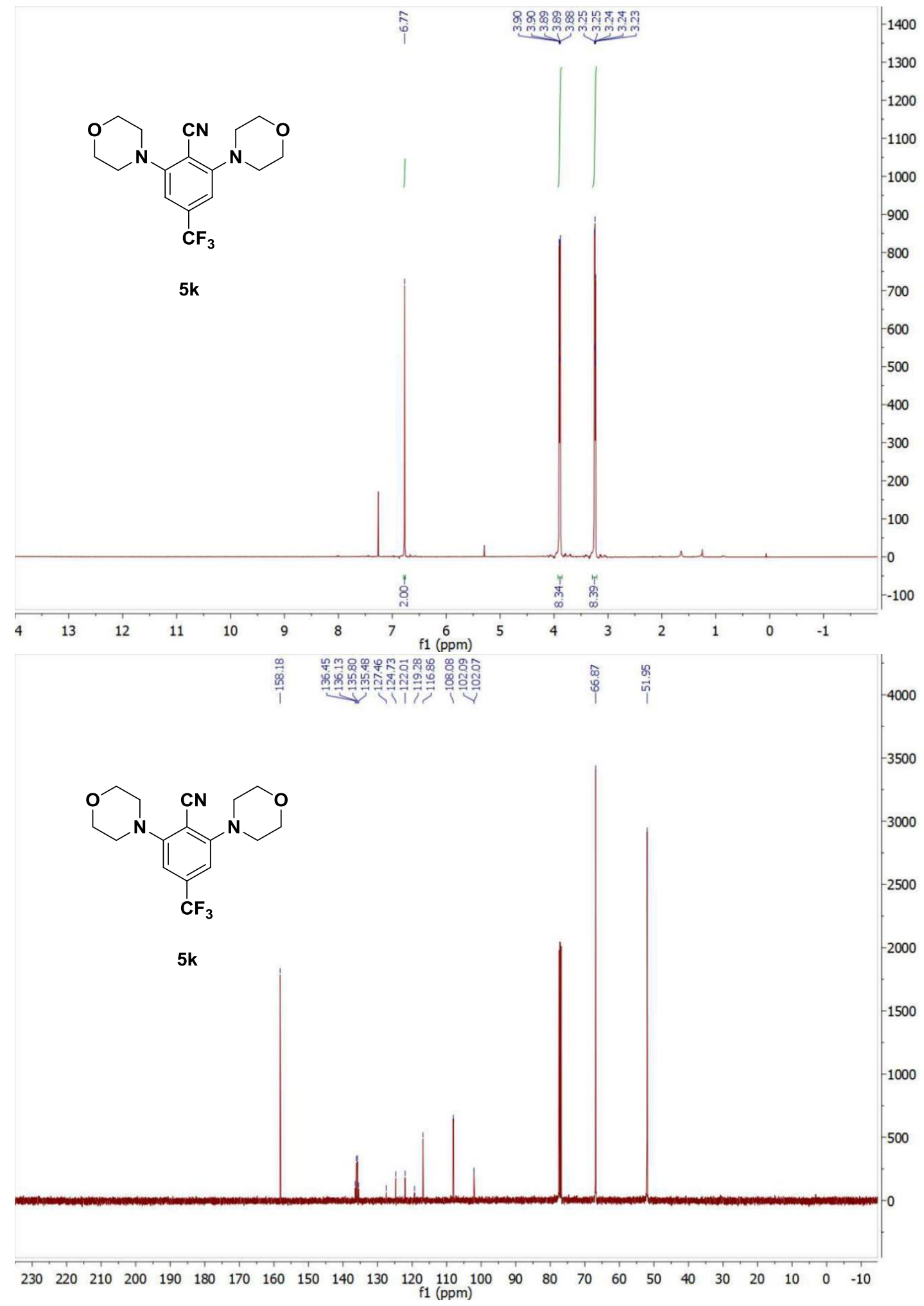



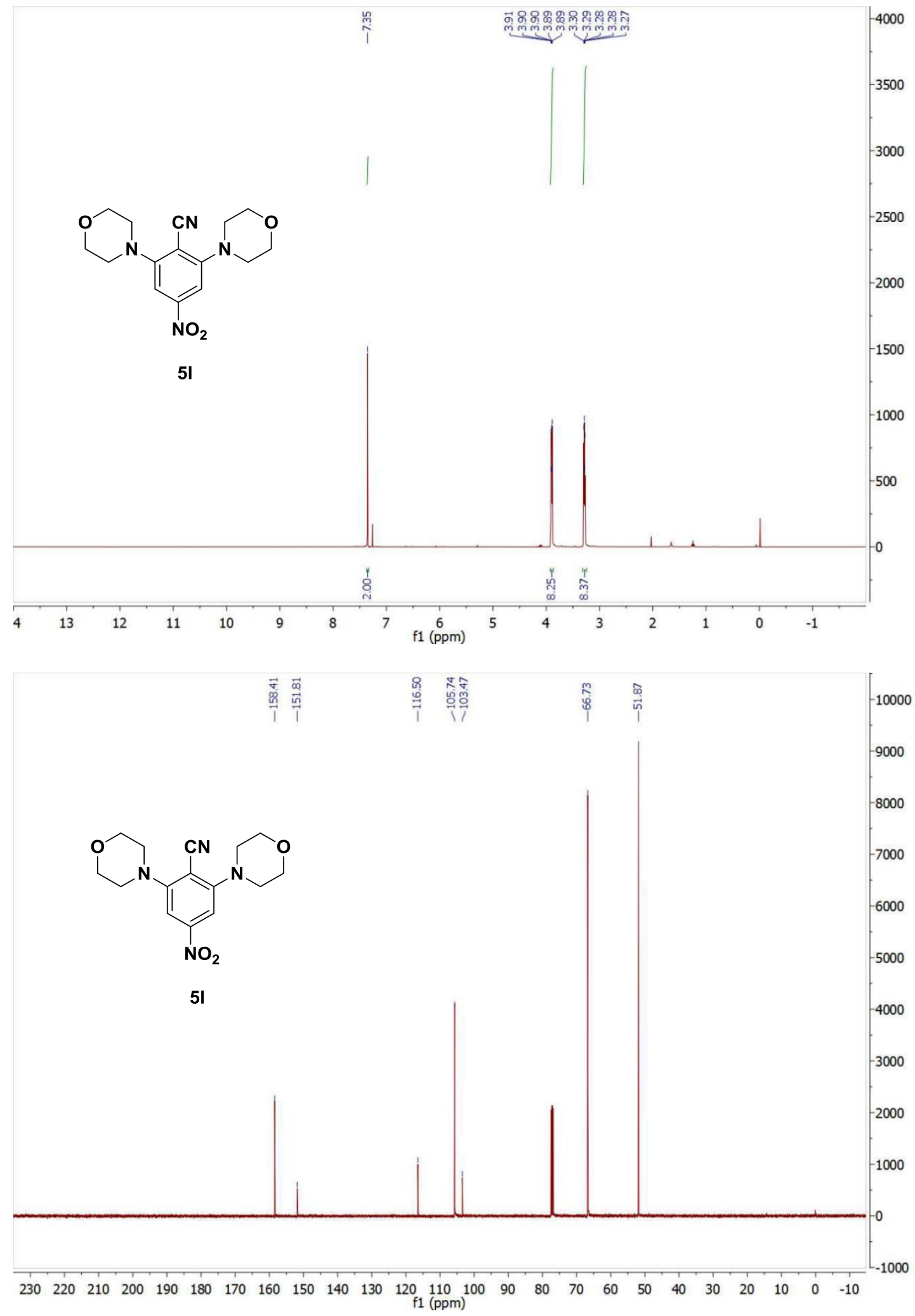
X-Ray Structures and Corresponding Data

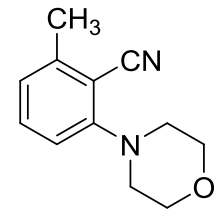

$3 a$

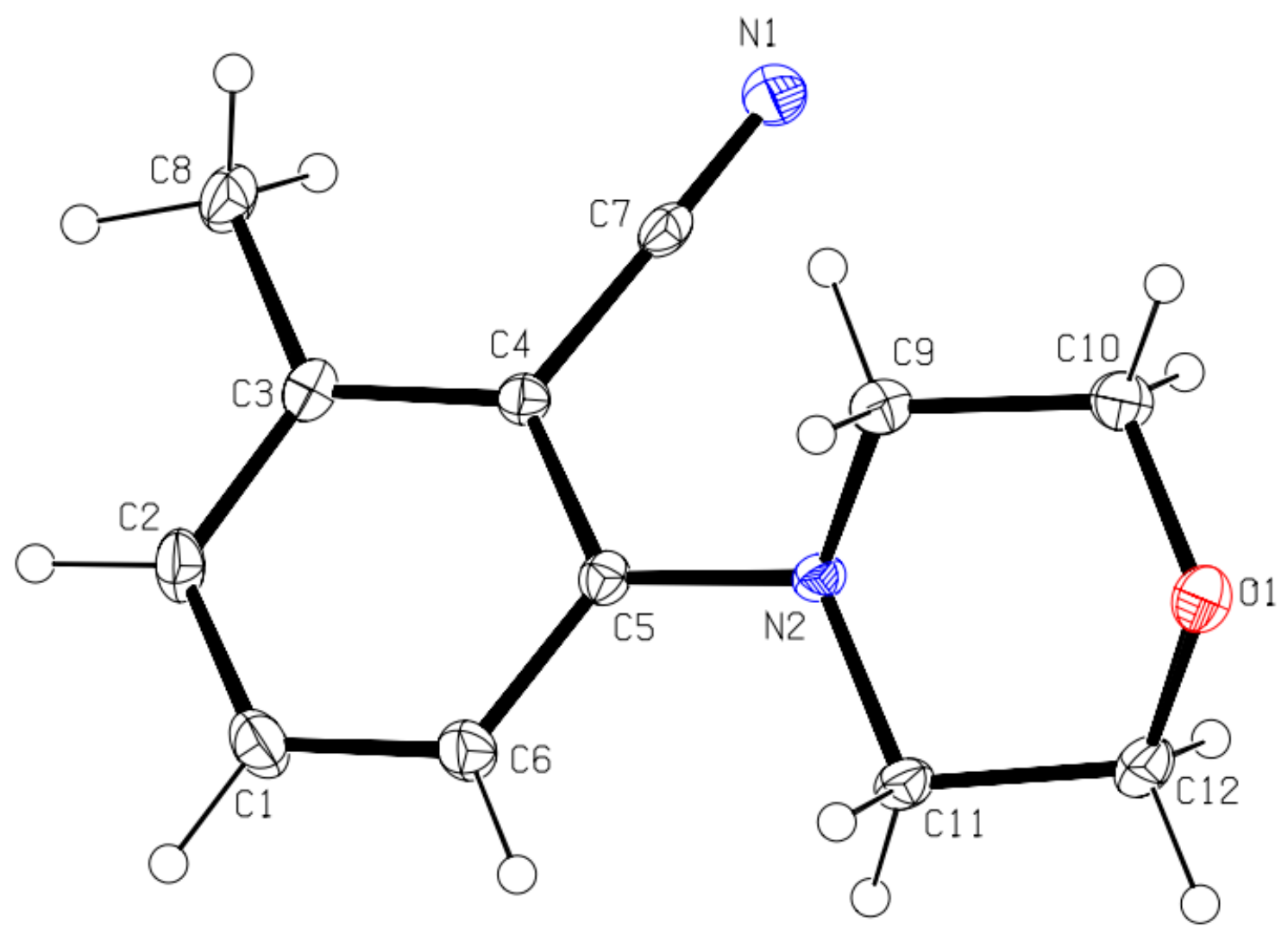


Table 1. Crystal data and structure refinement for d1680_a.

Identification code

Empirical formula

Formula weight

Temperature

Wavelength

Crystal system

Space group

Unit cell dimensions

Volume

Z

Density (calculated)

Absorption coefficient

$\mathrm{F}(000)$

Crystal size

Theta range for data collection

Index ranges

Reflections collected

Independent reflections

Completeness to theta $=25.242^{\circ}$

Absorption correction

Max. and min. transmission d1680_a

C12 H14 N2 O

202.25

147(2) K

$0.71073 \AA$

Monoclinic

Cc

$$
\begin{array}{ll}
\mathrm{a}=8.1402(13) \AA & \mathrm{a}=90^{\circ} . \\
\mathrm{b}=15.085(2) \AA & \mathrm{b}=104.569(5)^{\circ} . \\
\mathrm{c}=8.9672(14) \AA & \mathrm{g}=90^{\circ} .
\end{array}
$$

1065.7(3) $\AA^{3}$

4

$1.261 \mathrm{Mg} / \mathrm{m}^{3}$

$0.082 \mathrm{~mm}^{-1}$

432

$0.330 \times 0.230 \times 0.130 \mathrm{~mm}^{3}$

2.701 to $27.499^{\circ}$.

$-9<=\mathrm{h}<=10,-19<=\mathrm{k}<=19,-11<=\mathrm{l}<=11$

9447

$2362[\mathrm{R}($ int $)=0.0308]$

$100.0 \%$

Semi-empirical from equivalents

0.7456 and 0.6894 
Refinement method

Data / restraints / parameters

Goodness-of-fit on $\mathrm{F}^{2}$

Final R indices [I $>2 \operatorname{sigma}(\mathrm{I})]$

$\mathrm{R}$ indices (all data)

Extinction coefficient

Largest diff. peak and hole
Full-matrix least-squares on $\mathrm{F}^{2}$

$2362 / 2 / 137$

1.023

$\mathrm{R} 1=0.0369, \mathrm{wR} 2=0.0852$

$\mathrm{R} 1=0.0455, \mathrm{wR} 2=0.0897$

n/a

0.161 and -0.216 e. $\AA^{-3}$

Table 2. Atomic coordinates ( x 10 $)$ and equivalent isotropic displacement parameters $\left(\AA^{2} \times 10^{3}\right)$ for d1680_a. U(eq) is defined as one third of the trace of the orthogonalized $U^{i j}$ tensor.

\begin{tabular}{|c|c|c|c|c|}
\hline & $\mathrm{x}$ & $\mathrm{y}$ & $\mathrm{z}$ & $\mathrm{U}(\mathrm{eq})$ \\
\hline $\mathrm{O}(1)$ & $8536(2)$ & $4111(1)$ & $9069(2)$ & $28(1)$ \\
\hline $\mathrm{N}(1)$ & $7144(3)$ & $3158(2)$ & $3085(2)$ & $31(1)$ \\
\hline $\mathrm{N}(2)$ & $5786(2)$ & $3743(1)$ & $6459(2)$ & 19(1) \\
\hline $\mathrm{C}(1)$ & $1255(3)$ & $3947(2)$ & $4323(3)$ & $32(1)$ \\
\hline $\mathrm{C}(2)$ & $1355(3)$ & $3850(2)$ & $2814(3)$ & $28(1)$ \\
\hline $\mathrm{C}(3)$ & $2906(3)$ & $3706(2)$ & 2481(3) & $23(1)$ \\
\hline $\mathrm{C}(4)$ & $4366(3)$ & $3664(1)$ & $3723(3)$ & 19(1) \\
\hline$C(5)$ & $4287(3)$ & $3793(1)$ & $5272(2)$ & 19(1) \\
\hline$C(6)$ & $2689(3)$ & $3923(2)$ & $5536(3)$ & $26(1)$ \\
\hline $\mathrm{C}(7)$ & $5932(3)$ & $3395(2)$ & 3391(3) & 21(1) \\
\hline $\mathrm{C}(8)$ & $3029(3)$ & $3576(2)$ & $856(3)$ & $29(1)$ \\
\hline
\end{tabular}




\begin{tabular}{lrrrr}
$\mathrm{C}(9)$ & $7117(3)$ & $4394(2)$ & $6404(3)$ & $22(1)$ \\
$\mathrm{C}(10)$ & $8746(3)$ & $4129(2)$ & $7536(3)$ & $27(1)$ \\
$\mathrm{C}(11)$ & $5567(3)$ & $3694(2)$ & $8042(2)$ & $22(1)$ \\
$\mathrm{C}(12)$ & $7252(3)$ & $3475(2)$ & $9147(3)$ & $26(1)$ \\
\hline
\end{tabular}

Table 3. Bond lengths $[\AA]$ and angles $\left[^{\circ}\right]$ for d1680_a.

\begin{tabular}{ll}
\hline $\mathrm{O}(1)-\mathrm{C}(10)$ & $1.427(3)$ \\
$\mathrm{O}(1)-\mathrm{C}(12)$ & $1.433(3)$ \\
$\mathrm{N}(1)-\mathrm{C}(7)$ & $1.145(3)$ \\
$\mathrm{N}(2)-\mathrm{C}(5)$ & $1.405(3)$ \\
$\mathrm{N}(2)-\mathrm{C}(9)$ & $1.473(3)$ \\
$\mathrm{N}(2)-\mathrm{C}(11)$ & $1.476(3)$ \\
$\mathrm{C}(1)-\mathrm{C}(6)$ & $1.382(4)$ \\
$\mathrm{C}(1)-\mathrm{C}(2)$ & $1.384(4)$ \\
$\mathrm{C}(1)-\mathrm{H}(1 \mathrm{~A})$ & $1.421(3)$ \\
$\mathrm{C}(2)-\mathrm{C}(3)$ & 0.9500 \\
$\mathrm{C}(2)-\mathrm{H}(2 \mathrm{~A})$ & $1.386(3)$ \\
$\mathrm{C}(3)-\mathrm{C}(4)$ & 0.9500 \\
$\mathrm{C}(3)-\mathrm{C}(8)$ & $1.411(3)$ \\
$\mathrm{C}(4)-\mathrm{C}(5)$ & $1.498(3)$ \\
& \\
\hline
\end{tabular}




\begin{tabular}{|c|c|}
\hline $\mathrm{C}(8)-\mathrm{H}(8 \mathrm{~A})$ & 0.9800 \\
\hline $\mathrm{C}(8)-\mathrm{H}(8 \mathrm{~B})$ & 0.9800 \\
\hline $\mathrm{C}(8)-\mathrm{H}(8 \mathrm{C})$ & 0.9800 \\
\hline $\mathrm{C}(9)-\mathrm{C}(10)$ & $1.508(3)$ \\
\hline $\mathrm{C}(9)-\mathrm{H}(9 \mathrm{~A})$ & 0.9900 \\
\hline $\mathrm{C}(9)-\mathrm{H}(9 \mathrm{~B})$ & 0.9900 \\
\hline $\mathrm{C}(10)-\mathrm{H}(10 \mathrm{~A})$ & 0.9900 \\
\hline $\mathrm{C}(10)-\mathrm{H}(10 \mathrm{~B})$ & 0.9900 \\
\hline$C(11)-C(12)$ & $1.512(3)$ \\
\hline $\mathrm{C}(11)-\mathrm{H}(11 \mathrm{~A})$ & 0.9900 \\
\hline $\mathrm{C}(11)-\mathrm{H}(11 \mathrm{~B})$ & 0.9900 \\
\hline $\mathrm{C}(12)-\mathrm{H}(12 \mathrm{~A})$ & 0.9900 \\
\hline $\mathrm{C}(12)-\mathrm{H}(12 \mathrm{~B})$ & 0.9900 \\
\hline $\mathrm{C}(10)-\mathrm{O}(1)-\mathrm{C}(12)$ & $108.96(17)$ \\
\hline $\mathrm{C}(5)-\mathrm{N}(2)-\mathrm{C}(9)$ & $116.40(17)$ \\
\hline $\mathrm{C}(5)-\mathrm{N}(2)-\mathrm{C}(11)$ & $116.06(18)$ \\
\hline $\mathrm{C}(9)-\mathrm{N}(2)-\mathrm{C}(11)$ & $109.47(17)$ \\
\hline$C(6)-C(1)-C(2)$ & $121.4(2)$ \\
\hline $\mathrm{C}(6)-\mathrm{C}(1)-\mathrm{H}(1 \mathrm{~A})$ & 119.3 \\
\hline $\mathrm{C}(2)-\mathrm{C}(1)-\mathrm{H}(1 \mathrm{~A})$ & 119.3 \\
\hline $\mathrm{C}(1)-\mathrm{C}(2)-\mathrm{C}(3)$ & $120.5(2)$ \\
\hline $\mathrm{C}(1)-\mathrm{C}(2)-\mathrm{H}(2 \mathrm{~A})$ & 119.7 \\
\hline
\end{tabular}




\begin{tabular}{|c|c|}
\hline $\mathrm{C}(3)-\mathrm{C}(2)-\mathrm{H}(2 \mathrm{~A})$ & 119.7 \\
\hline$C(2)-C(3)-C(4)$ & $118.0(2)$ \\
\hline$C(2)-C(3)-C(8)$ & $121.1(2)$ \\
\hline $\mathrm{C}(4)-\mathrm{C}(3)-\mathrm{C}(8)$ & $120.8(2)$ \\
\hline $\mathrm{C}(3)-\mathrm{C}(4)-\mathrm{C}(5)$ & $121.9(2)$ \\
\hline$C(3)-C(4)-C(7)$ & $117.7(2)$ \\
\hline$C(5)-C(4)-C(7)$ & $120.1(2)$ \\
\hline $\mathrm{C}(6)-\mathrm{C}(5)-\mathrm{N}(2)$ & $123.28(19)$ \\
\hline$C(6)-C(5)-C(4)$ & 117.38(19) \\
\hline $\mathrm{N}(2)-\mathrm{C}(5)-\mathrm{C}(4)$ & 119.28(19) \\
\hline$C(1)-C(6)-C(5)$ & $120.6(2)$ \\
\hline $\mathrm{C}(1)-\mathrm{C}(6)-\mathrm{H}(6 \mathrm{~A})$ & 119.7 \\
\hline$C(5)-C(6)-H(6 A)$ & 119.7 \\
\hline $\mathrm{N}(1)-\mathrm{C}(7)-\mathrm{C}(4)$ & $177.3(2)$ \\
\hline $\mathrm{C}(3)-\mathrm{C}(8)-\mathrm{H}(8 \mathrm{~A})$ & 109.5 \\
\hline $\mathrm{C}(3)-\mathrm{C}(8)-\mathrm{H}(8 \mathrm{~B})$ & 109.5 \\
\hline $\mathrm{H}(8 \mathrm{~A})-\mathrm{C}(8)-\mathrm{H}(8 \mathrm{~B})$ & 109.5 \\
\hline $\mathrm{C}(3)-\mathrm{C}(8)-\mathrm{H}(8 \mathrm{C})$ & 109.5 \\
\hline $\mathrm{H}(8 \mathrm{~A})-\mathrm{C}(8)-\mathrm{H}(8 \mathrm{C})$ & 109.5 \\
\hline $\mathrm{H}(8 \mathrm{~B})-\mathrm{C}(8)-\mathrm{H}(8 \mathrm{C})$ & 109.5 \\
\hline $\mathrm{N}(2)-\mathrm{C}(9)-\mathrm{C}(10)$ & 109.23(18) \\
\hline $\mathrm{N}(2)-\mathrm{C}(9)-\mathrm{H}(9 \mathrm{~A})$ & 109.8 \\
\hline $\mathrm{C}(10)-\mathrm{C}(9)-\mathrm{H}(9 \mathrm{~A})$ & 109.8 \\
\hline
\end{tabular}




\begin{tabular}{|c|c|}
\hline $\mathrm{N}(2)-\mathrm{C}(9)-\mathrm{H}(9 \mathrm{~B})$ & 109.8 \\
\hline $\mathrm{C}(10)-\mathrm{C}(9)-\mathrm{H}(9 \mathrm{~B})$ & 109.8 \\
\hline $\mathrm{H}(9 \mathrm{~A})-\mathrm{C}(9)-\mathrm{H}(9 \mathrm{~B})$ & 108.3 \\
\hline $\mathrm{O}(1)-\mathrm{C}(10)-\mathrm{C}(9)$ & $110.82(19)$ \\
\hline $\mathrm{O}(1)-\mathrm{C}(10)-\mathrm{H}(10 \mathrm{~A})$ & 109.5 \\
\hline $\mathrm{C}(9)-\mathrm{C}(10)-\mathrm{H}(10 \mathrm{~A})$ & 109.5 \\
\hline $\mathrm{O}(1)-\mathrm{C}(10)-\mathrm{H}(10 \mathrm{~B})$ & 109.5 \\
\hline $\mathrm{C}(9)-\mathrm{C}(10)-\mathrm{H}(10 \mathrm{~B})$ & 109.5 \\
\hline $\mathrm{H}(10 \mathrm{~A})-\mathrm{C}(10)-\mathrm{H}(10 \mathrm{~B})$ & 108.1 \\
\hline $\mathrm{N}(2)-\mathrm{C}(11)-\mathrm{C}(12)$ & $109.40(19)$ \\
\hline $\mathrm{N}(2)-\mathrm{C}(11)-\mathrm{H}(11 \mathrm{~A})$ & 109.8 \\
\hline $\mathrm{C}(12)-\mathrm{C}(11)-\mathrm{H}(11 \mathrm{~A})$ & 109.8 \\
\hline $\mathrm{N}(2)-\mathrm{C}(11)-\mathrm{H}(11 \mathrm{~B})$ & 109.8 \\
\hline $\mathrm{C}(12)-\mathrm{C}(11)-\mathrm{H}(11 \mathrm{~B})$ & 109.8 \\
\hline $\mathrm{H}(11 \mathrm{~A})-\mathrm{C}(11)-\mathrm{H}(11 \mathrm{~B})$ & 108.2 \\
\hline $\mathrm{O}(1)-\mathrm{C}(12)-\mathrm{C}(11)$ & 111.96(19) \\
\hline $\mathrm{O}(1)-\mathrm{C}(12)-\mathrm{H}(12 \mathrm{~A})$ & 109.2 \\
\hline $\mathrm{C}(11)-\mathrm{C}(12)-\mathrm{H}(12 \mathrm{~A})$ & 109.2 \\
\hline $\mathrm{O}(1)-\mathrm{C}(12)-\mathrm{H}(12 \mathrm{~B})$ & 109.2 \\
\hline $\mathrm{C}(11)-\mathrm{C}(12)-\mathrm{H}(12 \mathrm{~B})$ & 109.2 \\
\hline $\mathrm{H}(12 \mathrm{~A})-\mathrm{C}(12)-\mathrm{H}(12 \mathrm{~B})$ & 107.9 \\
\hline
\end{tabular}

Symmetry transformations used to generate equivalent atoms: 
Table 4. Anisotropic displacement parameters $\left(\AA^{2} \mathrm{x} 10^{3}\right)$ for d1680_a. The anisotropic displacement factor exponent takes the form: $-2 \mathrm{p}^{2}\left[\mathrm{~h}^{2} \mathrm{a}^{* 2} \mathrm{U}^{11}+\ldots+2 \mathrm{hka} \mathrm{a}^{*} \mathrm{~b}^{*} \mathrm{U}^{12}\right]$

\begin{tabular}{|c|c|c|c|c|c|c|}
\hline & $\mathrm{U}^{11}$ & $\mathrm{U}^{22}$ & $\mathrm{U}^{33}$ & $\mathrm{U}^{23}$ & $\mathrm{U}^{13}$ & $\mathrm{U}^{12}$ \\
\hline $\mathrm{O}(1)$ & $26(1)$ & $36(1)$ & $18(1)$ & $-5(1)$ & $-1(1)$ & $-4(1)$ \\
\hline $\mathrm{N}(1)$ & $28(1)$ & $43(1)$ & $23(1)$ & $0(1)$ & $8(1)$ & $2(1)$ \\
\hline $\mathrm{N}(2)$ & $19(1)$ & $23(1)$ & $14(1)$ & $0(1)$ & $4(1)$ & $-3(1)$ \\
\hline $\mathrm{C}(1)$ & $20(1)$ & $40(1)$ & $34(2)$ & $-2(1)$ & $6(1)$ & $6(1)$ \\
\hline$C(2)$ & $22(1)$ & $35(2)$ & $24(1)$ & $0(1)$ & $-3(1)$ & $5(1)$ \\
\hline $\mathrm{C}(3)$ & $26(1)$ & $22(1)$ & $20(1)$ & 2(1) & 2(1) & 1(1) \\
\hline$C(4)$ & $18(1)$ & $18(1)$ & 19(1) & 1(1) & $5(1)$ & $-1(1)$ \\
\hline$C(5)$ & $20(1)$ & $18(1)$ & 19(1) & 1(1) & $3(1)$ & $-1(1)$ \\
\hline$C(6)$ & $23(1)$ & $34(1)$ & $22(1)$ & $-2(1)$ & $6(1)$ & $4(1)$ \\
\hline$C(7)$ & $25(1)$ & $24(1)$ & $12(1)$ & 1(1) & 1(1) & $-3(1)$ \\
\hline $\mathrm{C}(8)$ & $30(1)$ & $37(1)$ & $18(1)$ & $0(1)$ & $0(1)$ & $2(1)$ \\
\hline $\mathrm{C}(9)$ & $26(1)$ & $21(1)$ & $21(1)$ & $-2(1)$ & $6(1)$ & $-4(1)$ \\
\hline $\mathrm{C}(10)$ & $24(1)$ & $35(1)$ & $21(1)$ & $-4(1)$ & $5(1)$ & $-6(1)$ \\
\hline $\mathrm{C}(11)$ & $26(1)$ & $27(1)$ & $16(1)$ & $0(1)$ & $7(1)$ & $-1(1)$ \\
\hline$C(12)$ & 31(1) & $29(1)$ & $17(1)$ & $1(1)$ & $4(1)$ & $1(1)$ \\
\hline
\end{tabular}


Table 5. Hydrogen coordinates ( x 10 $)$ and isotropic displacement parameters $\left(\AA^{2} \times 10^{3}\right)$ for d1680_a.

\begin{tabular}{|c|c|c|c|c|}
\hline & $\mathrm{x}$ & $\mathrm{y}$ & $\mathrm{z}$ & $\mathrm{U}(\mathrm{eq})$ \\
\hline $\mathrm{H}(1 \mathrm{~A})$ & 176 & 4031 & 4529 & 38 \\
\hline $\mathrm{H}(2 \mathrm{~A})$ & 352 & 3883 & 1999 & 34 \\
\hline $\mathrm{H}(6 \mathrm{~A})$ & 2585 & 3997 & 6561 & 32 \\
\hline $\mathrm{H}(8 \mathrm{~A})$ & 1889 & 3590 & 159 & 44 \\
\hline $\mathrm{H}(8 \mathrm{~B})$ & 3559 & 3002 & 767 & 44 \\
\hline $\mathrm{H}(8 \mathrm{C})$ & 3719 & 4051 & 579 & 44 \\
\hline $\mathrm{H}(9 \mathrm{~A})$ & 6765 & 4991 & 6666 & 27 \\
\hline $\mathrm{H}(9 \mathrm{~B})$ & 7291 & 4418 & 5351 & 27 \\
\hline $\mathrm{H}(10 \mathrm{~A})$ & 9096 & 3534 & 7263 & 32 \\
\hline $\mathrm{H}(10 \mathrm{~B})$ & 9653 & 4555 & 7479 & 32 \\
\hline $\mathrm{H}(11 \mathrm{~A})$ & 4723 & 3231 & 8099 & 27 \\
\hline $\mathrm{H}(11 \mathrm{~B})$ & 5145 & 4269 & 8327 & 27 \\
\hline $\mathrm{H}(12 \mathrm{~A})$ & 7108 & 3458 & 10210 & 31 \\
\hline $\mathrm{H}(12 \mathrm{~B})$ & 7623 & 2880 & 8900 & 31 \\
\hline
\end{tabular}


Table 6. Torsion angles $\left[^{\circ}\right]$ for d1680_a.

\begin{tabular}{|c|c|}
\hline$C(6)-C(1)-C(2)-C(3)$ & $1.5(4)$ \\
\hline$C(1)-C(2)-C(3)-C(4)$ & $-0.1(4)$ \\
\hline$C(1)-C(2)-C(3)-C(8)$ & $178.3(2)$ \\
\hline$C(2)-C(3)-C(4)-C(5)$ & $-2.1(3)$ \\
\hline$C(8)-C(3)-C(4)-C(5)$ & $179.4(2)$ \\
\hline$C(2)-C(3)-C(4)-C(7)$ & $171.7(2)$ \\
\hline $\mathrm{C}(8)-\mathrm{C}(3)-\mathrm{C}(4)-\mathrm{C}(7)$ & $-6.8(3)$ \\
\hline $\mathrm{C}(9)-\mathrm{N}(2)-\mathrm{C}(5)-\mathrm{C}(6)$ & $-121.7(2)$ \\
\hline$C(11)-N(2)-C(5)-C(6)$ & $9.4(3)$ \\
\hline $\mathrm{C}(9)-\mathrm{N}(2)-\mathrm{C}(5)-\mathrm{C}(4)$ & $61.4(3)$ \\
\hline $\mathrm{C}(11)-\mathrm{N}(2)-\mathrm{C}(5)-\mathrm{C}(4)$ & $-167.53(19)$ \\
\hline$C(3)-C(4)-C(5)-C(6)$ & $2.9(3)$ \\
\hline$C(7)-C(4)-C(5)-C(6)$ & $-170.7(2)$ \\
\hline $\mathrm{C}(3)-\mathrm{C}(4)-\mathrm{C}(5)-\mathrm{N}(2)$ & $-180.0(2)$ \\
\hline $\mathrm{C}(7)-\mathrm{C}(4)-\mathrm{C}(5)-\mathrm{N}(2)$ & $6.4(3)$ \\
\hline$C(2)-C(1)-C(6)-C(5)$ & $-0.6(4)$ \\
\hline$N(2)-C(5)-C(6)-C(1)$ & $-178.5(2)$ \\
\hline $\mathrm{C}(4)-\mathrm{C}(5)-\mathrm{C}(6)-\mathrm{C}(1)$ & $-1.5(3)$ \\
\hline $\mathrm{C}(5)-\mathrm{N}(2)-\mathrm{C}(9)-\mathrm{C}(10)$ & $-167.97(18)$ \\
\hline $\mathrm{C}(11)-\mathrm{N}(2)-\mathrm{C}(9)-\mathrm{C}(10)$ & $57.9(2)$ \\
\hline $\mathrm{C}(12)-\mathrm{O}(1)-\mathrm{C}(10)-\mathrm{C}(9)$ & $60.7(2)$ \\
\hline
\end{tabular}


$\mathrm{N}(2)-\mathrm{C}(9)-\mathrm{C}(10)-\mathrm{O}(1)$

$\mathrm{C}(5)-\mathrm{N}(2)-\mathrm{C}(11)-\mathrm{C}(12)$

$\mathrm{C}(9)-\mathrm{N}(2)-\mathrm{C}(11)-\mathrm{C}(12)$

$\mathrm{C}(10)-\mathrm{O}(1)-\mathrm{C}(12)-\mathrm{C}(11)$

$\mathrm{N}(2)-\mathrm{C}(11)-\mathrm{C}(12)-\mathrm{O}(1)$
$-60.9(2)$

169.65(19)

$-56.1(2)$

$-59.3(2)$

$57.5(2)$

Symmetry transformations used to generate equivalent atoms: 


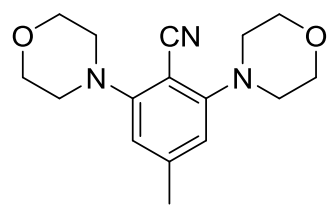

5b

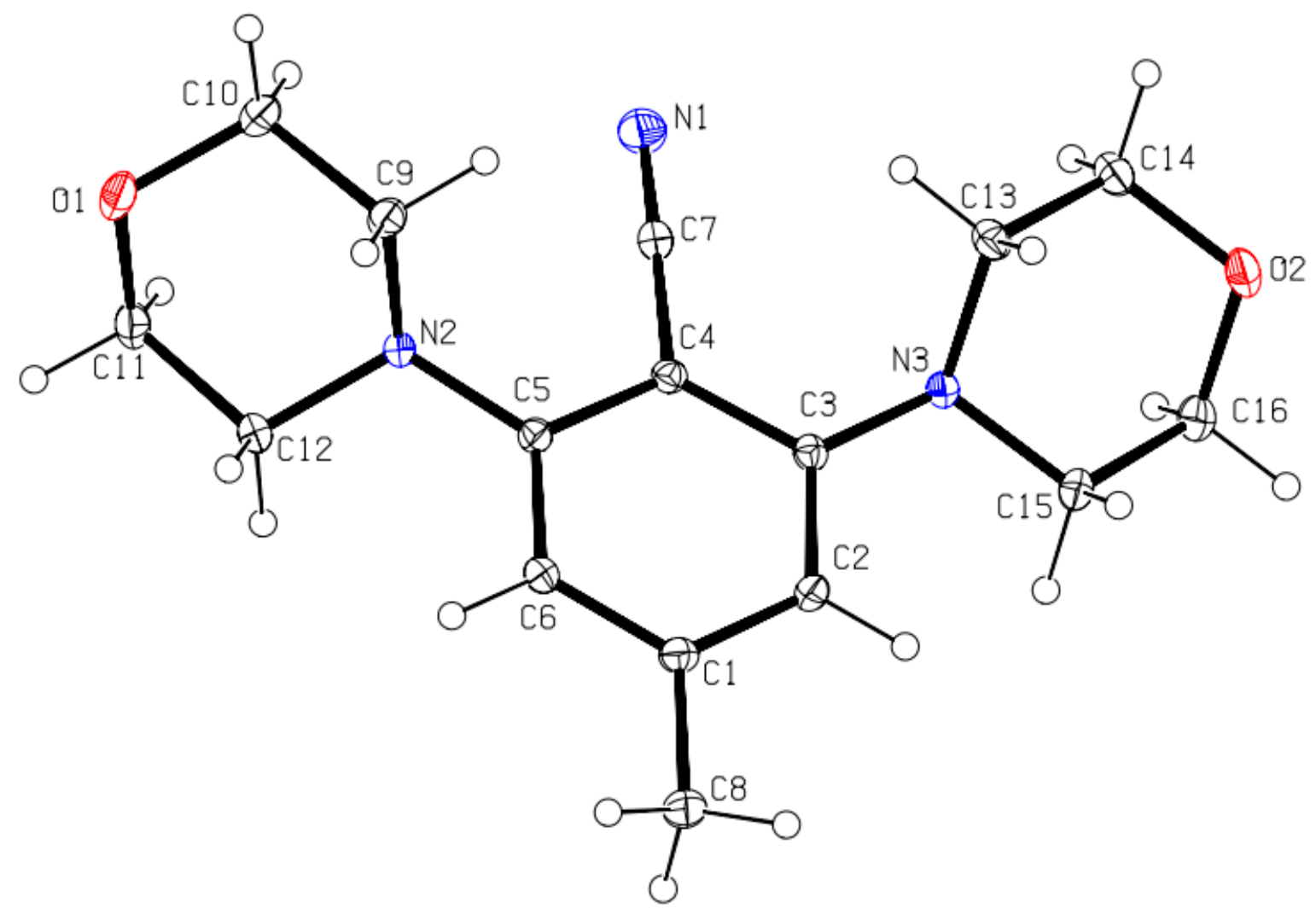


Table 1. Crystal data and structure refinement for $\mathrm{d} 1678$.

\begin{tabular}{|c|c|c|}
\hline Identification code & \multicolumn{2}{|l|}{ d1678 } \\
\hline Empirical formula & \multicolumn{2}{|l|}{$\mathrm{C} 16 \mathrm{H} 21 \mathrm{~N} 3 \mathrm{O} 2$} \\
\hline Formula weight & \multicolumn{2}{|l|}{287.36} \\
\hline Temperature & \multicolumn{2}{|l|}{$147(2) \mathrm{K}$} \\
\hline Wavelength & \multicolumn{2}{|l|}{$0.71073 \AA$} \\
\hline Crystal system & \multicolumn{2}{|l|}{ Triclinic } \\
\hline Space group & \multicolumn{2}{|l|}{$\mathrm{P}-1$} \\
\hline \multirow[t]{3}{*}{ Unit cell dimensions } & $a=9.1894(14) \AA$ & $\mathrm{a}=105.277(5)^{\circ}$. \\
\hline & $\mathrm{b}=9.4311(15) \AA$ & $\mathrm{b}=93.849(5)^{\circ}$ \\
\hline & $c=10.3177(15) \AA$ & $\mathrm{g}=116.710(4)^{\circ}$. \\
\hline Volume & \multicolumn{2}{|l|}{$752.5(2) \AA^{3}$} \\
\hline $\mathrm{Z}$ & \multicolumn{2}{|l|}{2} \\
\hline Density (calculated) & \multicolumn{2}{|l|}{$1.268 \mathrm{Mg} / \mathrm{m}^{3}$} \\
\hline Absorption coefficient & \multicolumn{2}{|l|}{$0.085 \mathrm{~mm}^{-1}$} \\
\hline $\mathrm{F}(000)$ & \multicolumn{2}{|l|}{308} \\
\hline Crystal size & \multicolumn{2}{|c|}{$0.370 \times 0.360 \times 0.100 \mathrm{~mm}^{3}$} \\
\hline Theta range for data collection & \multicolumn{2}{|l|}{2.095 to $27.523^{\circ}$. } \\
\hline Index ranges & \multicolumn{2}{|c|}{$-11<=\mathrm{h}<=11,-12<=\mathrm{k}<=12,-13<=\mathrm{l}<=13$} \\
\hline Reflections collected & \multicolumn{2}{|l|}{18570} \\
\hline Independent reflections & \multicolumn{2}{|c|}{$3464[\mathrm{R}(\mathrm{int})=0.0278]$} \\
\hline Completeness to theta $=25.242^{\circ}$ & \multicolumn{2}{|l|}{$100.0 \%$} \\
\hline Absorption correction & \multicolumn{2}{|c|}{ Semi-empirical from equivalents } \\
\hline
\end{tabular}


Max. and min. transmission

Refinement method

Data / restraints / parameters

Goodness-of-fit on $\mathrm{F}^{2}$

Final R indices [I $>2 \operatorname{sigma}(\mathrm{I})]$

$\mathrm{R}$ indices (all data)

Extinction coefficient

Largest diff. peak and hole
0.7456 and 0.7316

Full-matrix least-squares on $\mathrm{F}^{2}$

3464 / 0 / 191

1.038

$\mathrm{R} 1=0.0366, \mathrm{wR} 2=0.0901$

$\mathrm{R} 1=0.0484, \mathrm{wR} 2=0.0983$

$\mathrm{n} / \mathrm{a}$

0.251 and -0.222 e. $\AA^{-3}$

Table 2. Atomic coordinates $\left(\times 10^{4}\right)$ and equivalent isotropic displacement parameters $\left(\AA^{2} \times 10^{3}\right)$ for $\mathrm{d} 1678$. $U(\mathrm{eq})$ is defined as one third of the trace of the orthogonalized $\mathrm{U}^{\mathrm{ij}}$ tensor.

\begin{tabular}{|c|c|c|c|c|}
\hline & $\mathrm{x}$ & $\mathrm{y}$ & $\mathrm{z}$ & $\mathrm{U}(\mathrm{eq})$ \\
\hline $\mathrm{O}(1)$ & $8769(1)$ & $2329(1)$ & $11918(1)$ & $29(1)$ \\
\hline $\mathrm{O}(2)$ & $3992(1)$ & $-3957(1)$ & $1840(1)$ & $28(1)$ \\
\hline $\mathrm{N}(1)$ & $5857(1)$ & $-2480(1)$ & $7344(1)$ & $30(1)$ \\
\hline $\mathrm{N}(2)$ & $8831(1)$ & $1568(1)$ & $9087(1)$ & $18(1)$ \\
\hline $\mathrm{N}(3)$ & $6568(1)$ & $-1528(1)$ & $4207(1)$ & $18(1)$ \\
\hline$C(1)$ & $10109(1)$ & $2812(1)$ & $6017(1)$ & $20(1)$ \\
\hline$C(2)$ & $8930(1)$ & 1359(1) & $4969(1)$ & 19(1) \\
\hline$C(3)$ & $7737(1)$ & $-27(1)$ & $5254(1)$ & $16(1)$ \\
\hline $\mathrm{C}(4)$ & $7767(1)$ & $79(1)$ & $6649(1)$ & $17(1)$ \\
\hline$C(5)$ & $8913(1)$ & $1569(1)$ & $7721(1)$ & $17(1)$ \\
\hline
\end{tabular}




\begin{tabular}{|c|c|c|c|c|}
\hline $\mathrm{C}(6)$ & 10075(1) & 2921(1) & 7391(1) & 19(1) \\
\hline $\mathrm{C}(7)$ & $6702(1)$ & $-1350(1)$ & $7025(1)$ & $19(1)$ \\
\hline $\mathrm{C}(8)$ & 11404(2) & $4256(2)$ & $5660(1)$ & $27(1)$ \\
\hline $\mathrm{C}(9)$ & $7473(2)$ & $1812(2)$ & 9583(1) & $23(1)$ \\
\hline $\mathrm{C}(10)$ & $7257(2)$ & $1363(2)$ & 10886(1) & $30(1)$ \\
\hline $\mathrm{C}(11)$ & $10085(2)$ & 2112(2) & 11411(1) & $25(1)$ \\
\hline $\mathrm{C}(12)$ & $10378(1)$ & $2635(2)$ & 10141(1) & $23(1)$ \\
\hline $\mathrm{C}(13)$ & $4793(1)$ & $-2041(2)$ & $4182(1)$ & 21(1) \\
\hline $\mathrm{C}(14)$ & $3710(2)$ & $-3820(2)$ & $3200(1)$ & $25(1)$ \\
\hline $\mathrm{C}(15)$ & $6838(2)$ & $-1672(2)$ & $2807(1)$ & $23(1$ \\
\hline $\mathrm{C}(16)$ & $5708(2)$ & $-3466(2)$ & $1858(1)$ & $28(1)$ \\
\hline
\end{tabular}

Table 3. Bond lengths $\left[\AA ̊ \cap\right.$ and angles $\left[{ }^{\circ}\right]$ for $\mathrm{d} 1678$.

\begin{tabular}{ll}
\hline $\mathrm{O}(1)-\mathrm{C}(11)$ & $1.4244(16)$ \\
$\mathrm{O}(1)-\mathrm{C}(10)$ & $1.4308(16)$ \\
$\mathrm{O}(2)-\mathrm{C}(14)$ & $1.4270(15)$ \\
$\mathrm{O}(2)-\mathrm{C}(16)$ & $1.1491(16)$ \\
$\mathrm{N}(1)-\mathrm{C}(7)$ & $1.4177(14)$ \\
$\mathrm{N}(2)-\mathrm{C}(5)$ & $1.4649(14)$ \\
$\mathrm{N}(2)-\mathrm{C}(12)$ & $1.4682(15)$ \\
$\mathrm{N}(2)-\mathrm{C}(9)$ &
\end{tabular}




\begin{tabular}{|c|c|}
\hline $\mathrm{N}(3)-\mathrm{C}(3)$ & $1.4131(14)$ \\
\hline$N(3)-C(15)$ & $1.4637(14)$ \\
\hline $\mathrm{N}(3)-\mathrm{C}(13)$ & $1.4747(14)$ \\
\hline $\mathrm{C}(1)-\mathrm{C}(2)$ & $1.3960(16)$ \\
\hline$C(1)-C(6)$ & $1.3978(16)$ \\
\hline $\mathrm{C}(1)-\mathrm{C}(8)$ & $1.5075(16)$ \\
\hline $\mathrm{C}(2)-\mathrm{C}(3)$ & $1.3979(16)$ \\
\hline $\mathrm{C}(2)-\mathrm{H}(2 \mathrm{~A})$ & 0.9500 \\
\hline$C(3)-C(4)$ & $1.4135(15)$ \\
\hline$C(4)-C(5)$ & $1.4145(15)$ \\
\hline$C(4)-C(7)$ & $1.4380(16)$ \\
\hline$C(5)-C(6)$ & $1.3905(16)$ \\
\hline $\mathrm{C}(6)-\mathrm{H}(6 \mathrm{~A})$ & 0.9500 \\
\hline $\mathrm{C}(8)-\mathrm{H}(8 \mathrm{~A})$ & 0.9800 \\
\hline $\mathrm{C}(8)-\mathrm{H}(8 \mathrm{~B})$ & 0.9800 \\
\hline $\mathrm{C}(8)-\mathrm{H}(8 \mathrm{C})$ & 0.9800 \\
\hline $\mathrm{C}(9)-\mathrm{C}(10)$ & $1.5122(16)$ \\
\hline $\mathrm{C}(9)-\mathrm{H}(9 \mathrm{~A})$ & 0.9900 \\
\hline $\mathrm{C}(9)-\mathrm{H}(9 \mathrm{~B})$ & 0.9900 \\
\hline $\mathrm{C}(10)-\mathrm{H}(10 \mathrm{~A})$ & 0.9900 \\
\hline $\mathrm{C}(10)-\mathrm{H}(10 \mathrm{~B})$ & 0.9900 \\
\hline $\mathrm{C}(11)-\mathrm{C}(12)$ & $1.5167(16)$ \\
\hline $\mathrm{C}(11)-\mathrm{H}(11 \mathrm{~A})$ & 0.9900 \\
\hline
\end{tabular}




\begin{tabular}{|c|c|}
\hline $\mathrm{C}(11)-\mathrm{H}(11 \mathrm{~B})$ & 0.9900 \\
\hline $\mathrm{C}(12)-\mathrm{H}(12 \mathrm{~A})$ & 0.9900 \\
\hline $\mathrm{C}(12)-\mathrm{H}(12 \mathrm{~B})$ & 0.9900 \\
\hline $\mathrm{C}(13)-\mathrm{C}(14)$ & $1.5161(16)$ \\
\hline $\mathrm{C}(13)-\mathrm{H}(13 \mathrm{~A})$ & 0.9900 \\
\hline $\mathrm{C}(13)-\mathrm{H}(13 \mathrm{~B})$ & 0.9900 \\
\hline $\mathrm{C}(14)-\mathrm{H}(14 \mathrm{~A})$ & 0.9900 \\
\hline $\mathrm{C}(14)-\mathrm{H}(14 \mathrm{~B})$ & 0.9900 \\
\hline$C(15)-C(16)$ & $1.5197(17)$ \\
\hline $\mathrm{C}(15)-\mathrm{H}(15 \mathrm{~A})$ & 0.9900 \\
\hline $\mathrm{C}(15)-\mathrm{H}(15 \mathrm{~B})$ & 0.9900 \\
\hline$C(16)-H(16 A)$ & 0.9900 \\
\hline $\mathrm{C}(16)-\mathrm{H}(16 \mathrm{~B})$ & 0.9900 \\
\hline $\mathrm{C}(11)-\mathrm{O}(1)-\mathrm{C}(10)$ & $110.52(9)$ \\
\hline $\mathrm{C}(14)-\mathrm{O}(2)-\mathrm{C}(16)$ & $109.51(9)$ \\
\hline $\mathrm{C}(5)-\mathrm{N}(2)-\mathrm{C}(12)$ & $117.45(9)$ \\
\hline $\mathrm{C}(5)-\mathrm{N}(2)-\mathrm{C}(9)$ & $114.31(9)$ \\
\hline $\mathrm{C}(12)-\mathrm{N}(2)-\mathrm{C}(9)$ & $109.80(9)$ \\
\hline $\mathrm{C}(3)-\mathrm{N}(3)-\mathrm{C}(15)$ & $116.25(9)$ \\
\hline $\mathrm{C}(3)-\mathrm{N}(3)-\mathrm{C}(13)$ & $116.38(9)$ \\
\hline $\mathrm{C}(15)-\mathrm{N}(3)-\mathrm{C}(13)$ & $108.63(9)$ \\
\hline$C(2)-C(1)-C(6)$ & $119.71(10)$ \\
\hline
\end{tabular}




\begin{tabular}{|c|c|}
\hline $\mathrm{C}(2)-\mathrm{C}(1)-\mathrm{C}(8)$ & $119.82(10)$ \\
\hline $\mathrm{C}(6)-\mathrm{C}(1)-\mathrm{C}(8)$ & $120.47(10)$ \\
\hline $\mathrm{C}(1)-\mathrm{C}(2)-\mathrm{C}(3)$ & $121.66(10)$ \\
\hline $\mathrm{C}(1)-\mathrm{C}(2)-\mathrm{H}(2 \mathrm{~A})$ & 119.2 \\
\hline $\mathrm{C}(3)-\mathrm{C}(2)-\mathrm{H}(2 \mathrm{~A})$ & 119.2 \\
\hline $\mathrm{C}(2)-\mathrm{C}(3)-\mathrm{N}(3)$ & $122.50(10)$ \\
\hline $\mathrm{C}(2)-\mathrm{C}(3)-\mathrm{C}(4)$ & $117.79(10)$ \\
\hline $\mathrm{N}(3)-\mathrm{C}(3)-\mathrm{C}(4)$ & $119.66(10)$ \\
\hline$C(3)-C(4)-C(5)$ & $121.02(10)$ \\
\hline$C(3)-C(4)-C(7)$ & $121.19(10)$ \\
\hline$C(5)-C(4)-C(7)$ & $117.70(10)$ \\
\hline$C(6)-C(5)-C(4)$ & $119.24(10)$ \\
\hline $\mathrm{C}(6)-\mathrm{C}(5)-\mathrm{N}(2)$ & $123.73(10)$ \\
\hline $\mathrm{C}(4)-\mathrm{C}(5)-\mathrm{N}(2)$ & $117.00(10)$ \\
\hline$C(5)-C(6)-C(1)$ & $120.47(10)$ \\
\hline $\mathrm{C}(5)-\mathrm{C}(6)-\mathrm{H}(6 \mathrm{~A})$ & 119.8 \\
\hline $\mathrm{C}(1)-\mathrm{C}(6)-\mathrm{H}(6 \mathrm{~A})$ & 119.8 \\
\hline $\mathrm{N}(1)-\mathrm{C}(7)-\mathrm{C}(4)$ & $179.08(12)$ \\
\hline $\mathrm{C}(1)-\mathrm{C}(8)-\mathrm{H}(8 \mathrm{~A})$ & 109.5 \\
\hline $\mathrm{C}(1)-\mathrm{C}(8)-\mathrm{H}(8 \mathrm{~B})$ & 109.5 \\
\hline $\mathrm{H}(8 \mathrm{~A})-\mathrm{C}(8)-\mathrm{H}(8 \mathrm{~B})$ & 109.5 \\
\hline $\mathrm{C}(1)-\mathrm{C}(8)-\mathrm{H}(8 \mathrm{C})$ & 109.5 \\
\hline $\mathrm{H}(8 \mathrm{~A})-\mathrm{C}(8)-\mathrm{H}(8 \mathrm{C})$ & 109.5 \\
\hline
\end{tabular}




\begin{tabular}{|c|c|}
\hline $\mathrm{H}(8 \mathrm{~B})-\mathrm{C}(8)-\mathrm{H}(8 \mathrm{C})$ & 109.5 \\
\hline $\mathrm{N}(2)-\mathrm{C}(9)-\mathrm{C}(10)$ & $108.76(10)$ \\
\hline $\mathrm{N}(2)-\mathrm{C}(9)-\mathrm{H}(9 \mathrm{~A})$ & 109.9 \\
\hline $\mathrm{C}(10)-\mathrm{C}(9)-\mathrm{H}(9 \mathrm{~A})$ & 109.9 \\
\hline $\mathrm{N}(2)-\mathrm{C}(9)-\mathrm{H}(9 \mathrm{~B})$ & 109.9 \\
\hline $\mathrm{C}(10)-\mathrm{C}(9)-\mathrm{H}(9 \mathrm{~B})$ & 109.9 \\
\hline $\mathrm{H}(9 \mathrm{~A})-\mathrm{C}(9)-\mathrm{H}(9 \mathrm{~B})$ & 108.3 \\
\hline $\mathrm{O}(1)-\mathrm{C}(10)-\mathrm{C}(9)$ & $111.73(10)$ \\
\hline $\mathrm{O}(1)-\mathrm{C}(10)-\mathrm{H}(10 \mathrm{~A})$ & 109.3 \\
\hline $\mathrm{C}(9)-\mathrm{C}(10)-\mathrm{H}(10 \mathrm{~A})$ & 109.3 \\
\hline $\mathrm{O}(1)-\mathrm{C}(10)-\mathrm{H}(10 \mathrm{~B})$ & 109.3 \\
\hline C(9)-C(10)-H(10B) & 109.3 \\
\hline $\mathrm{H}(10 \mathrm{~A})-\mathrm{C}(10)-\mathrm{H}(10 \mathrm{~B})$ & 107.9 \\
\hline $\mathrm{O}(1)-\mathrm{C}(11)-\mathrm{C}(12)$ & $111.16(10)$ \\
\hline $\mathrm{O}(1)-\mathrm{C}(11)-\mathrm{H}(11 \mathrm{~A})$ & 109.4 \\
\hline $\mathrm{C}(12)-\mathrm{C}(11)-\mathrm{H}(11 \mathrm{~A})$ & 109.4 \\
\hline $\mathrm{O}(1)-\mathrm{C}(11)-\mathrm{H}(11 \mathrm{~B})$ & 109.4 \\
\hline $\mathrm{C}(12)-\mathrm{C}(11)-\mathrm{H}(11 \mathrm{~B})$ & 109.4 \\
\hline $\mathrm{H}(11 \mathrm{~A})-\mathrm{C}(11)-\mathrm{H}(11 \mathrm{~B})$ & 108.0 \\
\hline $\mathrm{N}(2)-\mathrm{C}(12)-\mathrm{C}(11)$ & $107.57(9)$ \\
\hline $\mathrm{N}(2)-\mathrm{C}(12)-\mathrm{H}(12 \mathrm{~A})$ & 110.2 \\
\hline $\mathrm{C}(11)-\mathrm{C}(12)-\mathrm{H}(12 \mathrm{~A})$ & 110.2 \\
\hline $\mathrm{N}(2)-\mathrm{C}(12)-\mathrm{H}(12 \mathrm{~B})$ & 110.2 \\
\hline
\end{tabular}




\begin{tabular}{|c|c|}
\hline $\mathrm{C}(11)-\mathrm{C}(12)-\mathrm{H}(12 \mathrm{~B})$ & 110.2 \\
\hline $\mathrm{H}(12 \mathrm{~A})-\mathrm{C}(12)-\mathrm{H}(12 \mathrm{~B})$ & 108.5 \\
\hline $\mathrm{N}(3)-\mathrm{C}(13)-\mathrm{C}(14)$ & $109.88(9)$ \\
\hline $\mathrm{N}(3)-\mathrm{C}(13)-\mathrm{H}(13 \mathrm{~A})$ & 109.7 \\
\hline $\mathrm{C}(14)-\mathrm{C}(13)-\mathrm{H}(13 \mathrm{~A})$ & 109.7 \\
\hline $\mathrm{N}(3)-\mathrm{C}(13)-\mathrm{H}(13 \mathrm{~B})$ & 109.7 \\
\hline $\mathrm{C}(14)-\mathrm{C}(13)-\mathrm{H}(13 \mathrm{~B})$ & 109.7 \\
\hline $\mathrm{H}(13 \mathrm{~A})-\mathrm{C}(13)-\mathrm{H}(13 \mathrm{~B})$ & 108.2 \\
\hline $\mathrm{O}(2)-\mathrm{C}(14)-\mathrm{C}(13)$ & $111.35(10)$ \\
\hline $\mathrm{O}(2)-\mathrm{C}(14)-\mathrm{H}(14 \mathrm{~A})$ & 109.4 \\
\hline $\mathrm{C}(13)-\mathrm{C}(14)-\mathrm{H}(14 \mathrm{~A})$ & 109.4 \\
\hline $\mathrm{O}(2)-\mathrm{C}(14)-\mathrm{H}(14 \mathrm{~B})$ & 109.4 \\
\hline $\mathrm{C}(13)-\mathrm{C}(14)-\mathrm{H}(14 \mathrm{~B})$ & 109.4 \\
\hline $\mathrm{H}(14 \mathrm{~A})-\mathrm{C}(14)-\mathrm{H}(14 \mathrm{~B})$ & 108.0 \\
\hline $\mathrm{N}(3)-\mathrm{C}(15)-\mathrm{C}(16)$ & $109.68(10)$ \\
\hline $\mathrm{N}(3)-\mathrm{C}(15)-\mathrm{H}(15 \mathrm{~A})$ & 109.7 \\
\hline$C(16)-C(15)-H(15 A)$ & 109.7 \\
\hline $\mathrm{N}(3)-\mathrm{C}(15)-\mathrm{H}(15 \mathrm{~B})$ & 109.7 \\
\hline $\mathrm{C}(16)-\mathrm{C}(15)-\mathrm{H}(15 \mathrm{~B})$ & 109.7 \\
\hline $\mathrm{H}(15 \mathrm{~A})-\mathrm{C}(15)-\mathrm{H}(15 \mathrm{~B})$ & 108.2 \\
\hline $\mathrm{O}(2)-\mathrm{C}(16)-\mathrm{C}(15)$ & $111.40(10)$ \\
\hline $\mathrm{O}(2)-\mathrm{C}(16)-\mathrm{H}(16 \mathrm{~A})$ & 109.3 \\
\hline$C(15)-C(16)-H(16 A)$ & 109.3 \\
\hline
\end{tabular}


$\mathrm{O}(2)-\mathrm{C}(16)-\mathrm{H}(16 \mathrm{~B})$

$\mathrm{C}(15)-\mathrm{C}(16)-\mathrm{H}(16 \mathrm{~B})$

$\mathrm{H}(16 \mathrm{~A})-\mathrm{C}(16)-\mathrm{H}(16 \mathrm{~B})$
109.3

109.3

108.0

Symmetry transformations used to generate equivalent atoms:

Table 4. Anisotropic displacement parameters $\left(\AA^{2} \times 10^{3}\right)$ for d1678. The anisotropic displacement factor exponent takes the form: $-2 p^{2}\left[h^{2} a^{* 2} U^{11}+\ldots+2 h k a^{*} b^{*} U^{12}\right]$

\begin{tabular}{|c|c|c|c|c|c|c|}
\hline & $\mathrm{U}^{11}$ & $\mathrm{U}^{22}$ & $\mathrm{U}^{33}$ & $\mathrm{U}^{23}$ & $\mathrm{U}^{13}$ & $\mathrm{U}^{12}$ \\
\hline $\mathrm{O}(1)$ & $34(1)$ & $38(1)$ & $15(1)$ & $7(1)$ & $6(1)$ & $18(1)$ \\
\hline $\mathrm{O}(2)$ & $25(1)$ & $28(1)$ & $20(1)$ & $2(1)$ & $-3(1)$ & $9(1)$ \\
\hline $\mathrm{N}(1)$ & $30(1)$ & $25(1)$ & $32(1)$ & $13(1)$ & $6(1)$ & $8(1)$ \\
\hline $\mathrm{N}(2)$ & $15(1)$ & $21(1)$ & $14(1)$ & $5(1)$ & $2(1)$ & $6(1)$ \\
\hline $\mathrm{N}(3)$ & $16(1)$ & $18(1)$ & $15(1)$ & $4(1)$ & $2(1)$ & $7(1)$ \\
\hline$C(1)$ & $18(1)$ & $20(1)$ & 21(1) & $9(1)$ & $6(1)$ & $9(1)$ \\
\hline$C(2)$ & $20(1)$ & $22(1)$ & $15(1)$ & $8(1)$ & $5(1)$ & $10(1)$ \\
\hline$C(3)$ & $16(1)$ & $18(1)$ & $16(1)$ & $5(1)$ & $2(1)$ & $10(1)$ \\
\hline$C(4)$ & $15(1)$ & $18(1)$ & $18(1)$ & $7(1)$ & $4(1)$ & $8(1)$ \\
\hline$C(5)$ & $16(1)$ & $20(1)$ & $16(1)$ & $6(1)$ & $3(1)$ & $9(1)$ \\
\hline$C(6)$ & $17(1)$ & $18(1)$ & $17(1)$ & $4(1)$ & $2(1)$ & $5(1)$ \\
\hline$C(7)$ & $20(1)$ & $20(1)$ & $17(1)$ & $4(1)$ & 1(1) & $9(1)$ \\
\hline $\mathrm{C}(8)$ & $25(1)$ & $24(1)$ & $25(1)$ & $11(1)$ & $7(1)$ & $6(1)$ \\
\hline
\end{tabular}




\begin{tabular}{lcccccc}
$\mathrm{C}(9)$ & $22(1)$ & $30(1)$ & $19(1)$ & $10(1)$ & $6(1)$ & $14(1)$ \\
$\mathrm{C}(10)$ & $27(1)$ & $43(1)$ & $21(1)$ & $14(1)$ & $9(1)$ & $15(1)$ \\
$\mathrm{C}(11)$ & $26(1)$ & $26(1)$ & $18(1)$ & $6(1)$ & $-1(1)$ & $11(1)$ \\
$\mathrm{C}(12)$ & $18(1)$ & $26(1)$ & $17(1)$ & $5(1)$ & $0(1)$ & $6(1)$ \\
$\mathrm{C}(13)$ & $17(1)$ & $22(1)$ & $22(1)$ & $4(1)$ & $2(1)$ & $10(1)$ \\
$\mathrm{C}(14)$ & $20(1)$ & $23(1)$ & $24(1)$ & $4(1)$ & $0(1)$ & $8(1)$ \\
$\mathrm{C}(15)$ & $24(1)$ & $24(1)$ & $16(1)$ & $5(1)$ & $3(1)$ & $9(1)$ \\
$\mathrm{C}(16)$ & $27(1)$ & $28(1)$ & $19(1)$ & $1(1)$ & $3(1)$ & $11(1)$ \\
& & & & & \\
\hline
\end{tabular}

Table 5. Hydrogen coordinates ( $\left.\times 10^{4}\right)$ and isotropic displacement parameters $\left(\AA^{2} \times 10^{3}\right.$ ) for d 1678 .

$\begin{array}{llll}\text { x } & \text { y } & \text { U } & \end{array}$

$\begin{array}{lcccc}\mathrm{H}(2 \mathrm{~A}) & 8939 & 1310 & 4038 & 23 \\ \mathrm{H}(6 \mathrm{~A}) & 10851 & 3925 & 8105 & 23 \\ \mathrm{H}(8 \mathrm{~A}) & 10955 & 4260 & 4773 & 40 \\ \mathrm{H}(8 \mathrm{~B}) & 12402 & 4129 & 5600 & 40 \\ \mathrm{H}(8 \mathrm{C}) & 11699 & 5317 & 6375 & 40 \\ \mathrm{H}(9 \mathrm{~A}) & 6424 & 1089 & 8874 & 27 \\ \mathrm{H}(9 \mathrm{~B}) & 7742 & 2998 & 9771 & 27 \\ \mathrm{H}(10 \mathrm{~A}) & 6370 & 1564 & 11246 & 36\end{array}$




\begin{tabular}{lcccc}
$\mathrm{H}(10 \mathrm{~B})$ & 6899 & 152 & 10671 & 36 \\
$\mathrm{H}(11 \mathrm{~A})$ & 9800 & 917 & 11181 & 30 \\
$\mathrm{H}(11 \mathrm{~B})$ & 11122 & 2794 & 12135 & 30 \\
$\mathrm{H}(12 \mathrm{~A})$ & 10676 & 3832 & 10360 & 28 \\
$\mathrm{H}(12 \mathrm{~B})$ & 11303 & 2489 & 9807 & 28 \\
$\mathrm{H}(13 \mathrm{~A})$ & 4509 & -1264 & 3884 & 25 \\
$\mathrm{H}(13 \mathrm{~B})$ & 4582 & -1984 & 5118 & 30 \\
$\mathrm{H}(14 \mathrm{~A})$ & 3959 & -4599 & 3529 & 30 \\
$\mathrm{H}(14 \mathrm{~B})$ & 2522 & -4152 & 3187 & 27 \\
$\mathrm{H}(15 \mathrm{~A})$ & 8018 & -1364 & 2800 & 33 \\
$\mathrm{H}(15 \mathrm{~B})$ & 6592 & -890 & 2479 & 27 \\
$\mathrm{H}(16 \mathrm{~A})$ & 5900 & -3558 & 914 & 33 \\
$\mathrm{H}(16 \mathrm{~B})$ & 5989 & -4237 & 2169 & \\
\hline
\end{tabular}

Table 6. Torsion angles $\left[{ }^{\circ}\right]$ for $\mathrm{d} 1678$.

$\begin{array}{lr}\mathrm{C}(6)-\mathrm{C}(1)-\mathrm{C}(2)-\mathrm{C}(3) & -2.08(17) \\ \mathrm{C}(8)-\mathrm{C}(1)-\mathrm{C}(2)-\mathrm{C}(3) & 178.18(11) \\ \mathrm{C}(1)-\mathrm{C}(2)-\mathrm{C}(3)-\mathrm{N}(3) & -177.93(10) \\ \mathrm{C}(1)-\mathrm{C}(2)-\mathrm{C}(3)-\mathrm{C}(4) & -0.60(16) \\ \mathrm{C}(15)-\mathrm{N}(3)-\mathrm{C}(3)-\mathrm{C}(2) & 9.76(15) \\ \mathrm{C}(13)-\mathrm{N}(3)-\mathrm{C}(3)-\mathrm{C}(2) & -120.18(12) \\ \mathrm{C}(15)-\mathrm{N}(3)-\mathrm{C}(3)-\mathrm{C}(4) & -167.52(10)\end{array}$




\begin{tabular}{|c|c|}
\hline $\mathrm{C}(13)-\mathrm{N}(3)-\mathrm{C}(3)-\mathrm{C}(4)$ & $62.53(13)$ \\
\hline $\mathrm{C}(2)-\mathrm{C}(3)-\mathrm{C}(4)-\mathrm{C}(5)$ & $3.12(16)$ \\
\hline $\mathrm{N}(3)-\mathrm{C}(3)-\mathrm{C}(4)-\mathrm{C}(5)$ & $-179.47(10)$ \\
\hline $\mathrm{C}(2)-\mathrm{C}(3)-\mathrm{C}(4)-\mathrm{C}(7)$ & $-173.33(10)$ \\
\hline $\mathrm{N}(3)-\mathrm{C}(3)-\mathrm{C}(4)-\mathrm{C}(7)$ & $4.08(15)$ \\
\hline $\mathrm{C}(3)-\mathrm{C}(4)-\mathrm{C}(5)-\mathrm{C}(6)$ & $-2.95(16)$ \\
\hline$C(7)-C(4)-C(5)-C(6)$ & $173.62(10)$ \\
\hline $\mathrm{C}(3)-\mathrm{C}(4)-\mathrm{C}(5)-\mathrm{N}(2)$ & 178.94(9) \\
\hline $\mathrm{C}(7)-\mathrm{C}(4)-\mathrm{C}(5)-\mathrm{N}(2)$ & $-4.49(15)$ \\
\hline $\mathrm{C}(12)-\mathrm{N}(2)-\mathrm{C}(5)-\mathrm{C}(6)$ & $-27.43(16)$ \\
\hline $\mathrm{C}(9)-\mathrm{N}(2)-\mathrm{C}(5)-\mathrm{C}(6)$ & $103.37(13)$ \\
\hline $\mathrm{C}(12)-\mathrm{N}(2)-\mathrm{C}(5)-\mathrm{C}(4)$ & $150.59(10)$ \\
\hline $\mathrm{C}(9)-\mathrm{N}(2)-\mathrm{C}(5)-\mathrm{C}(4)$ & $-78.61(12)$ \\
\hline $\mathrm{C}(4)-\mathrm{C}(5)-\mathrm{C}(6)-\mathrm{C}(1)$ & $0.20(16)$ \\
\hline $\mathrm{N}(2)-\mathrm{C}(5)-\mathrm{C}(6)-\mathrm{C}(1)$ & $178.17(10)$ \\
\hline $\mathrm{C}(2)-\mathrm{C}(1)-\mathrm{C}(6)-\mathrm{C}(5)$ & $2.28(17)$ \\
\hline $\mathrm{C}(8)-\mathrm{C}(1)-\mathrm{C}(6)-\mathrm{C}(5)$ & $-177.98(11)$ \\
\hline $\mathrm{C}(5)-\mathrm{N}(2)-\mathrm{C}(9)-\mathrm{C}(10)$ & $166.22(10)$ \\
\hline $\mathrm{C}(12)-\mathrm{N}(2)-\mathrm{C}(9)-\mathrm{C}(10)$ & $-59.34(13)$ \\
\hline $\mathrm{C}(11)-\mathrm{O}(1)-\mathrm{C}(10)-\mathrm{C}(9)$ & $-56.83(14)$ \\
\hline $\mathrm{N}(2)-\mathrm{C}(9)-\mathrm{C}(10)-\mathrm{O}(1)$ & $56.92(14)$ \\
\hline $\mathrm{C}(10)-\mathrm{O}(1)-\mathrm{C}(11)-\mathrm{C}(12)$ & $58.67(13)$ \\
\hline $\mathrm{C}(5)-\mathrm{N}(2)-\mathrm{C}(12)-\mathrm{C}(11)$ & $-166.43(10)$ \\
\hline
\end{tabular}




\begin{tabular}{lc}
$\mathrm{C}(9)-\mathrm{N}(2)-\mathrm{C}(12)-\mathrm{C}(11)$ & $60.73(12)$ \\
$\mathrm{O}(1)-\mathrm{C}(11)-\mathrm{C}(12)-\mathrm{N}(2)$ & $-60.48(13)$ \\
$\mathrm{C}(3)-\mathrm{N}(3)-\mathrm{C}(13)-\mathrm{C}(14)$ & $-168.86(9)$ \\
$\mathrm{C}(15)-\mathrm{N}(3)-\mathrm{C}(13)-\mathrm{C}(14)$ & $57.66(12)$ \\
$\mathrm{C}(16)-\mathrm{O}(2)-\mathrm{C}(14)-\mathrm{C}(13)$ & $58.56(13)$ \\
$\mathrm{N}(3)-\mathrm{C}(13)-\mathrm{C}(14)-\mathrm{O}(2)$ & $-58.79(12)$ \\
$\mathrm{C}(3)-\mathrm{N}(3)-\mathrm{C}(15)-\mathrm{C}(16)$ & $168.70(10)$ \\
$\mathrm{C}(13)-\mathrm{N}(3)-\mathrm{C}(15)-\mathrm{C}(16)$ & \\
$\mathrm{C}(14)-\mathrm{O}(2)-\mathrm{C}(16)-\mathrm{C}(15)$ & $-57.75(12)$ \\
$\mathrm{N}(3)-\mathrm{C}(15)-\mathrm{C}(16)-\mathrm{O}(2)$ & $-58.81(13)$ \\
\hline
\end{tabular}

Symmetry transformations used to generate equivalent atoms: 